\title{
Numerische Untersuchungen zum optischen Durchbruch von Femtosekunden-Laserpulsen in Wasser
}

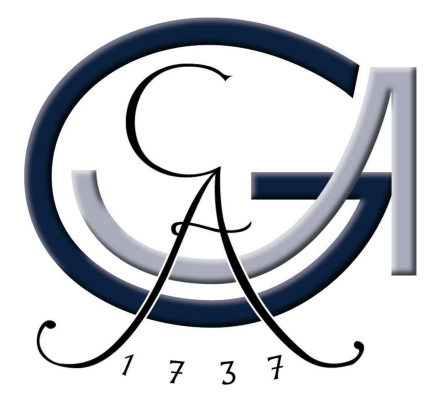

Dissertation zur Erlangung des Doktorgrades der Mathematisch-Naturwissenschaftlichen Fakultäten der Georg-August-Universität zu Göttingen

\author{
Vorgelegt von \\ Karsten Köhler \\ aus \\ Göttingen
}

Göttingen, 2010 
D7

Referent: Prof. Dr. W. Lauterborn

Korreferent: Prof. Dr. U. Parlitz

Tag der mündlichen Prüfung: 13.10.2010 


\section{Inhaltsverzeichnis}

Einleitung 4

1 Theoretische Grundlagen der Pulsausbreitung 6

1.1 Maxwell-Gleichungen . . . . . . . . . . . . . . . . . 6

1.2 Lineare Polarisation . . . . . . . . . . . . . . . . . . 7

1.3 Nichtlineare Polarisation: Kerr-Effekt . . . . . . . . . . . . . 10

1.4 Wellengleichung . . . . . . . . . . . . . . . . . . 11

1.5 Lösungsmethoden auf Grundlage von komplexen Einhüllenden . 12

1.5.1 Die komplexe Einhüllende eines ultrakurzen Laserpulses. 12

1.5.2 Herleitung der Einhüllendengleichung . . . . . . . . . . . 15

2 Berechnung der Pulsausbreitung 20

2.1 Split-Operator-Verfahren . . . . . . . . . . . . . . 20

2.2 Berechnung der auftretenden Terme . . . . . . . . . . . . . 22

2.3 Schrittweitensteuerung und Diskretisierung . . . . . . . . . . . 22

2.4 Beispiele . . . . . . . . . . . . . . . . . . . . . . . . 24

3 Pulsausbreitung in räumlich ausgedehnten Systemen 29

3.1 Paraxiale Wellengleichung . . . . . . . . . . . . . . . . . . . . 29

3.1 .1 Gaußsche Strahlen . . . . . . . . . . . . . . . 30

3.1 .2 Höhere transversale Lasermoden . . . . . . . . . . . . . . 32

3.1 .3 Bestimmung des Strahlradius . . . . . . . . . . . . . . . 32

3.1.4 Fokussierbarkeit nicht-Gaußscher Strahlen . . . . . . . . 34

3.1.5 Das Kirchhoffsche Beugungsintegral . . . . . . . . . . . . 35 
3.1 .6 Aberrationen . . . . . . . . . . . . . . . . 36

3.2 Selbstfokussierung . . . . . . . . . . . . . . . . . . . . . . . . 37

3.2.1 Townes-Soliton und kritische Leistung . . . . . . . . . 37

3.2.2 Kollaps der nichtlinearen Schrödingergleichung . . . . . . 40

3.2 .3 Kollapsprofil . . . . . . . . . . . . . . . . . . . . . . . . 41

3.2.4 Brennweite der Selbstfokussierung . . . . . . . . . . . 42

3.2 .5 Selbstfokussierung von Pulsen . . . . . . . . . . . . . 42

3.2 .6 Filamente . . . . . . . . . . . . . . . . . . . . . 43

3.3 Angewendetes Verfahren zur Berechnung fokussierter Pulse . . . 43

3.3 .1 Pulsform vor dem Fokusbereich . . . . . . . . . . . . . 43

3.3.2 Pulsausbreitung im Fokusbereich . . . . . . . . . . . . 44

3.3 .3 Crank-Nicolson-Verfahren . . . . . . . . . . . . . . 44

3.4 Beispiel: Pulsaufspaltung . . . . . . . . . . . . . . . . . . 46

4 Prozesse der Plasmabildung und -dynamik 48

4.1 Überblick . . . . . . . . . . . . . . . . . . . . . . . . . . . . . . . . . . . 48

4.2 Keldysh-Theorie der Photoionisation . . . . . . . . . . . . 51

4.2.1 Wahrscheinlichkeit der Photoionisation . . . . . . . . . 51

4.2 .2 Muliphotonenabsorption . . . . . . . . . . . . . 52

4.2 .3 Tunnelionisation . . . . . . . . . . . . . . . . . . 53

4.2 .4 Berechnung der Ionisationsrate $\ldots \ldots \ldots$. . . . . . . 53

4.2.5 Intensitätsabnahme und Entstehung freier Elektronen . . 55

4.2.6 $\quad$ Bestimmung der Absorption bei Photoionisation . . . . . 56

4.3 Kaskadenionisation . . . . . . . . . . . . . . . . 56

4.3 .1 Das Drude-Modell . . . . . . . . . . . . . . . . . 56

$4.3 .2 \quad$ Plasmafrequenz und kritische Dichte . . . . . . . . . . 58

4.4 Diffusion und Rekombination . . . . . . . . . . . . . . . . 59

4.5 Ratengleichung . . . . . . . . . . . . . . . . . . . . . 60

4.6 Durchbruchsschwelle . . . . . . . . . . . . . . . . . . . . . 61

$4.7 \quad$ Beispiele . . . . . . . . . . . . . . . . . . . . . . . . . . . . . . . 61 
5 Pulsausbreitung und Plasmadynamik 64

5.1 Erweiterte Ausbreitungsgleichung . . . . . . . . . . . . . . . . 64

5.2 Numerisches Verfahren . . . . . . . . . . . . . . . 65

5.3 Aufbau des Vergleichsexperiments . . . . . . . . . . . . 66

5.4 Ergebnisse der Simulation . . . . . . . . . . . . 67

5.5 Vergleich mit experimentellen Daten . . . . . . . . . . . . . 70

5.6 Einfluss des Strahlprofils auf die Plasmabildung . . . . . . . . . 74

6 Zusammenfassung und Ausblick $\quad 76$

6.1 Zusammenfassung . . . . . . . . . . . . . . 76

6.2 Ausblick . . . . . . . . . . . . . . . . . . 77

\begin{tabular}{|ll}
\hline A Symbolverzeichnis & 78
\end{tabular}

A.1 Konstanten . . . . . . . . . . . . . . . . . . 78

A.2 Variable und sonstige Bezeichnungen . . . . . . . . . . . . 78

B Fouriertransformation und Faltungssatz 82

\begin{tabular}{ll}
\hline Literaturverzeichnis & 83
\end{tabular}

\begin{tabular}{lr}
\hline Danksagung & 89
\end{tabular}

\begin{tabular}{ll}
\hline Lebenslauf & 91
\end{tabular} 


\section{Einleitung}

In der heutigen Zeit werden gepulste Lasersysteme in vielen Bereichen der Industrie wie der Materialbearbeitung und der Medizintechnik sowie der Forschung verwendet.

Neben Energie und Wellenlänge der Laserpulse ist dabei insbesondere die Dauer der verwendeten Laserpulse von großer Bedeutung. Zusätzlich zu Lasern, deren Pulse eine Länge von einigen Nanosekunden haben, sowie Lasern mit Pulslängen im Picosekundenbereich erfreuen sich Systeme, deren Pulse eine Länge von einigen hundert Femtosekunden oder weniger haben, großer Beliebtheit.

Dies liegt vor allem daran, dass bei kürzeren Pulsen weniger Energie gebraucht wird, um Leistungen zu erreichen, die z. B. für das Ablaufen nichtlinearer Prozesse benötigt werden. Diese spielen insbesondere bei der Wechselwirkung der Laserstrahlung mit für die entsprechende Wellenlänge eigentlich transparenten Medien eine große Rolle. Die durch die nichtlinearen Effekte verursachte Änderung des Brechungsindex führt dazu, dass bei hohen Intensitäten, die in der Regel bei der Fokussierung erreicht werden können, doch Laserstrahlung absorbiert werden kann und ein Plasma entsteht. Man spricht in diesem Fall vom optischen Durchbruch. Durch das Einbringen von Energie kann man zum Beispiel Veränderungen im behandelten Material (Erzeugung von Wellenleiterstrukturen in Glas, Schneiden der Augenlinse bei der Laserlasik [36]) erzeugen. Im Falle einer transparenten Flüssigkeit führt die schlagartige Erwärmung des Mediums zu einer starken Expansion und damit zu einem Aufreißen der Flüssigkeit und der Erzeugung einer Kavitationsblase [53, 54, 52. Dieser Vorgang wird optische Kavitation genannt und stellt ein Verfahren dar, mit dem man zuverlässig reproduzierbar Kavitationsblasen erzeugen kann [33].

Der Vorteil bei der Benutzung von Femtosekundenpulsen im Gegensatz zu längeren Pulsen liegt dabei darin, dass durch die geringere nötige Energie ein kleineres Volumen behandelt werden kann, und es dabei zu geringeren Nebeneffekten wie Erhitzung kommt. Allerdings ist es nur sehr eingeschränkt möglich, ein beliebig kleines idealerweise kugelförmiges Volumen zu präparieren, in dem die Absorption der Laserenergie vonstatten geht. Vielmehr beobachtet man, dass das entstandene Plasma insbesondere bei kleinen Fokussierungswinkeln entlang der optischen Achse stark ausgedehnt ist. Im Rahmen dieser Arbeit wird u. a. diese Ausdehnung untersucht. 
Aufgrund der beschriebenen Bedeutsamkeit des optischen Durchbruches besteht ein großes wissenschaftliches Interesse an dessen Untersuchung. Dabei werden sowohl experimentelle als auch numerische Untersuchungen an verschiedenen transparenten Medien wie Luft, Glas und Wasser vorgenommen.

Die theoretischen Arbeiten reichen von recht grundlegenden Ansätzen beispielsweise zur Bestimmung der maximalen Elektronendichte im optischen Durchbruch aufgrund von Ratengleichungsmodellen [45, 80], bis hin zu gekoppelten Simulationen, die sowohl die Ausbreitung der Laserpulse in den betreffenden Medien als auch das entstehende Plasma und dessen Rückwirkung auf den Puls berücksichtigen [27, 4, 5.

Die Entwicklung einer solchen Simulation steht im Mittelpunkt der vorliegenden Arbeit. Neben Berechnungen zur Ausbreitung ultrakurzer Pulse sowie der Untersuchung der Wechselwirkungen der einzelnen beteiligten Mechanismen wird ein Vergleich mit experimentellen Ergebnissen vorgestellt.

Dazu werden in Kapitel 1 die theoretischen Grundlagen der Pulsausbreitung dargestellt und eine Gleichung zur Berechnung der Pulsausbreitung hergeleitet.

Kapitel 2 beschreibt die verwendete Methode zum Lösen dieser Gleichung. Anhand von Ergebnissen zur Ausbreitung von Pulsen in einer (räumlichen) Dimension werden Phänomene diskutiert, die bei der Pulsausbreitung in nichtlinearen Medien auftreten.

In Kapitel 3 wird die Berechnung auf räumlich ausgedehnte Pulse ausgeweitet und werden Beispiele vorgestellt.

Die Mechanismen, die bei der Plasmaentstehung und -dynamik eine Rolle spielen, werden in Kapitel 4 diskutiert und das verwendete Modell zur Untersuchung der Plasmadynamik wird beschrieben.

Im darauffolgenden Kapitel 5 wird dargestellt, wie die Berechnungen der Pulsausbreitung und der Plasmadynamik kombiniert werden können. Weiterhin werden die Ergebnisse dieser Rechnungen vorgestellt und mit experimentellen Daten verglichen.

$\mathrm{Zu}$ guter Letzt werden in Kapitel [6 die Ergebnisse zusammengefasst und wird ein Ausblick auf mögliche weitere Untersuchungen gegeben. 


\section{Kapitel 1}

\section{Theoretische Grundlagen der Pulsausbreitung}

Im ersten Kapitel werden unterschiedliche Methoden vorgestellt, um die Ausbreitung von ultrakurzen Laserpulsen in (zunächst als nichtionisierbar angenommenen) Medien berechnen zu können. Als Ausgangspunkt dienen die Maxwell-Gleichungen, die - zusammen mit geeigneten Annahmen über die Magnetisierbarkeit sowie die Polarisierbarkeit des betrachteten Mediums - die umfassendste Beschreibungsmöglichkeit bilden. Numerisch einfacher zu handhaben und daher weit verbreitet sind Verfahren auf Basis von Einhüllendengleichungen, zu denen u. a. Gleichungen vom Typ der nichtlinearen Schrödingergleichungen gehören. Die Herleitung einer solchen Gleichung, die die Basis für die in dieser Arbeit verwendeten Ausbreitungsgleichungen darstellen wird, aus den Maxwell-Gleichungen wird in diesem Kapitel beschrieben.

\subsection{Maxwell-Gleichungen}

Die Beschreibung der Pulsausbreitung beginnt mit den Maxwell-Gleichungen, die wie folgt geschrieben werden können [1]:

$$
\begin{array}{ll}
\nabla \cdot \mathbf{D}=\rho^{\prime} & \nabla \cdot \mathbf{B}=0 \\
\nabla \times \mathbf{E}=-\frac{\partial \mathbf{B}}{\partial t} & \nabla \times \mathbf{H}=\mathbf{j}+\frac{\partial \mathbf{D}}{\partial t} .
\end{array}
$$

Der Laserpuls wird dabei durch die elektrische Feldstärke E, die elektrische Flussdichte $\mathbf{D}$, die magnetische Feldstärke $\mathbf{H}$ sowie die magnetische Flussdichte B beschrieben. Bei vernachlässigbarer Magnetisierung $\left(\mathbf{B}=\mu_{0} \mathbf{H}\right)$, verschwindendem Verschiebungsstrom $(\mathbf{j}=0)$ und keinen vorhandenen freien Ladungen $\left(\rho^{\prime}=0\right)$ erhält man: 


$$
\begin{aligned}
\nabla \cdot \mathbf{D} & =0 & \nabla \cdot \mathbf{B} & =0 \\
\nabla \times \mathbf{E} & =-\mu_{0} \frac{\partial \mathbf{H}}{\partial t} & \nabla \times \mathbf{H} & =\frac{\partial \mathbf{D}}{\partial t} .
\end{aligned}
$$

In einem polarisierbaren Medium ergibt sich als Zusammenhang zwischen dem Feld E und der Flussdichte D:

$$
\mathbf{D}=\epsilon_{0} \mathbf{E}+\mathbf{P}_{1}+\mathbf{P}_{\mathrm{nl}}
$$

Dabei wurde die Polarisation bereits in die linearen und nichtlinearen Anteile $\mathbf{P}_{1}$ und $\mathbf{P}_{\mathrm{nl}}$ aufgeteilt. Die Bedeutung dieser beiden Anteile wird in den beiden folgenden Abschnitten 1.2 und 1.3 diskutiert.

\subsection{Lineare Polarisation}

Für den linearen Anteil der Polarisation $\mathbf{P}_{1}$ gilt mit der elektrischen Suszeptibilität $\chi$

$$
\mathbf{P}_{1}=\epsilon_{0} \chi \mathbf{E}
$$

In Gleichung (1.5) können dann die linearen Anteile zusammengefasst werden, es ergibt sich

$$
\begin{aligned}
\mathbf{D} & =\epsilon_{0} \mathbf{E}+\epsilon_{0} \chi \mathbf{E}+\mathbf{P}_{\mathrm{nl}} \\
& =\epsilon_{0}(1+\chi) \mathbf{E}+\mathbf{P}_{\mathrm{nl}} \\
& =\epsilon_{0} \epsilon \mathbf{E}+\mathbf{P}_{\mathrm{nl}}
\end{aligned}
$$

mit der Permittivität $\epsilon$. Diese ist - in einem isotropen und nicht magnetisierbaren Medium - mit dem Brechnungsindex $n$ über den Zusammenhang (s. Abschnitt 1.4)

$$
n^{2}=\epsilon
$$

verknüpft und im Allgemeinen von der Frequenz des elektrischen Feldes abhängig.

Der Brechungsindex von Wasser kann sehr genau über Näherungsformeln bestimmt werden [78, 79]. Abbildung 1.1 zeigt den Verlauf des Brechungsindex von Wasser für Licht mit einer Wellenlänge von $400 \mathrm{~nm}$ bis $1200 \mathrm{~nm}$ (blaue Kurve) für $25^{\circ} \mathrm{C}$ und Normaldruck. Für die in dieser Arbeit häufiger verwendete Wellenlänge $800 \mathrm{~nm}$ erhält man zum Beispiel:

$$
n_{\mathrm{H}_{2} \mathrm{O}} \approx 1.3281
$$




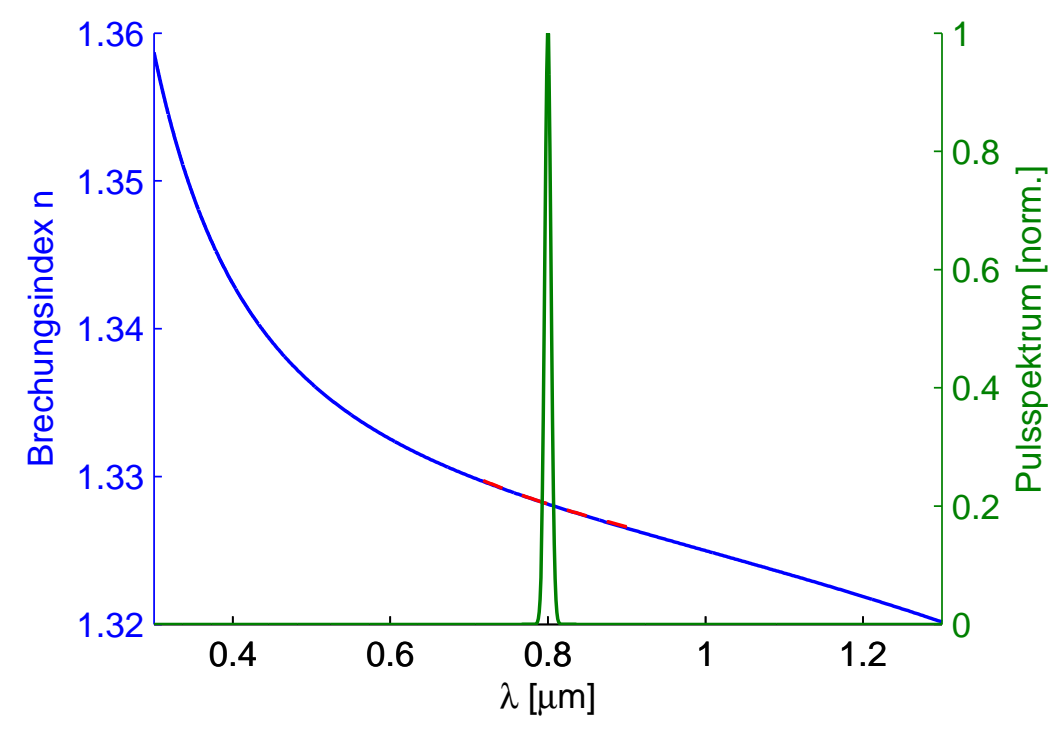

Abbildung 1.1: Verlauf des Brechnungsindex von Wasser für verschiedene Wellenlängen (berechnet nach [78, 79], blaue Kurve) und dessen lineare Approximation $\mathrm{dn} / \mathrm{d} \omega$ um $\lambda=800 \mathrm{~nm}$ (rot). Zum Vergleich das Leistungsspektrum eines 100 fs langen Pulses (vgl. Abschnitt 1.5.2, grün)

Analog zum Brechungsindex ist die Permittivität $\epsilon$ eine Funktion der Frequenz $\omega$. Das Produkt mit dem elektrischen Feld $\mathbf{E}$ erhält man über die Multiplikation im Frequenzraum, wobei $\tilde{\mathbf{E}}=\mathcal{F}(\mathbf{E})$ die Fouriertransformierte von E (vgl. Anhang B) bezeichnet:

$$
\tilde{\mathbf{P}}_{1}(\omega)=(\tilde{\epsilon} \cdot \tilde{\mathbf{E}})(\omega)
$$

Dies entspricht nach Gleichung (B.4 $)$ einer Faltung im Zeitbereich:

$$
\mathbf{P}_{l}(t)=(\epsilon * \mathbf{E})(t)=\int_{-\infty}^{t} d t^{\prime} \epsilon\left(t-t^{\prime}\right) \mathbf{E}(\mathbf{t})
$$

Über den Brechungsindex ist auch die Wellenzahl

$$
k(\omega)=\omega \cdot n(\omega) / c
$$

eine Funktion von $\omega$. Um die Auswirkung der Frequenzabhängigkeit der Wellenzahl zu untersuchen, betrachtet man deren Ableitungen nach $\omega$ am Ort $\omega_{0}$ :

$$
\begin{aligned}
\left.\frac{\partial k}{\partial \omega}\right|_{\omega_{0}} & =\left.\frac{n(\omega)}{c}\right|_{\omega_{0}}+\left.\frac{\omega}{c} \cdot \frac{d n}{d \omega}\right|_{\omega_{0}} \\
\left.\frac{\partial^{2} k}{\partial \omega^{2}}\right|_{\omega_{0}} & =\left.\frac{2}{c} \cdot \frac{d n}{d \omega}\right|_{\omega_{0}}+\left.\frac{\omega}{c} \frac{d^{2} n}{d \omega^{2}}\right|_{\omega_{0}} .
\end{aligned}
$$


Man erkennt anhand von Gleichung (1.11), dass sich die durch

$$
v_{\mathrm{g}}\left(\omega_{0}\right):=\left.\frac{\partial \omega}{\partial k}\right|_{\omega_{0}}
$$

definierte Gruppengeschwindigkeit $v_{\mathrm{g}}$ von der Phasengeschwindigkeit $v=c / n$ unterscheidet, wenn die Ableitung von $n$ nach $\omega$ an der Stelle $\omega_{0}$ von 0 verschieden ist. Gilt ferner $d n / d \omega>0$, so spricht man von einer normalen Dispersion, im Fall einer negativen Ableitung von einer anomalen Dispersion.

Für $d n / d \omega \neq 0$ nimmt auch die Gruppengeschwindigkeitsdispersion GVD (group velocity dispersion), die durch

$$
\operatorname{GVD}\left(\omega_{0}\right)=\left.\frac{\partial}{\partial \omega} \frac{1}{v_{\mathrm{g}}\left(\omega_{0}\right)}\right|_{\omega_{0}}=\left.\frac{\partial^{2} k}{\partial \omega^{2}}\right|_{\omega_{0}}
$$

gegeben ist, einen endlichen Wert an. Für eine Wellenlänge von $800 \mathrm{~nm}$ ergibt die Berechnung der GVD nach Gleichung (1.12) mit Hilfe des Brechungsindexverlaufs für Wasser nach [78, 79] (vgl. Abbildung 1.2) einen Wert von

$$
\mathrm{GVD}_{\mathrm{H}_{2} \mathrm{O}} \approx 24.24 \frac{\mathrm{fs}^{2}}{\mathrm{~mm}},
$$

der in guter Übereinstimmung zum experimentell bestimmten Wert GVD exp $=(25.71 \pm 0.5) \mathrm{fs}^{2} \mathrm{~mm}^{-1}$ [67] steht. Bei einem positiven Wert von GVD wird auch hier von einer normalen Gruppengeschwindigkeitsdispersion gesprochen.

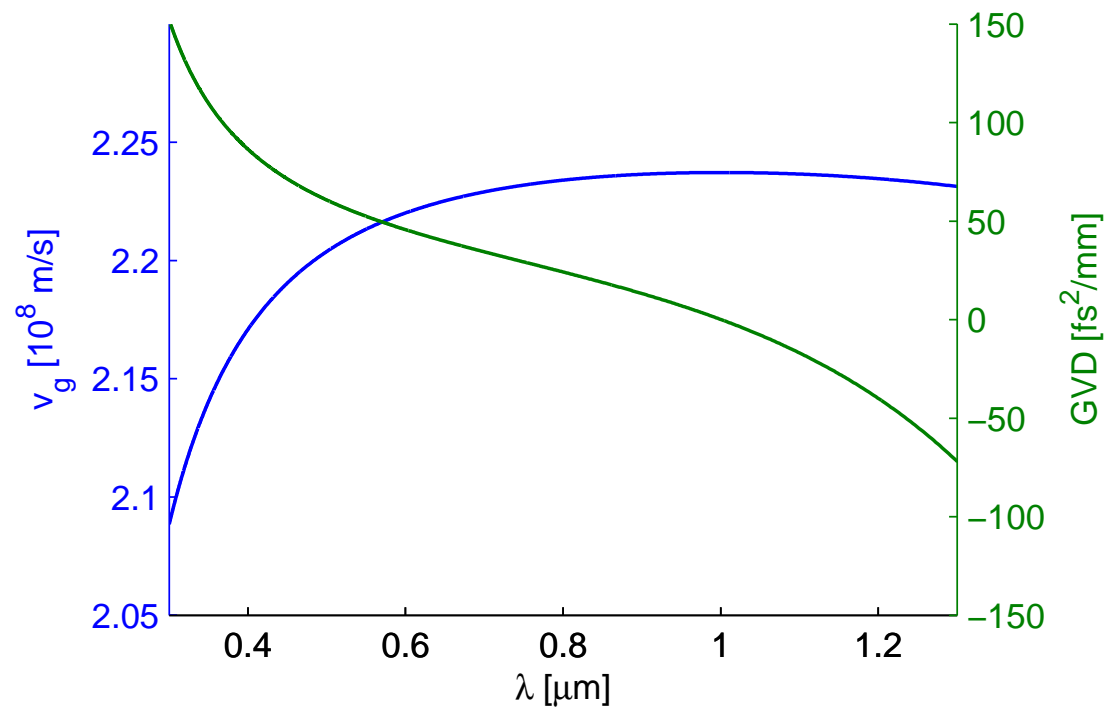

Abbildung 1.2: Gruppengeschwindigkeit $v_{\mathrm{g}}$ und Gruppengeschwindigkeitsdispersion GVD für Wasser in Abhängigkeit der Wellenlänge (berechnet aus dem Brechungsindexverlauf nach [78, 79] mit Hilfe der Gleichungen (1.14), (1.11) und (1.12) 
Bei der Berechnung der Pulsausbreitung verzichtet man oft auf die Berücksichtigung der Ableitungen weiterer höherer Ordnungen der Ableitung von $k$ nach $\omega$ (vgl. Abschnitt 1.5.2). In Abbildung 1.1 erkennt man, dass bereits eine lineare Approximation von $n(\omega)$ im Spektralbereich eines 100 fs langen Pulses die Brechungsindexkurve sehr gut widerspiegelt.

\subsection{Nichtlineare Polarisation: Kerr-Effekt}

Im vorherigen Kapitel wurde angenommen, dass die durch das angelegte Feld $\mathbf{E}$ induzierte Polarisation linear von diesem abhängt. Hebt man diese Einschränkung auf, so müssen bei der Berechnung der Polarisation $\mathbf{P}$ auch die Terme höherer Ordnung in $\mathbf{E}$ betrachtet werden:

$$
\mathbf{P}=\underbrace{\epsilon_{0} \chi^{(1)} \mathbf{E}}_{\mathbf{P}^{(1)}=\mathbf{P}_{1}}+\underbrace{\epsilon_{0}\left(\chi^{(2)} \mathbf{E}\right) \mathbf{E}}_{\mathbf{P}^{(2)}}+\underbrace{\epsilon_{0}\left(\left(\chi^{(3)} \mathbf{E}\right) \mathbf{E}\right) \mathbf{E}}_{\mathbf{P}^{(3)}}+\ldots
$$

Die Suszeptibilitäten höherer Ordnung $\chi^{(n)}$ sind im Allgemeinen Tensoren $(n+1)$-ter Stufe. Betrachtet man ein isotropes Medium wie Wasser, so können die Suszeptibilitäten aber - wie bisher - als skalar angesetzt werden.

Ferner besitzen isotrope Medien - wie auch viele Kristalle - eine sog. Inversionssymmetrie [1, 41], die zur Folge hat, dass bei Umkehrung der Richtung des elektrischen Feldes sich auch die Richtung der Polarisation umkehren muss. Damit verschwindet der Term 2. Ordnung $\mathbf{P}^{(2)}$ :

$$
\epsilon_{0}\left(\chi^{(2)} \mathbf{E}\right) \mathbf{E}=-\epsilon_{0}\left(\chi^{(2)}(-\mathbf{E})\right)(-\mathbf{E}) \Rightarrow \chi^{(2)}=-\chi^{(2)} \Rightarrow \chi^{(2)}=0 .
$$

Der niedrigste nichtlineare Term ist damit der Term 3. Ordnung. Die Terme höherer Ordnung werden im Allgemeinen vernachlässigt, so dass für den nichtlinearen Anteil der Polarisation $\mathbf{P}_{\mathrm{nl}}$ gilt:

$$
\mathbf{P}_{\mathrm{nl}}=\mathbf{P}^{(3)}=\epsilon_{0}\left(\left(\chi^{(3)} \mathbf{E}\right) \mathbf{E}\right) \mathbf{E}=\epsilon_{0} \chi^{(3)}|\mathbf{E}|^{2} \mathbf{E} .
$$

Wir betrachten im Folgenden ein Feld, dass mit der Frequenz $\omega_{0}$ und der Amplitude $\mathbf{E}_{0}$ schwingt:

$$
\mathbf{E}=\mathbf{E}_{0} \cos \left(\omega_{0} t\right)
$$

Für den nichtlinearen Anteil der Polarisation $\mathbf{P}_{\mathrm{nl}}$ erhält man:

$$
\mathbf{P}_{\mathrm{nl}}=\frac{3}{4} \epsilon_{0} \chi^{(3)}\left|\mathbf{E}_{0}\right|^{2} \mathbf{E}_{0} \cos \left(\omega_{0} t\right)+\frac{1}{4} \epsilon_{0} \chi^{(3)}\left|\mathbf{E}_{0}\right|^{2} \mathbf{E}_{0} \cos \left(3 \omega_{0} t\right) .
$$

Man erkennt, dass sich $\mathbf{P}_{\mathrm{nl}}$ aus zwei Anteilen zusammensetzt. Letzterer, der mit der dreifachen Frequenz der Anregung schwingt, kann in der Regel vernachlässigt werden, da sich die entstehende Welle im Allgemeinen nicht effizient ausbreiten kann. 
Für die Polarisation ergibt sich somit:

$$
\mathbf{P}=\epsilon_{0} \underbrace{\left(\chi^{(1)}+\frac{3}{4} \chi^{(3)}\left|\mathbf{E}_{0}\right|^{2}\right)}_{\chi_{\mathrm{ges}}=\chi_{1}+\chi_{\mathrm{n} 1}} \mathbf{E}_{0} \cos \left(\omega_{0} t\right) .
$$

Wie man sieht, erhält man die gesamte (effektive) Suszeptibilität $\chi_{\text {ges }}$ durch Addition des linearen $\chi_{1}$ und des nichtlinearen Anteils $\chi_{\mathrm{nl}}$. Analoges gilt für den Brechungsindex, wenn man annehmen kann, dass $\chi_{\mathrm{nl}}$ klein im Vergleich $\mathrm{zu} 1+\chi_{1}$ ist:

$$
n_{\mathrm{ges}}=\sqrt{\epsilon_{\mathrm{ges}}}=\sqrt{1+\chi_{\mathrm{ges}}}=\sqrt{1+\chi^{(1)}+\frac{3}{4} \chi^{(3)}\left|\mathbf{E}_{0}\right|^{2}} \approx n_{0}+\frac{3}{8 n_{0}} \chi^{(3)}\left|\mathbf{E}_{0}\right|^{2} .
$$

Der Gesamtbrechungsindex ergibt sich also aus der Summe des linearen Brechungsindex und einem Anteil, der proportional zum Quadrat der Amplitude des angelegten Feldes ist. In Abhängigkeit von der Intensität] erhält man

$$
n_{\text {ges }}=n_{0}+\underbrace{\frac{3}{4 n_{0}^{2} c \epsilon_{0}} \chi^{(3)}}_{n_{2}} I .
$$

Für Wasser existieren für $n_{2}$ verschiedene experimentell bestimmte Werte [76], in der vorliegenden Arbeit wird

$$
n_{2}=2 \cdot 10^{-16} \frac{\mathrm{cm}^{2}}{\mathrm{~W}}
$$

angenommen [39]. $n_{2}$ ist im Allgemeinen abhängig von der Pulslänge des eingestrahlten Lichts, da unterschiedliche Mechanismen für die Entstehung der Nichtlinearität verantwortlich sein können [76]. Für fs-Pulse kann angenommen werden, dass die Nichtlinearität ausschließlich aus einer nichtlinearen Verzerrung der Elektronenhülle durch das eingestrahlte elektrische Feld resultiert. Dessen Einfluss kann als instantan wirkend betrachtet werden.

Ferner können nichtlineare Effekte wie der Raman-Effekt [1], bei denen die Wirkung des Pulses erst zeitverzögert eintritt, bei der Betrachtung von fsPulsen in Wasser vernachlässigt werden [27].

\subsection{Wellengleichung}

Wendet man auf beide Seiten von Gleichung (1.3) den Rotationsoperator an, so ergibt sich [1]:

$$
\nabla \times \nabla \times \mathbf{E}=-\mu_{0} \frac{\partial(\nabla \times \mathbf{H})}{\partial t} .
$$

\footnotetext{
${ }^{1}$ vgl. Gleichung (1.32); die dort auftretende Einhüllende stellt eine Erweiterung der Amplitude $E_{0}$ dar.
} 
Einsetzen von Gleichung (1.4) in (1.20) liefert:

$$
\nabla \times \nabla \times \mathbf{E}=-\mu_{0} \frac{\partial^{2} \mathbf{D}}{\partial t^{2}}
$$

Mit der Vektoridentität

$$
\nabla \times(\nabla \times \mathbf{E})=\nabla(\nabla \cdot \mathbf{E})-\triangle \mathbf{E}
$$

ergibt sich bei Verwendung der Gleichungen (1.1) und (1.7):

$$
\triangle \mathbf{E}=\mu_{0} \frac{\partial^{2} \mathbf{D}}{\partial t^{2}}
$$

Mit dem Ansatz für D aus Gleichung (1.5) und der Vakuumlichtgeschwindigkeit $c$, die gegeben ist durch

$$
c=\frac{1}{\sqrt{\epsilon_{0} \mu_{0}}},
$$

erhält man die folgende Wellengleichung:

$$
\triangle \mathbf{E}=\frac{1}{c^{2}} \frac{\partial^{2}}{\partial t^{2}}(\epsilon \mathbf{E})+\mu_{0} \frac{\partial^{2}}{\partial t^{2}} \mathbf{P}_{\mathrm{nl}} .
$$

Im allgemeinen Fall, dass $\epsilon$ wie in Abschnitt 1.2 beschrieben von der Frequenz des einstrahlenden Lichtes abhängt, ergibt sich mit Gleichung (1.9):

$$
\triangle \mathbf{E}(t)-\frac{1}{c^{2}} \frac{\partial^{2}}{\partial t^{2}} \int_{-\infty}^{t} d t^{\prime} \epsilon\left(t-t^{\prime}\right) \mathbf{E}(t)=\mu_{0} \frac{\partial^{2}}{\partial t^{2}} \mathbf{P}_{\mathrm{nl}}(t)
$$

Ferner erkennt man in Gleichung (1.23) sofort den bereits erwähnten Zusammenhang

$$
n^{2}=\epsilon
$$

\subsection{Lösungsmethoden auf Grundlage von kom- plexen Einhüllenden}

\subsubsection{Die komplexe Einhüllende eines ultrakurzen Laser- pulses}

Anstelle der direkten Lösung der Maxwell-Gleichungen für die Berechnung der Ausbreitung von Laserpulsen werden oft Näherungsgleichungen auf Basis der sog. komplexen Einhüllenden verwendet [1, 70]. Die Herleitung einer solchen Gleichung, die sich an dem Vorgehen von Brabec und Krausz [10] orientiert, wird im Folgenden beschrieben. 
Dazu wird das elektrische Feld $E 2$ an einem Ort $\mathbf{r}$ und zur Zeit $t$ durch den Realteil des komplexen Feldes $\hat{E}(\mathbf{r}, t)$ dargestellt, es gilt:

$$
E(\mathbf{r}, t)=\operatorname{Re}(\hat{E}(\mathbf{r}, t))=\frac{1}{2}(\hat{E}(\mathbf{r}, t)+\text { c. c. }) .
$$

Nun wählt man für $\hat{E}(\mathbf{r}, t)$ den folgenden Ansatz

$$
\hat{E}(\mathbf{r}, t)=A(\mathbf{r}, t) e^{i\left(\beta_{0} z-\omega_{0} t+\psi_{0}\right)}
$$

mit der komplexen Einhüllenden $A(\mathbf{r}, t)$ des elektrischen Feldes. $\omega_{0}$ stellt dabei die mittlere Frequenz bezogen auf die spektrale Leistungsdichte des Laserpulses in der Startebene $\mathbf{r}_{\mathbf{0}}$ dar:

$$
\omega_{0}=\frac{\int_{-\infty}^{\infty} d \omega \omega|\tilde{E}(\omega)|^{2}}{\int_{-\infty}^{\infty} d \omega|\tilde{E}(\omega)|^{2}} .
$$

Ferner bezeichnet $\beta_{0}$, das gegeben ist durch

$$
\beta_{0}=\operatorname{Re}\left[k\left(\omega_{0}\right)\right]=\left(\omega_{0} / c\right) n_{0} \quad \operatorname{mit} \quad n_{0}=n\left(\omega_{0}\right),
$$

den Realteil der Wellenzahl $k$ bezogen auf die mittlere Frequenz $\omega_{0}$. Die Phase $\psi_{0}$ wird so gewählt, dass $A(\mathbf{r}, t)$ für $\mathbf{r}=\mathbf{0}$ und $t=0$ reellwertig wird.

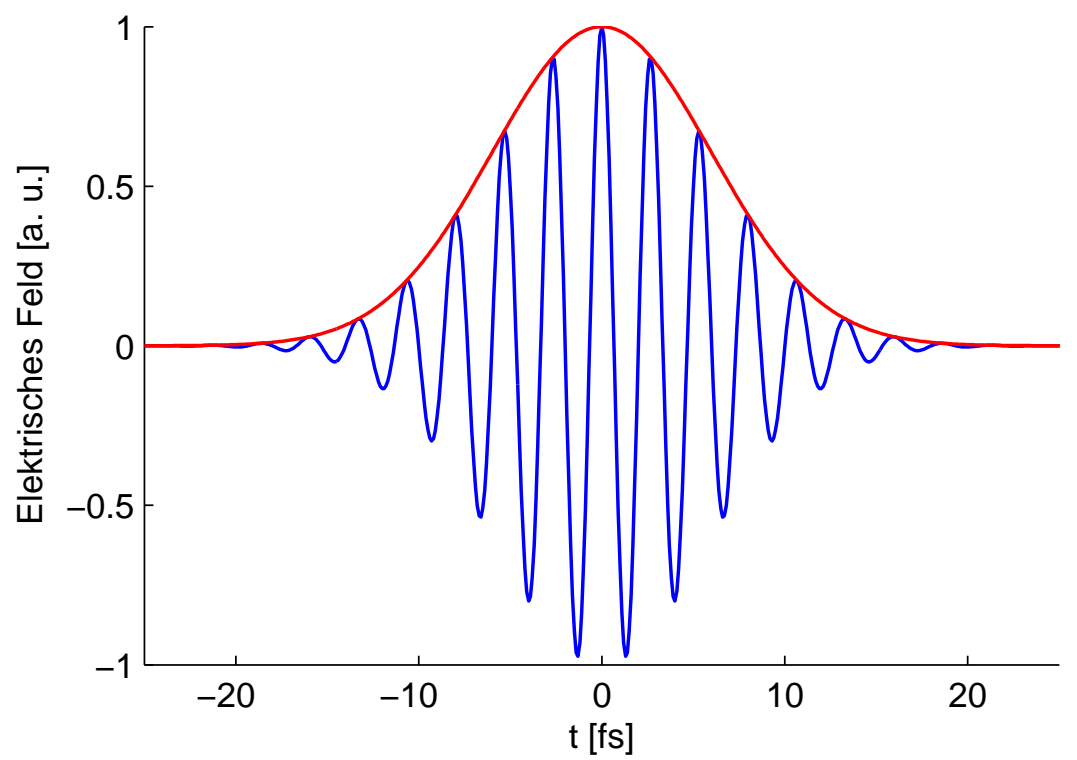

Abbildung 1.3: Elektrisches Feld (blau) und Einhüllende (rot) eines Pulses der Dauer $t_{\mathrm{FWHM}}=10 \mathrm{fs}$ und einer Wellenlänge von $\lambda=800 \mathrm{~nm}$.

\footnotetext{
${ }^{2}$ Das elektrische Feld $\mathbf{E}$ wird im Folgenden als linear polarisiert angenommen und kann daher als skalar angesetzt werden.
} 
Abbildung 1.3 zeigt beispielhaft die komplexe Einhüllende für einen $10 \mathrm{fs}$ langen Laserpuls. Man erkennt sofort, dass für Pulse, die viele Perioden der mittleren Frequenz enthalten, in der numerischen Behandlung die Darstellung über die komplexe Einhüllende von großem Vorteil ist, da für deren Diskretisierung wesentlich weniger Punkte benötigt werden als für die des elektrischen Feldes.

Der allgemeine komplexwertige Ansatz für die Einhüllende ist nötig, um auch Pulse darstellen zu können, in deren zeitlichem Verlauf sich die Frequenz verändert (Chirp, [1]). Im einfachsten Fall wird die Einhüllende reellwertig in Form eines sech- oder Gauß-Pulses angesetzt:

$$
\begin{array}{ll}
\text { sech-Puls: } & A(t)=A_{0} \operatorname{sech}\left(\frac{t}{t_{0}}\right), \\
\text { Gauß-Puls: } & A(t)=A_{0} \exp \left(-\frac{t^{2}}{t_{0}^{2}}\right) .
\end{array}
$$

Dabei wird die Pulsdauer jeweils durch $t_{0}$ vorgegeben. Üblicher ist es, die zeitliche Länge auf die Zeitspanne zwischen dem Anstieg bzw. Abfall des Laserpulses auf die Hälfte seiner Intensität (vgl. Gleichung (1.32) ) zu beziehen (Full Width at Half Maximum, FWHM), wie es im Folgenden in dieser Arbeit - wenn nicht explizit etwas anderes angegeben ist - gemacht wird. Für die betrachteten Pulsformen findet man für den Zusammenhang zwischen dieser Zeitdauer $t_{\mathrm{FWHM}}$ und der jeweiligen Zeit $t_{0}$ [71]:

$$
\begin{aligned}
\text { sech-Puls: } & t_{0}=\frac{t_{\mathrm{FWHM}}}{2 \operatorname{acosh}(\sqrt{2})}, \\
\text { Gauß-Puls: } & t_{0}=\frac{t_{\mathrm{FWHM}}}{\sqrt{2 \ln 2}} .
\end{aligned}
$$

Dabei ist die Intensität des Laserpulses wie folgt definiert:

$$
I(t)=\frac{1}{2} n_{0} c \epsilon_{0}|A(t)|^{2}
$$

Die Leistung des Laserpulses (zu einem gegebenen Zeitpunkt $t$ ) in der Ebene, die die Ausbreitungsrichtung an der Stelle $z$ senkrecht schneidet, ist gegeben durch die Integration der Intensität über diese Ebene:

$$
\mathrm{P}(z, t)=\int_{-\infty}^{\infty} \int_{-\infty}^{\infty} d x d y I(x, y, z, t)=\int_{0}^{2 \pi} d \phi \int_{0}^{\infty} d r r I(\phi, r, z, t) .
$$

Für einen Puls, dessen räumliche Ausdehnung durch ein gaufförmiges Strahlprofil beschrieben wird (vgl. Abschnitt 3.1), erhält man:

$$
\mathrm{P}_{\text {gauss }}(z, t)=\pi \int_{-\infty}^{\infty} d r r I_{0}(z, t) \exp \left(-\frac{2 r^{2}}{w^{2}(z, t)}\right)=\frac{\pi}{2} I_{0}(z, t) w^{2}(z, t)
$$


Durch zeitliche Integration der Leistung kann die Energie E des Laserpulses bestimmt werden:

$$
\mathrm{E}(z)=\int_{-\infty}^{\infty} d t \mathrm{P}(z, t)
$$

Es ergibt sich mit $\mathrm{P}_{0}(z)=\mathrm{P}(z, t=0)$ für die betrachteten Pulsformen (vgl. Gleichungen (1.29) und (1.28) ) :

$$
\begin{aligned}
& \text { sech-Puls: } \quad \mathrm{E}(z)=\int_{-\infty}^{\infty} d t \mathrm{P}_{0}(z) \exp \left(-\frac{2 t^{2}}{t_{0}^{2}(z)}\right)=\sqrt{\frac{\pi}{2}} \mathrm{P}_{0}(z) t_{0}(z), \\
& \text { Gauß-Puls: } \quad \mathrm{E}(z)=\int_{-\infty}^{\infty} d t \mathrm{P}_{0}(z) \operatorname{sech}^{2}\left(\frac{t}{t_{0}(z)}\right)=2 \mathrm{P}_{0}(z) t_{0}(z) .
\end{aligned}
$$

\subsubsection{Herleitung der Einhüllendengleichung}

Nach der Einführung des Begriffs der komplexen Einhüllenden wird in diesem Abschnitt eine Gleichung vorgestellt, die die Ausbreitung von Pulsen mit Hilfe der komplexen Einhüllenden beschreibt. Die Herleitung dieser Einhüllendengleichung aus der Wellengleichung orientiert sich dabei an dem Vorgehen von Brabec und Krausz [10].

Zunächst wird analog zur Einhüllenden des elektrischen Feldes nach Gleichung (1.26) die Einhüllende der nichtlinearen Polarisation $B(\mathbf{r}, t, A)$ definiert:

$$
P_{\mathrm{nl}}(\mathbf{r}, t)=\operatorname{Re}\left(B(\mathbf{r}, t, A) e^{i\left(\beta_{0} z-\omega_{0} t+\psi\right)}\right) .
$$

Diese ist insbesondere eine Funktion der Einhüllenden des elektrischen Feldes $A(\mathbf{r}, t)$, der Zusammenhang ist im Allgemeinen natürlich nichtlinear 3

Das weitere Vorgehen besteht nun zunächst darin, die Gleichungen (1.26) und (1.35), in denen die Einhüllenden des elektrischen Feldes sowie der nichtlinearen Polarisation definiert wurden, in die Wellengleichung (1.24) einzusetzen. Die einzelnen dabei auftretenden Terme werden im Folgenden hergeleitet.

Mit

$$
\begin{aligned}
\partial_{z}^{2} E(\mathbf{r}, t) & =\partial_{z}^{2}\left(A(\mathbf{r}, t) \cdot e^{i\left(\beta_{0} z-\omega_{0} t+\psi_{0}\right)}\right) \\
& =\left(\partial_{z}^{2}+2 i \beta_{0} \partial_{z}-\beta_{0}^{2}\right) \cdot A(\mathbf{r}, t) e^{i\left(\beta_{0} z-\omega_{0} t+\psi_{0}\right)}
\end{aligned}
$$

ergibt sich für den ersten Term von Gleichung (1.24):

$$
\left(\partial_{z}^{2}+\nabla_{\perp}^{2}\right) E(\mathbf{r}, t)=\left(\partial_{z}^{2}+2 i \beta_{0} \partial_{z}-\beta_{0}^{2}+\nabla_{\perp}^{2}\right) A(\mathbf{r}, t) \cdot e^{i\left(\beta_{0} z-\omega_{0} t+\psi_{0}\right)} .
$$

\footnotetext{
${ }^{3}$ Das elektrische Feld $E$ und die nichtlineare Polarisation $P_{\mathrm{nl}}$ sowie deren Einhüllende sind stets als Funktionen von $\mathbf{r}$ und $\mathbf{t}$ aufzufassen, zur Vereinfachung der Schreibweise werden diese Abhängigkeiten im Folgenden zum Teil weggelassen.
} 
Dabei bezeichnet $\mathbf{r}_{\perp}$ den Anteil von $\mathbf{r}$ senkrecht zur Ausbreitungsrichtung $z$ und entsprechend $\triangle_{\perp}=\nabla_{\perp}^{2}=\partial_{x}^{2}+\partial_{y}^{2}$ den entsprechenden Anteil des LaplaceOperators.

Der zweite Term von Gleichung (1.24) stellt eine Faltung dar, so dass man mit Hilfe der Definition der inversen Fouriertransformation (B.2), des Faltungssatzes (B.4) sowie der Gleichungen (1.25) und (1.10) schreiben kann:

$$
\begin{aligned}
-\frac{1}{c^{2}} \partial_{t}^{2} \int_{-\infty}^{t} d t^{\prime} \epsilon\left(t-t^{\prime}\right) E\left(t^{\prime}\right)= \\
\quad=-\frac{1}{c^{2}} \partial_{t}^{2} \frac{1}{2 \pi} \int_{-\infty}^{\infty} d \omega e^{i \omega t} \tilde{\epsilon}(\omega) \tilde{E}(\omega) \\
=\frac{1}{2 \pi} \int_{-\infty}^{\infty} d \omega e^{i \omega t} \tilde{k}^{2}(\omega) \tilde{E}(\omega) \\
=\frac{1}{2 \pi} \int_{-\infty}^{\infty} d \omega e^{i \omega t}\left(\left.\sum_{m=0}^{\infty} \frac{\partial^{m} \tilde{k}}{\partial w^{m}}\right|_{\omega_{0}} \cdot\left(\omega-\omega_{0}\right)^{m}\right)^{2} \tilde{E}(\omega) .
\end{aligned}
$$

Im letzten Schritt wurde dabei die Taylorentwicklung von $\tilde{k}(\omega)$ um den Punkt $\omega_{0}$ eingesetzt. Mit Hilfe von $\mathcal{F}\left(\partial_{t}^{n} f(t)\right)=(i \omega)^{n} \mathcal{F}(f(t))$ und Gleichung (1.26) ergibt sich für den Ausdruck (1.37):

$$
\begin{array}{r}
\frac{1}{2 \pi} \int_{-\infty}^{\infty} d \omega e^{i \omega t}\left(\sum_{m=0}^{\infty} \frac{\left.\frac{\partial^{m} \tilde{k}}{\partial w^{m}}\right|_{\omega_{0}}}{m !} \cdot\left(\omega-\omega_{0}\right)^{m}\right)^{2} \tilde{E}(\omega) \\
=\left(\sum_{m=0}^{\infty} \frac{\left.\frac{\partial^{m} \tilde{k}}{\partial w^{m}}\right|_{\omega_{0}}}{m !} \cdot\left(i \partial_{t}\right)^{m}\right)^{2} A(t) \cdot e^{i\left(\beta_{0} z-\omega_{0} t+\psi_{0}\right)} .
\end{array}
$$

Teilt man die Ableitungen von $\tilde{k}$ nach $\omega$ in Terme der Absorption $\alpha_{m}$ und der Dispersion $\beta_{m}$ mit

$$
\alpha_{m}=\operatorname{Im}\left(\left.\frac{\partial^{m} \tilde{k}}{\partial \omega^{m}}\right|_{\omega_{0}}\right) \quad \text { und } \quad \beta_{m}=\operatorname{Re}\left(\left.\frac{\partial^{m} \tilde{k}}{\partial \omega^{m}}\right|_{\omega_{0}}\right)
$$

auf, so kann man die Summe in

$$
\sum_{m=0}^{\infty} \frac{\left.\frac{\partial^{m} \tilde{k}}{\partial w^{m}}\right|_{\omega_{0}}}{m !} \cdot\left(i \partial_{t}\right)^{m}=\sum_{m=0}^{\infty} \frac{\beta_{m}+i \frac{\alpha_{m}}{2}}{m !} \cdot\left(i \partial_{t}\right)^{m}=\beta_{0}+i \frac{\alpha_{0}}{2}+i \beta_{1} \partial_{t}+\hat{D}
$$

umformen. Dabei tritt der Dispersionsoperator $\hat{D}$ auf, für den gilt:

$$
\hat{D}=-\frac{\alpha_{1}}{2} \partial_{t}+\sum_{m=2}^{\infty} \frac{\beta_{m}+i \frac{\alpha_{m}}{2}}{m !} \cdot\left(i \partial_{t}\right)^{m}
$$


Den letzten fehlenden Term aus der Wellengleichung (1.24) erhält man - unter Verwendung der komplexen Einhüllenden für die nichtlineare Polarisation (vgl. Gleichung (1.35) ) - wie folgt:

$$
\begin{aligned}
\mu_{0} \partial_{t}^{2}\left(B \cdot e^{i\left(\beta_{0} z-\omega_{0} t+\psi_{0}\right)}\right) & =\mu_{0}\left(\partial_{t}^{2}-2 i \omega_{0} \partial_{t}-\omega_{0}^{2}\right) B e^{i\left(\beta_{0} z-\omega_{0} t+\psi_{0}\right)} \\
& =-\mu_{0} \omega_{0}^{2}\left(1+\frac{i}{\omega_{0}} \partial_{t}\right)^{2} B e^{i\left(\beta_{0} z-\omega_{0} t+\psi_{0}\right)}
\end{aligned}
$$

Fasst man die Ergebnisse für die einzelnen Terme aus den Gleichungen (1.36), (1.38) und (1.42) zusammen, so ergibt sich:

$$
\begin{aligned}
\left(\partial_{z}^{2}+2 i \beta_{0} \partial_{z}-\beta_{0}^{2}+\nabla_{\perp}^{2}\right) A+\left(\beta_{0}+i \frac{\alpha_{0}}{2}+i \beta_{1} \partial_{t}+\hat{D}\right)^{2} A & \\
& =\mu_{0} \omega_{0}^{2}\left(1+\frac{i}{\omega_{0}} \partial_{t}\right)^{2} B
\end{aligned}
$$

Dabei wurde die gesamte Gleichung durch den Faktor $e^{i\left(\beta_{0} z-\omega_{0} t+\psi_{0}\right)}$ geteilt und Gleichung (1.40) verwendet.

Wenn man bedenkt, dass ein Laserpuls von 100 fs Länge eine räumliche Ausdehnung von ungefähr $30 \mu \mathrm{m}$ besitzt, die Ausbreitung aber über eine Strecke von einigen Zentimetern oder länger gerechnet werden soll, dann wird deutlich, dass die Berechnung nur effizient in einem mitbewegten Koordinatensystem durchgeführt werden kann. Ferner bietet es sich - aufgrund der auftretenden hohen zeitlichen Ableitungen in $\hat{D}$ (vgl. Gleichung (1.41) ) - an, bei jedem Schritt der Berechnung den Pulsverlauf an einem Ort für alle (relevanten) Zeiten zu bestimmen (und nicht etwa umgekehrt wie es sicherlich zunächst intuitiver wäre).

Allerdings werden bei diesem Vorgehen keine zurücklaufenden Wellen berücksichtigt. Dies ist insbesondere bei der Berechnung der Ausbreitung in ionisierbaren Medien (s. Kapitel 4) zu beachten, da das entstehende Plasma bei entsprechender Dichte reflektierend wirkt.

Legt man dieses Vorgehen fest, so ergibt sich der Übergang in ein mit der Gruppengeschwindigkeit $\beta_{1}^{-1}$ (vgl. Gleichung (1.13) ) mitgeführtes Koordinatensystem $(\tau, \xi)$ durch:

$$
\tau=t-\beta_{1} z \quad \text { und } \quad \xi=z .
$$

Um die Ausbreitungsgleichung (1.43) in den neuen Koordinaten schreiben zu können, werden zunächst die partiellen Ableitungen von $A$ nach den alten Koordinaten als Funktion der Ableitungen nach den neuen Koordinaten berechnet:

$$
\begin{aligned}
\partial_{t} A & =\partial_{\tau} A, & \partial_{t}^{2} A & =\partial_{\tau}^{2} A, \quad \ldots \\
\partial_{z} A & =\partial_{\xi} A-\beta_{1} \partial_{\tau} A, & \partial_{z}^{2} A & =\partial_{\xi}^{2} A-2 \beta_{1} \partial_{\xi} \partial_{\tau} A+\beta_{1}^{2} \partial_{\tau}^{2} A .
\end{aligned}
$$


Damit erhält man für den linken Teil von Gleichung (1.43) (anzuwenden auf A):

$$
\begin{gathered}
-\beta_{0}^{2}+2 i \beta_{0} \partial_{z}+\partial_{z}^{2}+\triangle_{\perp}+\beta_{0}^{2}+i \alpha_{0} \beta_{0}+2 i \beta_{0} \beta_{1} \partial_{\tau}+2 \beta_{0} \hat{D} \\
-\frac{\alpha_{0}^{2}}{4}-\alpha_{0} \beta_{1} \partial_{\tau}+i \alpha_{0} \hat{D}-\beta_{1}^{2} \partial_{\tau}^{2}+2 i \beta_{1} \hat{D} \partial_{\tau}+\hat{D}^{2} \\
=2 i \beta_{0} \partial_{\xi}-2 i \beta_{0} \beta_{1} \partial_{\tau}+\partial_{\xi}^{2}-2 \beta_{1} \partial_{\xi} \partial_{\tau}+\beta_{1}^{2} \partial_{\tau}^{2}+\triangle_{\perp}+i \alpha_{0} \beta_{0} \\
+2 i \beta_{0} \beta_{1} \partial_{\tau}+2 \beta_{0} \hat{D}-\frac{\alpha_{0}^{2}}{4}-\alpha_{0} \beta_{1} \partial_{\tau}+i \alpha_{0} \hat{D}-\beta_{1}^{2} \partial_{\tau}^{2}+2 i \beta_{1} \hat{D} \partial_{\tau}+\hat{D}^{2} \\
=2 i \beta_{0} \partial_{\xi}+i \alpha_{0} \beta_{0}+2 \beta_{0} \hat{D}-\frac{2 \beta_{0}}{\omega_{0}} \partial_{\tau} \partial_{\xi}+\frac{\alpha_{0} \beta_{0}}{\omega_{0}} \partial_{\tau}+\frac{2 i \beta_{0}}{\omega_{0}} \hat{D} \partial_{\tau} \\
\quad+\frac{2 \beta_{0}}{\omega_{0}} \partial_{\tau} \partial_{\xi}-\frac{\alpha_{0} \beta_{0}}{\omega_{0}} \partial_{\tau}-\frac{2 i \beta_{0}}{\omega_{0}} \hat{D} \partial_{\tau}-2 \beta_{1} \partial_{\xi} \partial_{\tau}-\alpha_{0} \beta_{1} \partial_{\tau}+2 i \beta_{1} \hat{D} \partial_{\tau} \\
+\partial_{\xi}^{2}+\hat{D}^{2}+i \alpha_{0} \hat{D}-\frac{\alpha_{0}^{2}}{4}-\alpha_{0} \beta_{1} \partial_{\tau}+\triangle_{\perp} \\
=2 i \beta_{0}\left(1+\frac{i}{\omega_{0}} \partial_{\tau}\right)\left(\partial_{\xi}+\frac{\alpha_{0}}{2}-i \hat{D}\right) \\
\quad-2 i \beta_{0}\left(\frac{\beta_{0}-\omega_{0} \beta_{1}}{\beta_{0}}\right) \frac{i}{\omega_{0}} \partial_{\tau}\left(\partial_{\xi}+\frac{\alpha_{0}}{2}-i \hat{D}\right) \\
+\partial_{\xi}^{2}+\hat{D}^{2}+i \alpha_{0} \hat{D}-\frac{\alpha_{0}^{2}}{4}-\alpha_{0} \beta_{1} \partial_{\tau}+\triangle_{\perp} .
\end{gathered}
$$

Es ergibt sich für die Ausbreitungsgleichung (1.43):

$$
\begin{gathered}
\left(1+\frac{i}{\omega_{0}} \partial_{\tau}\right)\left[\left(\partial_{\xi}+\frac{\alpha_{0}}{2}-i \hat{D}\right) A+\frac{\mu_{0} \omega_{0}^{2}}{2 i \beta_{0}}\left(1+\frac{i}{\omega_{0}} \partial_{\tau}\right) B\right]+\frac{1}{2 i \beta_{0}} \triangle_{\perp} A \\
=\left(\frac{\beta_{0}-\omega_{0} \beta_{1}}{\beta_{0}}\right) \frac{i}{\omega_{0}} \partial_{\tau}\left(\partial_{\xi}+\frac{\alpha_{0}}{2}-i \hat{D}\right) A \\
\quad-\frac{1}{2 i \beta_{0}}\left(\partial_{\xi}^{2}+\hat{D}^{2}+i \alpha_{0} \hat{D}-\frac{\alpha_{0}^{2}}{4}-\alpha_{0} \beta_{1} \partial_{\tau}\right) A
\end{gathered}
$$

Dabei wurde so umgeformt, dass die Terme der rechten Seite von Gleichung (1.46) vernachlässigt werden können, wenn sich die Einhüllende $A$ im Verlauf der Propagation im Vergleich zur Wellenlänge bzw. zur Periodendauer nur wenig verändert:

$$
\left|\partial_{\xi} A\right| \ll \beta_{0}|A| \quad \text { und } \quad\left|\partial_{\tau} A\right| \ll \omega_{0}|A| \text {. }
$$

Diese Bedingungen ergeben sich mit $\omega_{0} \approx \beta_{0} / \beta_{1}$ aus der sogenannten slowlyvarying-envelope approximation (SVEA) [10]:

$$
\left|\partial_{z} A\right|=\left|\partial_{\xi} A-\beta_{1} \partial_{\tau} A\right| \ll \beta_{0}|A| .
$$

Diese bedeutet anschaulich, dass sich die Einhüllende $A$ im Verlauf der Propagation im Vergleich zur Wellenlänge bzw. zur Periodendauer nur wenig verändert. Ferner sieht man, dass die SVEA die paraxiale Näherung [71] mit 
einschließt:

$$
\left|\frac{\partial^{2} A}{\partial z^{2}}\right| \ll\left|2 \beta_{0} \frac{\partial A}{\partial z}\right| \quad \text { oder } \quad\left|\frac{\partial^{2} A}{\partial z^{2}}\right| \ll \triangle_{\perp} A
$$

Mit den Näherungen (1.47) erhält man aus Gleichung (1.46):

$$
\begin{aligned}
\partial_{\xi} A= & -\frac{\alpha_{0}}{2} A+i \hat{D} A+\frac{i}{2 \beta_{0}}\left(1+\frac{i}{\omega_{0}} \partial_{\tau}\right)^{-1} \triangle_{\perp} A \\
& +i \frac{\mu_{0} \omega_{0}^{2}}{2 \beta_{0}}\left(1+\frac{i}{\omega_{0}} \partial_{\tau}\right) B .
\end{aligned}
$$

Fasst man die komplexe Einhüllende $A$ als Verallgemeinerung der in Gleichung (1.16) eingeführten Amplitude $E_{0}$ auf, so ergibt sich mit den Gleichungen (1.17) und (1.18) für die Einhüllende der nichtlinearen Polarisation:

$$
B=\epsilon_{0} \frac{3}{4} \chi^{(3)}|A|^{2} A=n_{0}^{2} c \epsilon_{0}^{2} n_{2}|A|^{2} A .
$$

Die Einhüllendengleichung (1.50) wird damit zu:

$$
\begin{aligned}
\partial_{\xi} A= & -\frac{\alpha_{0}}{2} A+i \hat{D} A+\frac{i}{2 \beta_{0}}\left(1+\frac{i}{\omega_{0}} \partial_{\tau}\right)^{-1} \triangle_{\perp} A \\
& +i \frac{\beta_{0} c \epsilon_{0}}{2} n_{2}\left(1+\frac{i}{\omega_{0}} \partial_{\tau}\right)|A|^{2} A .
\end{aligned}
$$

Der erste Summand auf der rechten Seite von Gleichung (1.51) beschreibt dabei den linearen Anteil der Absorption, deren Stärke durch $\alpha_{0}$ gegeben ist. Der Einfluss der Dispersion sowie der Absorption höherer Ordnung wird durch den zweiten Term dargestellt. Wie in Abschnitt (1.2) gezeigt, stellt die Berücksichtigung der Dispersion bis zur zweiten Ordnung für die für diese Arbeit relevanten Wellenlängen und Wasser als betrachtetem Medium bereits eine sehr gute Näherung dar. Ferner können die Absorptionsterme höherer Ordnung vernachlässigt werden, so dass im Folgenden

$$
\hat{D}=-\frac{\beta_{2}}{2} \partial_{\tau}^{2}
$$

gesetzt wird. Der Term proportional zu $\triangle_{\perp} A$ in Gleichung (1.51) beschreibt die Beugung. Der letzte Summand berücksichtigt die Nichtlinearität des Materials.

Die Einhüllendengleichung entspricht in der dargestellten Form (1.51) Gleichung (6) in [10 mit dem Unterschied, dass sich durch eine andere Definition von $I$ in [10] als (1.32) der Vorfaktor des nichtlinearen Terms unterscheidet.

Oft werden in Gleichung (1.51) die zu $\partial_{\tau}$ proportionalen Anteile vernachlässigt. In diesem Fall wird mit Gleichung (1.52) die Einhüllendengleichung zu einer nichtlinearen Schrödingergleichung. 


\section{Kapitel 2}

\section{Berechnung der Pulsausbreitung}

Die im vorangegangenen Kapitel hergeleitete Einhüllendengleichung stellt im Rahmen dieser Arbeit die theoretische Grundlage für die Berechnung der Ausbreitung ultrakurzer Pulse dar. In diesem Kapitel wird das Verfahren vorgestellt, mit dem diese Gleichung gelöst werden kann. Ferner werden anhand von Beispielen verschiedene Effekte, die bei der Propagation ultrakurzer Pulse - in zunächst räumlich nicht ausgedehnten Systemen - auftreten, diskutiert und Vergleichsrechnungen mit einem auf der direkten Lösung der MaxwellGleichungen basierenden Verfahren vorgestellt.

\subsection{Split-Operator-Verfahren}

Zur numerischen Lösung der Einhüllendengleichung (1.51) wird ein SplitOperator-Verfahren angewendet [1, 32, 37]. Dazu wird diese wie folgt geschrieben:

$$
\partial_{z} A=(\hat{L}+\hat{N}) A
$$

Die Operatoren $\hat{L}$ und $\hat{N}$ fassen die linearen sowie die nichtlinearen Anteile der Ausbreitungsgleichung zusammen. $\hat{L}$ ergibt sich demnach zu:

$$
\hat{L}=\underbrace{-\frac{\alpha_{0}}{2}+i \hat{D}}_{=: \hat{L}_{D}}+\underbrace{\frac{i}{2 \beta_{0}}\left(1+\frac{i}{\omega_{0}} \partial_{\tau}\right)^{-1} \triangle_{\perp}}_{=: \hat{L}_{\perp}} .
$$

Bei der Bestimmung von $\hat{N}$ muss beachtet werden, dass die im nichtlinearen Teil von Gleichung (1.51) auftretende zeitliche Ableitung sich auf das Produkt 
$|A|^{2} A$ bezieht. Mit $|A|^{2}=A^{*} A$ erhält man:

$$
\begin{aligned}
\hat{N} A & =i \frac{\beta_{0} c \epsilon_{0}}{2} n_{2}\left(1+\frac{i}{\omega_{0}} \partial_{\tau}\right)|A|^{2} A \\
& =i \frac{\beta_{0} c \epsilon_{0}}{2} n_{2}\left(|A|^{2} A+\frac{i}{\omega_{0}} \partial_{\tau}|A|^{2} A+\frac{i}{\omega_{0}} A^{*} A \partial_{\tau} A\right) \\
& =i \underbrace{\frac{\beta_{0} c \epsilon_{0}}{2} n_{2}}_{=: q}\left(|A|^{2}+\frac{i}{\omega_{0}}\left(\partial_{\tau}|A|^{2}+A^{*} \partial_{\tau} A\right)\right) A .
\end{aligned}
$$

Durch Integration von Gleichung (2.1) ergibt sich für den Schritt von $z$ zu $z+h$ 1]:

$$
A(z+h)=\exp \left(h \hat{L}+\int_{z}^{z+h} \hat{N}\left(A\left(z^{\prime}\right)\right) d z^{\prime}\right) A(z) .
$$

Für numerische Berechnungen kann das auftretende Integral auf unterschiedliche Weise behandelt werden. Oft wird - wie im Folgenden $-\hat{N}$ in dem betrachteten Intervall $[z, z+h]$ als konstant angenommen und das Integral reduziert sich zu $h \hat{N}(z)$. Alternativ kann eine Näherungsformel wie die Trapezoder Simpson-Formel, die mehrere Stützstellen benötigen, verwendet werden. In diesem Fall muss $\hat{N}$ an den Stützstellen allerdings durch ein iteratives Verfahren bestimmt werden. In jedem Fall ist die resultierende Näherungsformel in niedrigster Ordnung linear von $h$ abhängig.

Die Summe der Operatoren $\hat{L}$ und $\hat{N}$ kann nicht in einem Schritt ausgeführt werden, so dass diese nacheinander angewendet werden müssen:

$$
A(z+h) \approx \exp (h \hat{L}) \exp (h \hat{N}) A(z)
$$

Da $\hat{N}$ und $\hat{L}$ aber im Allgemeinen nicht kommutieren, ist dieses Vorgehen mit einem Fehler behaftet. Nach der Baker-Campbell-Hausdorff-Formel [1]

$$
\exp (\hat{a}) \exp (\hat{b})=\exp \left(\hat{a}+\hat{b}+\frac{1}{2}[\hat{a}, \hat{b}]+\frac{1}{12}[\hat{a}-\hat{b},[\hat{a}, \hat{b}]]+\cdots\right)
$$

ist dieser von der Ordnung $h^{2}[\hat{L}, \hat{N}]$. Die Ordnung kann erhöht werden, wenn man das Verfahren symmetrisch macht:

$$
A(z+h) \approx \exp \left(\frac{h}{2} \hat{L}\right) \exp \left(h \hat{N}\left(z+\frac{h}{2}\right)\right) \exp \left(\frac{h}{2} \hat{L}\right) A(z) .
$$

Wendet man nun die Baker-Campbell-Hausdorff-Formel zweimal auf diesen Ausdruck an, so heben sich die Terme proportional zu $h^{2}$ gerade auf und man hat erreicht, dass der auftretende Fehler nur noch in dritter Ordnung von $h$ abhängt. 
Die in $\hat{L}$ auftretende räumliche Ableitung wird unabhängig von den zeitlichen Ableitungen gelöst (s. Abschnitt 2.2). Mit den in Gleichung (2.2) eingeführten Bezeichnungen ergibt sich:

$A(z+h) \approx \exp \left(\frac{h}{2} \hat{L}_{D}\right) \exp \left(\frac{h}{2} \hat{L}_{\perp}\right) \exp (h \hat{N}) \exp \left(\frac{h}{2} \hat{L}_{\perp}\right) \exp \left(\frac{h}{2} \hat{L}_{D}\right) A(z)$.

\subsection{Berechnung der auftretenden Terme}

Die Berechnung von $\exp \left(h \hat{L}_{D}\right)$ kann für Dispersion beliebiger Ordnung effizient im Fourierraum durchgeführt werden [1]:

$$
\exp (h \hat{L}) A(z)=\mathcal{F}^{-1}\{\exp (h \hat{L}(i \omega)) \mathcal{F}\{A(z)\}\}
$$

$\hat{L}_{D}(i \omega)$ erhält man dabei durch Ersetzen der partiellen Ableitungen $\partial^{n} / \partial t^{n}$ in $\hat{L}_{D}$ durch $(i \omega)^{n}$. Wie man sieht, ist damit für die Berechnung von $\exp \left(h \hat{L}_{D}\right)$ lediglich die Durchführung einer Fouriertransformation sowie einer Rücktransformation nötig, die effizient mit Hilfe eines FFT-Algorithmus durchgeführt werden kann [61]. Bei diesem Vorgehen wird die vorgestellte Methode in der Literatur oft als Split-Step-Fourier-Methode bezeichnet [1].

Auch die Berechnung der transversalen Ableitungen in $\hat{L}_{\perp}$ kann durch Übergang in den Frequenzraum durchgeführt werden. In der vorliegenden Arbeit wurde allerdings das Crank-Nicolson-Verfahren auf Basis von finiten Differenzen gewählt (s. Abschnitt 3.3.3), da es auch die Berechnung der Ableitungen in Zylinderkoordinaten erlaubt.

Für die Berechnung von $\exp (h \hat{N})$ ist im Wesentlichen nur die Bestimmung der zeitlichen Ableitungen von $|A|^{2}$ und $A$ nötig (vgl. Gleichung (2.3) ), diese erfolgt wiederum im Frequenzraum.

\subsection{Schrittweitensteuerung und Diskretisierung}

Um den in Abschnitt 2.1 diskutierten Fehler des Split-Operator-Verfahrens zu kontrollieren, wird üblicherweise eine Schrittweitensteuerung verwendet, die die Größe des Schritts $h$ anpasst. Dabei wird meistens davon ausgegangen, dass der nichtlineare Term (2.3) - und speziell dessen Anteil proportional zu $|A|^{2}$ - dominiert [72]. Dieser bewirkt für jeden Propagationsschritt eine maximale Phasendrehung von $\phi_{\mathrm{NL}}=q \max \left(|A|^{2}\right) h$ (vgl. Abschnitt 2.4 $)$. Dabei bezeichnet $\max \left(|A|^{2}\right)$ das Maximum des Betragsquadrates der Einhüllenden des Pulses bezüglich der Zeit und ggf. der räumlichen Ausdehnung in transversaler Richtung an der Stelle $z$. Fordert man, dass diese Phasendrehung $\phi_{\mathrm{NL}}$ 
einen festgelegten Wert $\phi_{\mathrm{NL}}^{\max }$ nicht überschreiten darf, erhält man als Bedingung für die maximale Schrittweite:

$$
h \leq \frac{\phi_{\mathrm{NL}}^{\max }}{q|A|^{2}} .
$$

In dieser Arbeit wurde jedoch ein anderes Verfahren verwendet [72], das sich universeller einsetzen lässt und insbesondere auch für Regime, in denen die nichtlinearen Effekte nicht dominieren, gut anwendbar ist. Die Schrittweite wird dabei auf Grundlage des relativen lokalen Fehlers zwischen zwei Lösungen unterschiedlicher Genauigkeit bestimmt. Dieses Vorgehen wird in ähnlicher Form oft bei der Lösung gewöhnlicher Differentialgleichungen (zum Beispiel mit dem Runge-Kutta-Verfahren [61]) angewendet. Dazu wird die gesuchte Lösung einmal direkt durch die Durchführung eines Schrittes der Weite $2 h$ berechnet $\left(A_{\mathrm{c}}\right)$, ferner wird eine zweite Lösung $A_{\mathrm{f}}$ durch das nacheinander Ausführen von zwei Schritten mit Schrittweite $h$ bestimmt. Der relative Fehler zwischen diesen beiden Lösungen ergibt sich dann als:

$$
\delta=\frac{\left\|A_{\mathrm{f}}-A_{\mathrm{c}}\right\|}{\left\|A_{\mathrm{f}}\right\|} .
$$

Die auftretende Norm $\|A\|$ ist dabei wie folgt definiert:

$$
\|A(z)\|=\left[\iiint d t d x d y|A(x, y, z, t)|^{2}\right]^{\frac{1}{2}} .
$$

Die Schrittweite wird während der Rechnung so angepasst, dass der auftretende Fehler $\delta$ stets in der Größenordnung eines vorher festgelegten Fehlers $\delta_{0}$ liegt. Ergibt sich während der Rechnung ein Fehler größer $2 \delta_{0}$, wird die Berechnung mit halbierter Schrittweite solange wiederholt, bis der Fehler unterhalb dieser Grenze liegt. Ergebnisse mit einem Fehler kleiner (gleich) $2 \delta_{0}$ werden akzeptiert; für $\delta_{0}<\delta \leq 2 \delta_{0}$ oder $\delta<1 / 2 \delta_{0}$ wird die Schrittweite für die nächste Berechnung um den Faktor $2^{1 / 3}$ erniedrigt bzw. erhöht. Dieser Faktor ergibt sich, wenn man annimmt, dass der Fehler des symmetrischen Split-Operator-Verfahrens in dritter Ordnung von $h$ abhängt (vgl. Abschnitt 2.11).

Zusätzlich zu der beschriebenen Schrittweitensteuerung kann die Berechnung der beiden Lösungen $A_{\mathrm{f}}$ und $A_{\mathrm{c}}$ dazu verwendet werden, die Ordnung des SplitOperator-Verfahrens weiter zu erhöhen. Bezeichnet $A_{t}$ die unbekannte exakte Lösung, dann existiert eine Konstante $\kappa$, so dass gilt:

$$
\begin{aligned}
& A_{\mathrm{c}}=A_{t}+\kappa(2 h)^{3}+O\left(h^{4}\right), \\
& A_{\mathrm{f}}=A_{t}+2 \kappa h^{3}+O\left(h^{4}\right) .
\end{aligned}
$$

Man erhält also eine Lösung 4. Ordnung durch die folgende Linearkombination der beiden Lösungen $A_{\mathrm{f}}$ und $A_{\mathrm{c}}$ [72]:

$$
A_{4}=\frac{4}{3} A_{\mathrm{f}}-\frac{1}{3} A_{\mathrm{c}}=A_{t}+O\left(h^{4}\right) .
$$


Dabei muss man allerdings beachten, dass dieser Überlegung die Annahme zugrunde liegt, dass der Fehler des Split-Operator-Verfahrens der dominierende ist. Insbesondere der Fehler durch die recht grobe Integration des nichtlinearen Anteils (vgl. Abschnitt 2.1) und die Verwendung des Crank-NicolsonVerfahrens (vgl. Abschnitt 3.3.3) kann u. U. den Split-Operator-Fehler überwiegen. In diesem Fall ist eine Berechnung nach Gleichung (2.10) nicht sinnvoll. Die Schrittweitensteuerung nach dem beschriebenen Verfahren kann aber trotzdem angewendet werden, da sie - im Gegensatz zum Verfahren nach Gleichung (2.9) - den Gesamtfehler kontrolliert.

Neben der Wahl der Schrittweite in z-Richtung muss bei der Anwendung der Split-Fourier-Methode besonders auf die Wahl des bei der Berechnung verwendeten Bereichs für den zeitlichen Verlauf des Pulses geachtet werden. Dabei sollte die Größe des zeitlichen Fensters typischerweise zehn bis zwanzig Mal größer als die Pulsbreite gewählt werden [1]. Dies ist nötig, da bei der Berechnung der Fouriertransformation periodische Randbedingungen impliziert werden, und demnach gewährleistet sein muss, dass der Puls am Rand ausreichend abgeklungen ist, um Fehler bei Berechnungen am Rand zu vermeiden. Die Weite der Diskretisierung des gewählten Bereichs ist nicht so kritisch und kann in der Regel relativ grob erfolgen (z. B. 1024 Punkte), wodurch eine sehr schnelle Durchführung der Fouriertransformation ermöglicht wird.

Die gegebenenfalls nötige Diskretisierung in transversaler Richtung wird in Abschnitt 3.3.2 diskutiert.

\subsection{Beispiele}

Als erstes Beispiel wird die Berechnung eines sog. few-cycle-Pulses vorgestellt. Dabei handelt es sich um einen Puls, der nur wenige Perioden der mittleren Frequenz enthält. Bei so kurzen Pulsen ist die direkte Berechnung über die Maxwell-Gleichungen mit der Finite-Difference-Time-DomainMethode (FDTD) [34, 40, 38] möglich und wurde zum Vergleich durchgeführt. Damit eignen sich diese Pulse gut zum Test des Simulationsprogramms und ferner, um die wesentlichen Effekte der Pulsausbreitung darzustellen.

Analog zu den Berechnungen von Brabec und Krausz 10 wird ein Puls mit einer Wellenlänge von $800 \mathrm{~nm}$ betrachtet, der zu Beginn ein sech-Profil mit $|A(z=0, \tau=0)|^{2}=4 \cdot 10^{13} \mathrm{~W} / \mathrm{cm}^{2}$ und $\tau_{\mathrm{FWHM}}=2.67 \mathrm{fs}$ besitzt. Die optischen Eigenschaften des Mediums sind Quarzglas angenähert:

$$
\begin{array}{ll}
\alpha_{0}=0 & \text { Absorption wird vernachlässigt } \\
n_{0}=1.45 & \text { linearer Brechungsindex } \\
n_{2}=3 \cdot 10^{-16} \frac{\mathrm{cm}^{2}}{\mathrm{~W}} & \text { nichtlinearer Brechungsindex } \\
\omega_{0}=2.35 \mathrm{fs}^{-1} & \text { mittlere Frequenz } \\
d n /\left.d \omega\right|_{\omega_{0}}=5.776 \cdot 10^{-3} \text { fs } & \text { Dispersion 1. Ordnung } \\
d^{2} n /\left.d \omega^{2}\right|_{\omega_{0}}=0 & \text { Dispersion 2. Ordnung }
\end{array}
$$




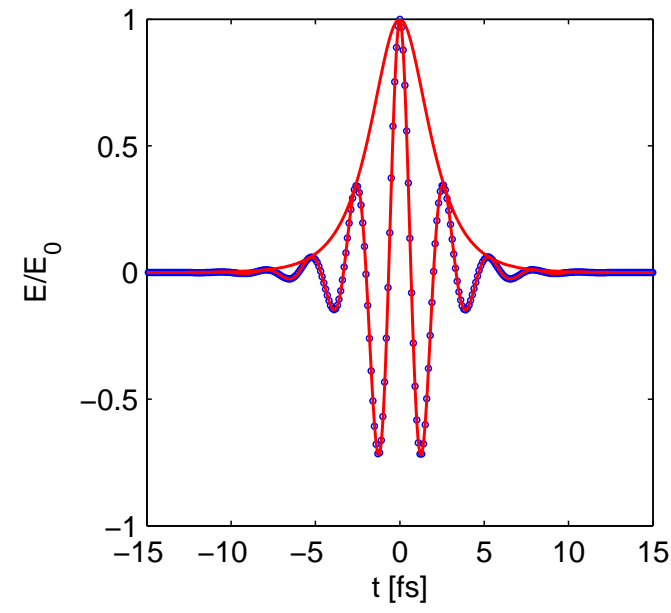

(a) Beginn der Simulation, $\mathrm{z}=0 \mu \mathrm{m}$

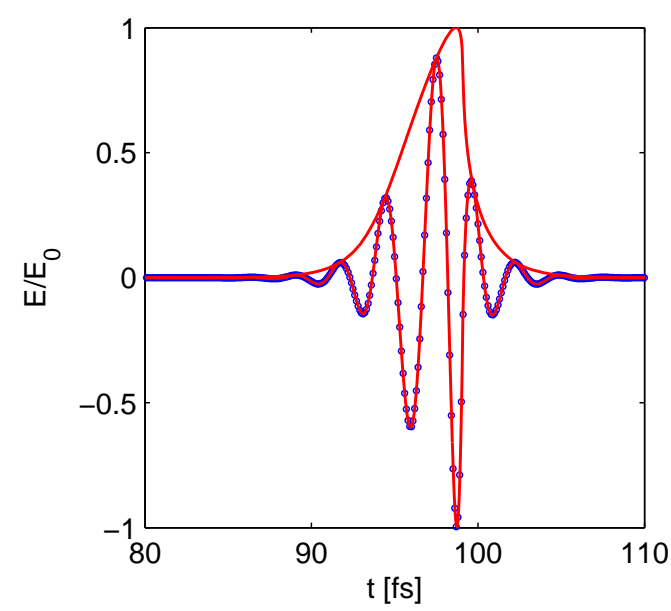

(c) ohne Dispersion $(d n / d \omega=0)$, $\mathrm{z}=20 \mu \mathrm{m}$

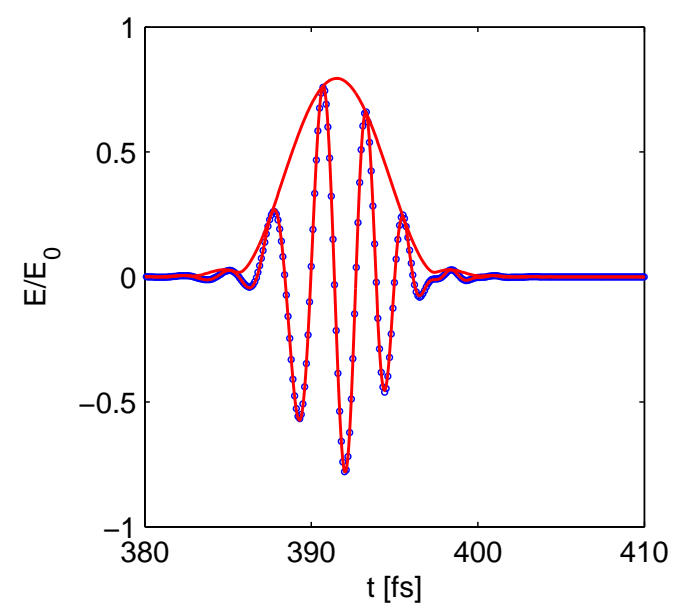

(b) ohne Nichtlinearität $\left(n_{2}=0\right)$

$$
\mathrm{z}=80 \mu \mathrm{m}
$$

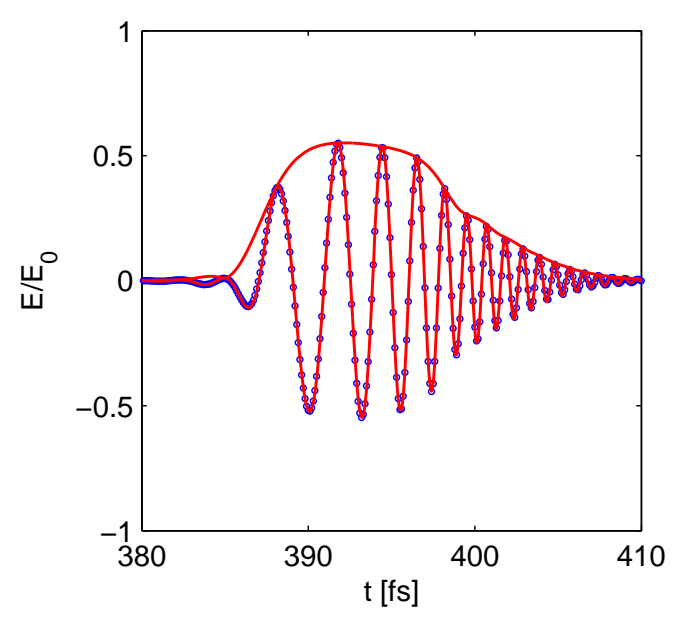

(d) mit Dispersion und Nichtlinearität, $\mathrm{z}=80 \mu \mathrm{m}$

Abbildung 2.1: Ergebnisse der Simulation der Ausbreitung eines fewcycle-Pulses [10] in einem nichtlinearen dispersiven Medium. Die durchgezogenen roten Kurven stellen das mit Hilfe der Einhüllendengleichung berechnete elektrische Feld und dessen Einhüllende dar. Zum Vergleich ist das Ergebnis der direkten Berechnung über die Maxwell-Gleichungen als blau gepunktete Kurve dargestellt.

Die in der Simulation verwendeten Parameter $\beta_{0}, \beta_{1}$ und $\beta_{2}$ (vgl. Gleichung (1.39) ) können aus den Werten für die Dispersion über die entsprechenden Ableitungen von $k(\omega)=\omega \cdot n(\omega) / c$ nach (1.11) und (1.12) bestimmt werden, es ergibt sich insbesondere $\beta_{2}=\mathrm{GVD} \approx 38.53 \mathrm{fs}^{2} / \mathrm{mm}$.

Abbildung 2.1 zeigt die mit Hilfe der Einhüllendengleichung (rote Linie) und den Maxwell-Gleichungen (blaue Punkte) berechneten Pulsformen. Man sieht sofort, dass sich eine sehr gute Übereinstimmung zwischen den mit beiden Me- 
thoden berechneten Kurven ergibt. Allerdings muss dabei beachtet werden, dass für die Berechnung über die Maxwell-Gleichung die gleichen Näherungen für die Nichtlinearität und die Dispersion wie bei der Einhüllendengleichung gemacht wurden.

Im Falle der Nichtlinearität ist dafür nach den Gleichungen (1.18) und (1.32) allerdings die Bestimmung der Einhüllenden des elektrischen Feldes notwendig, die bei einem rein reellen Ansatz nicht möglich ist. Für die in Abbildung 2.1 gezeigten Vergleichsrechnungen wurde daher auch für die Berechnung über die Maxwell-Gleichungen ein komplexwertiger Ansatz gewählt.

Üblicherweise wird für die Berechnung des linearen Anteils der Polarisation bei der Berechnung über die Maxwell-Gleichungen ein Oszillatormodell für die Polarisation zugrunde gelegt [38. Durch Anpassen des Finite-DifferenzenSchemas kann der Einfluss der Polarisation jedoch auch analog zum Vorgehen in Abschnitt 1.5.2 bestimmt werden. Man muss dabei allerdings beachten, dass - neben der Linearisierung - bei der Bestimmung der Dispersionseigenschaften über die Multiplikation mit der Polarisation im Spektralbereich das Einschwingen der Oszillatoren vernachlässigt wird.

Neben dem Vergleich der beiden Propagations-Methoden ermöglichen die Abbildungen 2.1, die Auswirkungen der Dispersion und der Nichtlinearität auf die Pulsausbreitung zu beobachten.

Vergleicht man die Pulsform für den nur der Dispersion ausgesetzten Puls (Abbildung 2.1 (b)) mit dem Ausgangspuls (Abbildung 2.1 (a)), kann man erkennen, dass sich der Puls unter dem Einfluss der Gruppengeschwindigkeitsdispersion verbreitert hat. Ferner sieht man, dass sich neben der Breite auch die Form der Einhüllenden leicht verändert hat, was insbesondere an den entstandenen Nebenmaxima der Einhüllenden an den beiden Seiten des Pulses zu sehen ist. Dies tritt dann auf, wenn das zeitliche Profil des Pulses - wie im vorliegenden Fall das sech-Profil - von der Gauß-Form abweicht. Neben der Veränderung der Pulsform kann man in Abbildung 2.1 (b) auch erkennen, dass sich die Frequenz des elektrischen Feldes mit der Zeit verändert. Dieses Phänomen wird als Chirp bezeichnet. Im Falle einer normalen Gruppengeschwindigkeitsdispersion (GVD >0) ist dieser linear und positiv, d. h. die Frequenz steigt im zeitlichen Verlauf linear an. Ein Puls mit Chirp besitzt (unabhängig von dessen Richtung) ein breiteres Spektrum als ein Puls gleicher Länge ohne Chirp, dessen Spektrum (für die entsprechende Pulsform) eine minimale Breite besitzt; man spricht in diesem Fall von einem transformlimitierten Puls.

Betrachtet man die Wirkung der Nichtlinearität auf den Puls (Abbildung 2.1 (c)), fällt zunächst die Selbstaufsteilung des Pulses auf. Diese wird durch den Term proportional zu $\partial_{t}|A|^{2} A$ verursacht und spielt nur bei Pulsen von wenigen Femtosekunden Länge und vernachlässigbarer GVD eine Rolle. Bei genauer Betrachtung des Verlaufs des elektrischen Feldes kann man erkennen, dass sich die Frequenz auch unter dem Einfluss der Nichtlinearität ändert, allerdings ist 
die Änderung in diesem Fall nichtlinear und abhängig von der Pulsform. Dieser Effekt wird Selbstphasenmodulation genannt. Abbildung 2.2 verdeutlicht den Einfluss der Selbstphasenmodulation durch die Wahl eines etwas längeren Pulses und der Vernachlässigung des für die Selbstaufsteilung verantwortlichen Terms der Ausbreitungsgleichung. Man erkennt deutlich die Ausbildung des Chirps, für den bei einem sech-förmigen Puls gilt (vgl. Abschnitt 2.3, [1]):

$$
\Delta \omega=-\frac{\partial}{\partial t} \phi_{\mathrm{NL}}=q z A_{0}^{2} \frac{\partial}{\partial t} \operatorname{sech}^{2}\left(\frac{t}{t_{0}}\right)=-\frac{2 q z A_{0}^{2}}{t_{0}} \operatorname{sech}^{2}\left(\frac{t}{t_{0}}\right) \tanh \left(\frac{t}{t_{0}}\right) .
$$
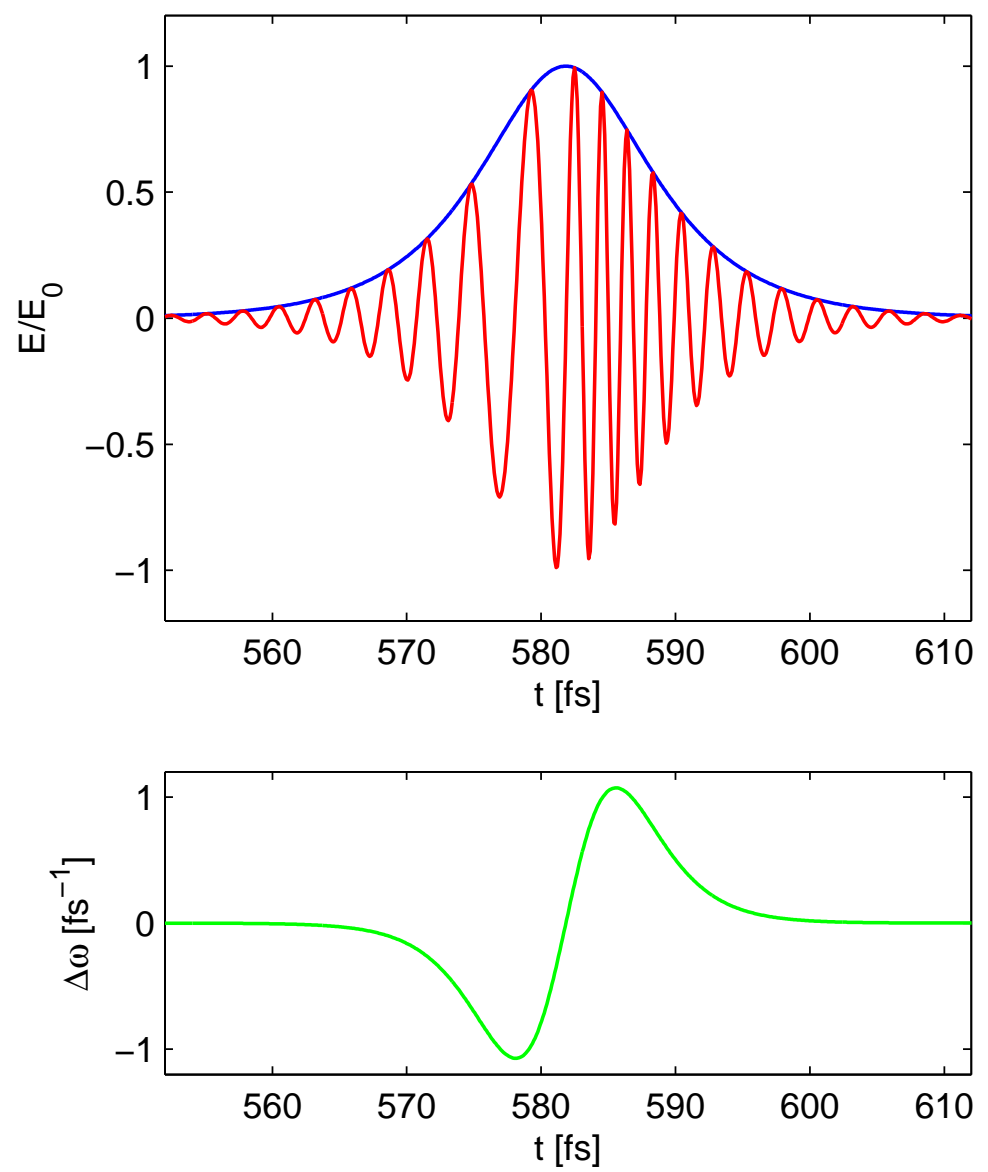

Abbildung 2.2: Selbstphasenmodulation bei einem sech-förmigen Puls mit FWHM $=10$ fs nach $120 \mu \mathrm{m}$ Propagation durch ein nichtlineares Medium (Eigenschaften wie Abb. 2.1, Rechnung ohne Dispersion und Selbstaufsteilung). Man erkennt, dass die Einhüllende (blau) des elektrischen Feldes (rot) seine Form nicht verändert. Die grüne Kurve zeigt den durch die Selbstphasenmodulation induzierten Chirp $\Delta \omega$ des Pulses.

Abbildung 2.1 (d) zeigt den Puls unter Einwirkung der Dispersion und der Nichtlinearität. Man erkennt, dass der Puls im Vergleich zur Propagation durch ein rein dispersives Medium deutlich verbreitert ist. Dies geht einher mit einem 
stark ausgeprägten Chirp, der durch die Wechselwirkung der (normalen) Gruppengeschwindigkeitsdispersion mit der Nichtlinearität verursacht wird und zu einer sehr starken Verbreiterung des Pulsspektrums führen kann (white light continuum generation, [76]).

Betrachtet man einen Puls in einem Medium mit GVD $<0$ (anormale Gruppengeschwindigkeitsdispersion), so kann man (bei vernachlässigbarer Selbstaufsteilung und geeigneter Wahl der Intensität) erreichen, dass sich die Wirkung der beiden Effekte aufhebt und sich der Puls während der Propagation nicht verändert. Diese Pulse nennt man Solitonen [1]. Für höhere Intensitäten existieren auch Solitonen höherer Ordnung, deren Pulsform sich periodisch entwickelt (s. Abbildung 2.3 (b)).

(a)

(b)
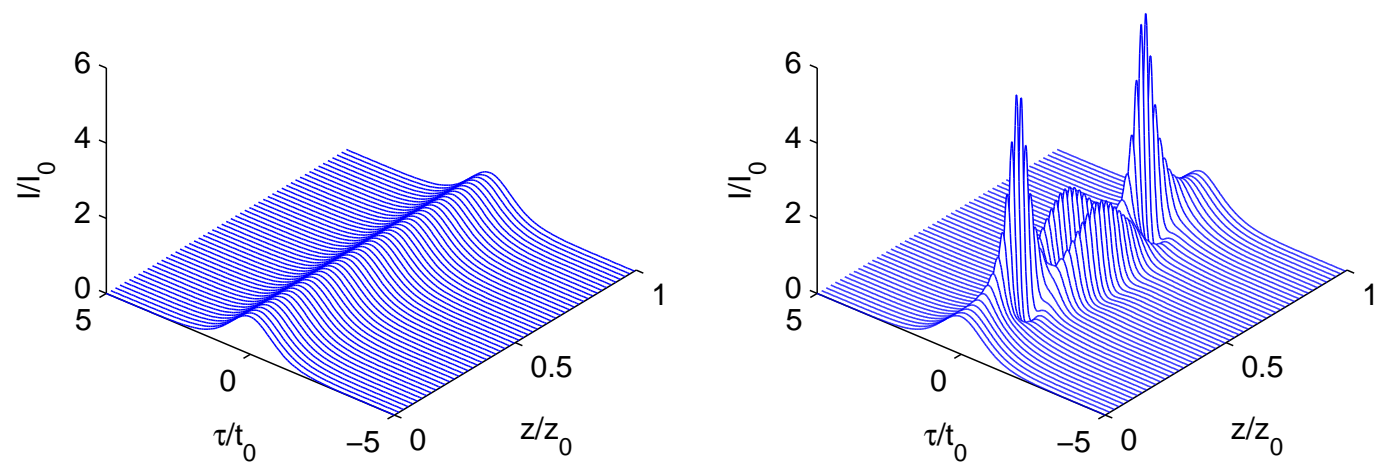

Abbildung 2.3: Propagation von Solitonen erster (a) und dritter Ordnung (b), Parameter nach Agrawal [1]. 


\section{Kapitel 3}

\section{Pulsausbreitung in räumlich ausgedehnten Systemen}

In diesem Kapitel steht bei der Ausbreitung elektromagnetischer Wellen der Einfluss ihrer räumlichen Ausdehnung (senkrecht zur Ausbreitungsrichtung) im Vordergrund. Dabei wird zunächst der Fall eines Lasers im Dauerbetrieb (continuous wave) betrachtet, also die Ausbreitung eines Lichtstrahls unendlicher zeitlicher Dauer. Die gewonnenen Erkenntnisse werden dann auf die Berechnung der Propagation von Laserpulsen im Raum angewendet.

Im Vordergrund steht dabei die Berechnung von Laserpulsen beim Durchlaufen des Fokusbereichs. Es wird die Abhängigkeit der Fokussierbarkeit vom Strahlprofil ebenso diskutiert wie der Einfluss der Nichtlinearitäten auf das Verhalten des Laserpulses im Fokus (Selbstfokussierung). Als Beispiel wird ein System vorgestellt, bei dem sich der Puls nach der Fokussierung in zwei Teile aufteilt (Pulse Splitting).

\subsection{Paraxiale Wellengleichung}

Zunächst wird die Ausbreitung von ungepulstem Laserlicht in einem linearen Medium betrachtet, das zur Vereinfachung als nicht absorbierend angenommen wird. In diesem Fall können alle zeitlichen Abhängigkeiten in der Ausbreitungsgleichung (1.51) vernachlässigt werden und man erhält die paraxiale Wellengleichung:

$$
\partial_{z} A=\frac{i}{2 \beta_{0}} \triangle_{\perp} A
$$

In der Literatur findet man diese Gleichung je nach Ansatz der Einhüllenden (vgl. Gleichung (1.26)) auch mit negativem Vorzeichen [1]. 


\subsubsection{Gaußsche Strahlen}

Eine besonders einfache Beschreibung von Laserstrahlung wird durch Gaußsche Strahlung gegeben. Diese beschreibt Licht von Lasern, die Strahlung mit einem gaußförmigen Strahlprofil (in der transversalen Grundmode $\mathrm{TEM}_{00}$ ) emittieren.

Gaußsche Strahlen können mathematisch durch [71]

$$
A(r, z)=A_{\mathrm{g}}(z) \exp \left(-\frac{r^{2}}{w^{2}(z)}-i \phi(z)-i \beta_{0} \frac{r^{2}}{2 R(z)}\right)
$$

beschrieben werden. Dabei bezeichnet $w(z)$ den Strahlradius und $R(z)$ die Krümmung. Ferner ist durch $A_{\mathrm{g}}(z)$ die Amplitude und durch $\phi(z)$ die Phase gegeben. Gaußsche Strahlen stellen eine Lösung der paraxialen Wellengleichung dar: Ein Strahl mit einem Gaußschen Strahlprofil wird dieses unter den Bedingungen der paraxialen Näherung (s. a. Abschnitt 1.5.2) immer beibehalten. Daher ist es möglich, die Strahlausbreitung nur durch Bestimmung der in Gleichung (3.2) von z abhängigen Größen zu beschreiben.

So ergibt sich durch Einsetzen von Gleichung (3.2) in die paraxiale Wellengleichung (3.1) für die Ausbreitung eines zu Beginn der Propagation an der Stelle $z=0$ ungekrümmten Gaußschen Strahls $\left(R(0)=\infty, w(0)=w_{0}, A_{\mathrm{g}}(0)=A_{0}\right.$, $\left.\phi(0)=\phi_{0}\right)$ in einem Medium mit Brechungsindex $n_{0}$ [71]:

$$
\begin{aligned}
w(z) & =w_{0} \sqrt{1+\left(\frac{z}{z_{\mathrm{R}}}\right)^{2}}, & R(z) & =z+\frac{z_{\mathrm{R}}^{2}}{z}, \\
A_{\mathrm{g}}(z) & =A_{0} \frac{w_{0}}{w(z)}, & \phi(z) & =\tan ^{-1}\left(\frac{z}{z_{\mathrm{R}}}\right) .
\end{aligned}
$$

Die auftretende Größe $z_{\mathrm{R}}$ wird Rayleigh-Länge genannt. Für sie gilt:

$$
z_{\mathrm{R}}=\frac{\pi w_{0}^{2} n_{0}}{\lambda}
$$

Man erkennt aus der ersten Gleichung von (3.3), dass $z_{\mathrm{R}}$ die Strecke beschreibt, nach der der Strahlradius eines zunächst ebenen Gaußschen Strahls der Breite $w_{0}$ und der Wellenlänge $\lambda$ in einem gegebenen Medium mit Brechungsindex $n$ um den Faktor $\sqrt{2}$ zunimmt.

Außerdem sieht man, dass der Strahlradius für $z \gg z_{\mathrm{R}}$ linear in $z$ zunimmt:

$$
w(z)=w_{0} \sqrt{1+\left(\frac{z}{z_{\mathrm{R}}}\right)^{2}} \approx w_{0} \frac{z}{z_{R}}=\frac{\lambda z}{\pi w_{0} n_{0}} .
$$

Im Folgenden wird ein Gaußscher Strahl betrachtet, der durch eine Linse mit der Brennweite $f$ fokussiert wird. Zunächst wird angenommen, dass der 


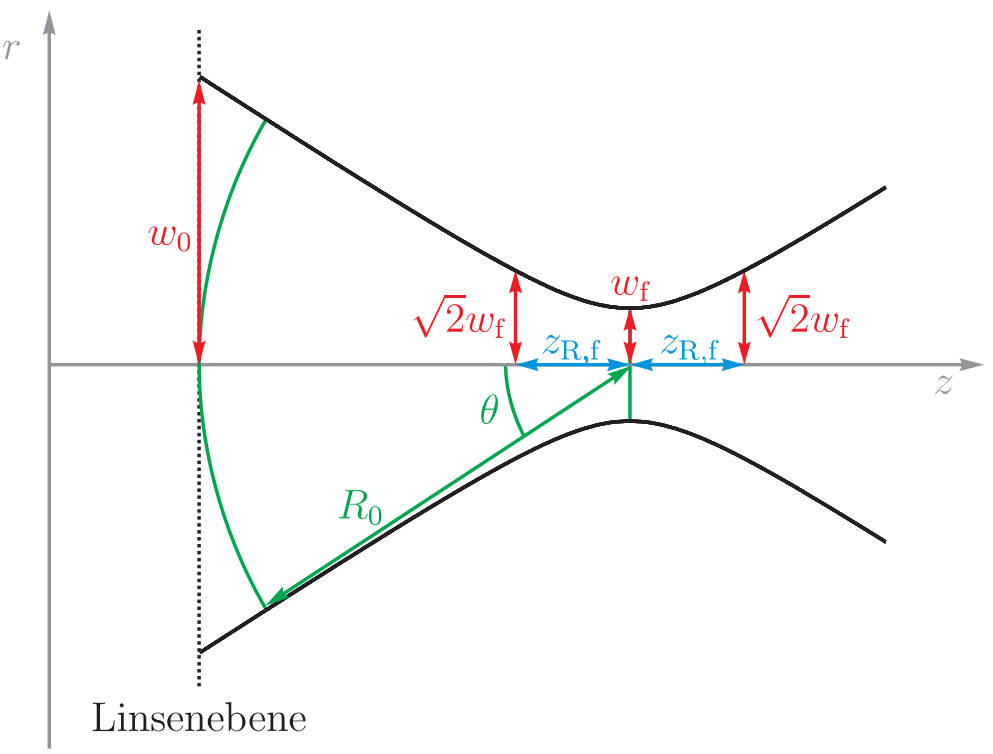

Abbildung 3.1: Schematische Darstellung der Fokussierung eines Gaußstrahls

Strahl im Brennpunkt der Linse $\left(b_{\mathrm{f}}=f\right)$ keine Krümmung aufweist. In diesem Fall kann die Ausbreitung ebenfalls durch die Gleichungen (3.3) beschrieben werden, wenn die Bezeichnungen entsprechend angepasst werden: $w_{\mathrm{f}} \rightarrow w_{0}$, $z_{\mathrm{R}, \mathrm{f}} \rightarrow z_{\mathrm{R}}$ (vgl. Abbildung 3.1) .

Insbesondere ergibt sich mit der Näherung (3.5) für den Strahlradius im Fokus (die sogenannte Strahltaille):

$$
w_{\mathrm{f}}=\frac{\lambda f}{\pi w_{0} n_{0}} \approx \frac{\lambda}{\pi n_{0} \theta} .
$$

Dabei bezeichnet $\theta$ den (halben) Divergenzwinkel (vgl. Abbildung 3.1). Für die Rayleigh-Länge des fokussierten Strahls $z_{\mathrm{R}, \mathrm{f}}$ findet man als Funktion der Brennweite der Linse $f$ und des Strahlradius in der Linsenebene $w_{0}$ :

$$
z_{\mathrm{R}, \mathrm{f}}=\frac{\pi w_{\mathrm{f}}^{2} n_{0}}{\lambda}=\frac{\lambda f^{2}}{\pi w_{0}^{2} n_{0}}
$$

Fordert man, dass der Strahl statt im Fokus in der Linsenebene ungekrümmt ist, so verschiebt sich der Brennpunkt $b_{\mathrm{f}}$ und man erhält eine etwas kleinere Strahltaille $w_{\mathrm{f}}$ :

$$
b_{\mathrm{f}}=\frac{f}{1+\left(\frac{\lambda f}{\pi w_{0}^{2} n_{0}}\right)^{2}}, \quad w_{\mathrm{f}}=\frac{\lambda f}{\pi w_{0} n_{0} \sqrt{1+\left(\frac{\lambda f}{\pi w_{0}^{2} n_{0}}\right)^{2}}} .
$$




\subsubsection{Höhere transversale Lasermoden}

Die Gaußschen Strahlen (3.2) stellen die Lösung niedrigster Ordnung für die paraxiale Wellengleichung dar. Darüber hinaus existieren Lösungen höherer Ordnung, die Gauß-Hermite- und die Gauß-Laguerre-Funktionen. Die Gauß-Hermite-Funktionen $A_{n m}$ sind in kartesischen und die Gauß-LaguerreFunktionen $A_{p m}$ in zylindrischen Koordinaten formuliert. Beide Eigenfunktionssysteme bilden eine Basis für beliebige Lösungen der paraxialen Wellengleichung.

Reale Laser zeigen in der Regel kein reines Gauß-Profil. Dies ist zum Beispiel der Fall, wenn höhere transversale Moden im Resonator anschwingen.

Um Pulse aus solchen Lasern untersuchen zu können, werden in der vorliegenden Arbeit auch nicht-Gaußsche Pulse betrachtet. Dabei werden überwiegend rotationssymmetrische Pulse untersucht, bei denen die Gauß-LaguerreFunktionen Anwendung finden. Es ist allerdings anzumerken, dass sich Abweichungen vom Gaußprofil nicht auf rotationssymmetrische Profile beschränken. Weitere Ursachen für Abweichungen vom Gauß-Profil kann eine inhomogene Verstärkung im Lasermedium und die Ausbildung von Teilstrahlen sein [25]. Ferner kann zum Beispiel durch gekippte Bauteile wie Brewster-Fenster die Rotationssymmetrie aufgehoben werden.

Für die zylindersymmetrischen Gauß-Laguerre-Funktionen $A_{p 0}$ findet man [71] (vgl. Abbildung [3.2):

$$
A_{p 0}(r, z) \sim \mathrm{L}_{p}\left(\frac{2 r^{2}}{w^{2}(z)}\right) \exp \left(-\frac{r^{2}}{w^{2}(z)}-i \beta_{0} \frac{r^{2}}{2 R}\right) .
$$

$\mathrm{L}_{p}$ bezeichnet dabei die sogenannten Laguerreschen Polynome [13], die in den niedrigsten Ordnungen durch

$$
\begin{aligned}
& \mathrm{L}_{0}(x)=1 \\
& \mathrm{~L}_{1}(x)=-x+1 \\
& \mathrm{~L}_{2}(x)=\frac{1}{2}\left(x^{2}-4 x+2\right)
\end{aligned}
$$

gegeben sind.

\subsubsection{Bestimmung des Strahlradius}

Für Gaußsche Strahlen ergibt sich der Strahlradius w nach Gleichung (3.2) über den Abstand der Mitte des Strahls zu dem Punkt, an dem das elektrische Feld auf 1/e des Maximalwertes gesunken ist. Für nicht-Gaußsche Strahlen muss diese Definition erweitert werden. Dabei sind verschiedene Methoden, den Strahlradius festzulegen, denkbar. Häufig wird der transversale Verlauf 


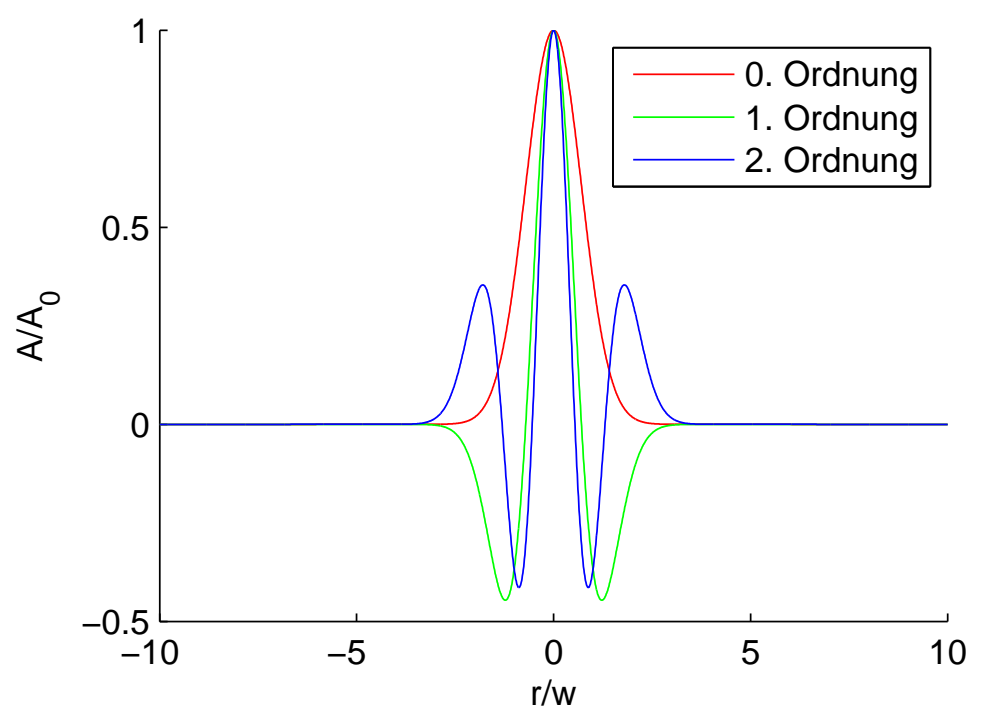

Abbildung 3.2: Radialer Verlauf des elektrischen Feldes von Strahlen mit Gauß-Laguerre-Profil unterschiedlicher Ordnung

der Intensität als Verteilung aufgefasst, und der Laserstrahl über die Momente dieser Verteilung beschrieben [24]. Das erste Moment, der Erwartungswert, beschreibt dabei den Schwerpunkt des Strahls. Der Strahlradius kann über das zweite Moment, die Varianz, bestimmt werden, die in $x$-Richtung durch

$$
\left\langle x^{2}(z)\right\rangle=\frac{\iint(x-\langle x\rangle)^{2} I(x, y, z) d x d y}{\iint I(x, y, z) d x d y}
$$

gegeben ist. Für zylindersymmetrische Strahlen erhält man:

$$
\begin{aligned}
\left\langle x^{2}(z)\right\rangle & =\frac{\int_{0}^{2 \pi} d \theta \cos ^{2}(\theta) \int_{0}^{\infty} d r r^{3} I(r, \theta, z)}{\int_{0}^{2 \pi} d \theta \int_{0}^{\infty} d r r I(r, \theta, z)} \\
& =\frac{\int_{0}^{\infty} d r r^{3} I(r, z)}{2 \int_{0}^{\infty} d r r I(r, z)} .
\end{aligned}
$$

Der Strahlradius $w(z)$ wird nun durch das Zweifache der Standardabweichung definiert 24] und ergibt sich mit Gleichung (3.10) wie folgt:

$$
w(z)=2 \sqrt{\left\langle x^{2}(z)\right\rangle}=\sqrt{\frac{2 \int_{0}^{\infty} d r r^{3} I(r, z)}{\int_{0}^{\infty} d r r I(r, z)}} .
$$

Durch Einsetzen von Gleichung (3.2) in Gleichung (3.11) und Verwendung von Gleichung (1.32) kann man leicht erkennen, dass diese Definition konsistent mit der Definition des Strahlradius für Gaußsche Strahlen ist:

$$
w(z)=\sqrt{\frac{2 \int_{0}^{\infty} d r r^{3} \exp \left(-\frac{2 r^{2}}{w_{\mathrm{g}}^{2}(z)}\right)}{\int_{0}^{\infty} d r r \exp \left(-\frac{2 r^{2}}{w_{\mathrm{g}}^{2}(z)}\right)}}=\sqrt{\frac{2 \frac{1}{8} w_{\mathrm{g}}^{4}(z)}{\frac{1}{4} w_{\mathrm{g}}^{2}(z)}}=w_{\mathrm{g}}(z) .
$$




\subsubsection{Fokussierbarkeit nicht-Gaußscher Strahlen}

Eine wichtige Größe bei der Fokussierung von Laserstrahlung stellt das Strahlparameterprodukt dar. Dieses ergibt sich aus dem Produkt des Divergenzwinkels mit der Strahltaille. Nach Gleichung (3.6) erhält man für einen Gaußschen Strahl:

$$
w_{\mathrm{f}} \theta=\frac{\lambda}{\pi n_{0}} .
$$

Reale Laserstrahlen besitzen in der Regel kein ideales Gauß-Profil. Ein Maß für die Fokussierbarkeit eines solchen Laserstrahls kann durch die Beugungsmaßzahl $M^{2}$ gegeben werden. Manchmal wird auch die Strahlqualität verwendet, die durch

$$
K=\frac{1}{M^{2}}
$$

gegeben ist [24]. Für einen Gauß-Strahl gilt $M^{2}=1$ und $K=1$. Abweichungen von diesem Profil führen zu einer größeren Beugungsmaßzahl $M^{2}>1$ und einer geringeren Strahlqualität $K<1$.

Für reale Laserstrahlen wird das Strahlparameterprodukt (3.12) zu:

$$
w_{\mathrm{f}} \theta=M^{2} \frac{\lambda}{\pi n_{0}} .
$$

Man erkennt also, dass das Produkt aus Fokuswinkel und Breite des Strahls im Fokus für einen Gauß-Strahl minimal ist. Insbesondere führt ein größeres $M^{2}$ bei identischem Fokuswinkel zu einer breiteren Strahltaille (vgl. Abbildung 3.3). ,

Für die in Abschnitt 3.1 .2 beschriebenen Gauß-Laguerre-Moden $A_{p 0}$ ergibt sich für die Beugungsmaßzahl $M^{2}=p^{2}+1$ [24]. Um einen Strahl mit einem bestimmten $M^{2}$ theoretisch zu beschreiben, werden in der Simulation Kombinationen mehrerer Moden verwendet. Dabei muss beachtet werden, dass die Gauss-Laguerre-Moden unterschiedliche Strahlbreiten besitzen. Dies kann durch entsprechende Skalierung allerdings leicht behoben werden.

Experimentell kann die Beugungsmaßzahl und damit die Strahlqualität eines Laserstrahls auf unterschiedliche Art bestimmt werden. Die entsprechende ISO-Norm 11146 beschreibt ein Verfahren, bei dem der zu untersuchende Laserstrahl mit einer Linse fokussiert und die Strahlbreite an verschiedenen Stellen um den Fokusbereich bestimmt wird. Die Messung der Strahlbreite wird dabei mit einer beweglichen Blende oder Schneide durchgeführt [24]. Die Beugungsmaßzahl kann dann durch einen Fit (vgl. Abbildung 3.3) berechnet werden. Alternativ läßt sich die Beugungsmaßzahl direkt durch Analyse des Strahls mit Hilfe eines Hartmann-Shack-Wellenfrontsensors bestimmen [68]. 


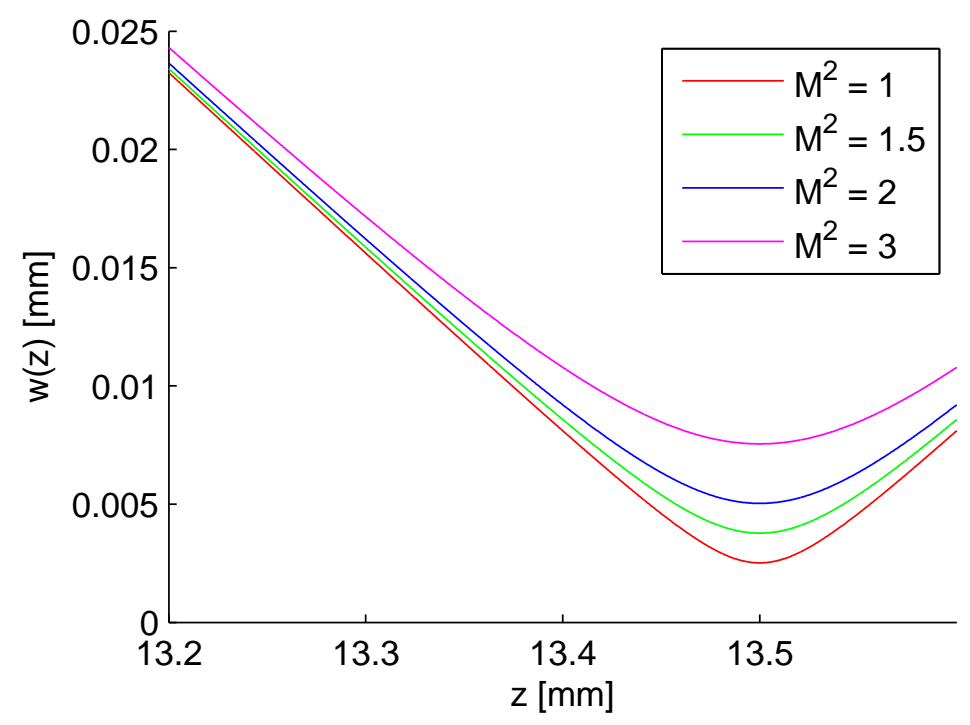

Abbildung 3.3: Verlauf der Breite fokussierter Laserstrahlen unterschiedlicher Strahlqualität. Für alle Strahlen beträgt die Brennweite $13.5 \mathrm{~mm}$, der Fokuswinkel $\sim 4.42^{\circ}$ und die Wellenlänge $810 \mathrm{~nm}$ bei einem Brechungsindex von $n_{0}=1.328$ (vgl. Abschnitt 5.3 ). Für den idealen Gauß-Strahl $\left(M^{2}=1\right)$ wird die Strahltaille mit $w_{\mathrm{f}} \approx 2.52 \mu \mathrm{m}$ minimal. Man beachte, dass die Strahlverläufe gestaucht dargestellt sind.

\subsubsection{Das Kirchhoffsche Beugungsintegral}

Eine zum Lösen einer Differentialgleichung wie der (paraxialen) Wellengleichung alternative Vorgehensweise der Berechnung der Strahlausbreitung stellt die Lösung des Kirchhoffschen Beugungsintegrals dar. Ist das elektrische Feld in einer Ebene $(x, y, z=0)$ bekannt, so kann das Feld an einem Punkt $\left(x^{\prime}, y^{\prime}, z^{\prime}\right)$ nach dem Huygensschen Prinzip durch die Überlagerung von sekundären Kugelwellen, die an jedem Punkt der Ursprungsebene entstehen, bestimmt werden. Mit den Bezeichnungen $\mathbf{r}=\left(x^{\prime}-x, y^{\prime}-y, z^{\prime}\right), \hat{\mathbf{r}}=\mathbf{r} /|\mathbf{r}|$ und $\hat{\mathbf{n}}=(0,0,1)$ gilt [51]:

$$
E\left(x^{\prime}, y^{\prime}, z^{\prime}\right)=\frac{1}{i \lambda} \iint E(x, y) \frac{1}{|\mathbf{r}|} e^{i k|\mathbf{r}|} \cos (\hat{\mathbf{n}}, \hat{\mathbf{r}}) d x d y .
$$

Man kann zeigen, dass das Kirchhoffsche Beugungsintegral in guter Näherung eine Lösung der Maxwell-Gleichungen darstellt [8].

In der paraxialen Näherung kann $\cos (\hat{\mathbf{n}}, \hat{\mathbf{r}}) \approx 1$ angenommen und das in der Exponentialfunktion auftretende $|\mathbf{r}|$ durch eine Taylorentwicklung bis zur zweiten Ordnung in $\sqrt{\left(x^{\prime}-x\right)^{2}+\left(y^{\prime}-y\right)^{2}}$ genähert werden [51]. Man erhält für das Kirchhoffsche Beugungsintegral in paraxialer oder Fresnelscher Näherung:

$$
E\left(x^{\prime}, y^{\prime}, z^{\prime}\right)=\frac{e^{i k z^{\prime}}}{i \lambda z^{\prime}} \iint E(x, y) e^{\frac{i k}{2 z^{\prime}}\left[\left(x^{\prime}-x\right)^{2}+\left(y^{\prime}-y\right)^{2}\right]} d x d y
$$


In Zylinderkoordinaten ausgedrückt wird Gleichung (3.14) zu:

$$
E\left(r^{\prime}, \theta^{\prime}, z^{\prime}\right)=\frac{e^{i k z^{\prime}}}{i \lambda z^{\prime}} \int_{0}^{\infty} d r r \int_{0}^{2 \pi} E(r, \theta) e^{i \frac{\pi}{\lambda z^{\prime}}\left[r^{\prime 2}+r^{2}-2 r^{\prime} r \cos \left(\theta^{\prime}-\theta\right)\right]} d \theta .
$$

Ist das Feld rotationssymmetrisch, so ergibt sich mit der Besselfunktion 0. Ordnung erster Gattung $J_{0}$ [13, 71]:

$$
E\left(r^{\prime}, z^{\prime}\right)=\frac{2 \pi e^{i k z^{\prime}}}{i \lambda z^{\prime}} \int_{0}^{\infty} d r r E(r) e^{-i \frac{\pi}{\lambda z^{\prime}}\left[r^{\prime 2}+r^{2}\right]} J_{0}\left(\frac{2 \pi r^{\prime} r}{\lambda z^{\prime}}\right) .
$$

\subsubsection{Aberrationen}

Bei den bisher beschriebenen Abweichungen von der Gaußschen Optik wurde davon ausgegangen, dass die Ursache dafür in Abweichungen des betrachteten Laserstrahls vom idealen Gaußprofil liegt (vgl. Abschnitt 3.1.2). Selbst wenn das Strahlprofil des Lasers ein ideales Gauß-Profil darstellt, so führen die optischen Elemente, die der Strahl durchläuft, zu Abbildungsfehlern, die oft auch als Aberrationen bezeichnet werden.

Für monochromatisches Licht werden nach Seidel fünf verschiedene Arten der Aberrationen unterschieden: Sphärische Aberration, Astigmatismus, Koma, Bildfeldwölbung und Verzeichnung [8, 59].

Bei der Betrachtung der Fokussierung von Laserstrahlung überwiegt die sphärische Aberration, da Astigmatismus und Koma nur bei nicht achsparallel bzw. nicht zentral einfallendem Licht und sowohl Bildfeldwölbung als auch Verzeichnung nur bei der Abbildung ausgedehnter Objekte von Relevanz sind.

Bei der Fokussierung eines Laserstrahls tritt die sphärische Aberration zum Beispiel auf, wenn statt einer idealen (asphärischen) Linse eine sphärische Linse verwendet wird. Diese bewirkt eine stärkere Ablenkung eines einfallenden Lichtstrahls am Rand als bei einer idealen Linse. Dies hat zur Folge, dass (achsparallele) Strahlen, die die Linse an den Rändern durchlaufen auf einen Brennpunkt näher an der Linse fokussiert werden als Strahlen in der Nähe der optischen Achse. Dadurch kommt es zu einer Verbreiterung des Fokusbereichs längs der optischen Achse bzw. zu einer Aufweitung des Brennpunktes in der Brennebene.

Bei der Fokussierung eines Laserstrahls in eine mit Wasser gefüllte Küvette (mit Hilfe eines Linsensystems außerhalb der Küvette) tragen besonders die Grenzflächen zwischen Luft/Glas und Glas/Wasser zur Entstehung der sphärischen Aberration bei (vgl. Abschnitt 5.3).

$\mathrm{Zu}$ den schon in monochromatischem Licht auftretenden Aberrationen tritt bei der Betrachtung von Strahlung mit einem breiten Spektrum die chromatische Aberration hinzu. Diese kann daher bei der Betrachtung von gepulster Laserstrahlung relevant werden. 
Der unterschiedliche Brechungsindex des verwendeten Materials bei den verschiedenen Wellenlängen führt so zum Beispiel bei der achsparallelen Bestrahlung einer Einzellinse aus dispersivem Material dazu, dass Licht unterschiedlicher Wellenlänge in verschiedenen Abständen zur Linse fokussiert wird.

Bei der Betrachtung der Fokussierung ultrakurzer Pulse treten zudem raumzeitliche Effekte auf. Insbesondere kommt es durch die unterschiedlich langen Strecken, die die einzelnen Teile des Pulses abhängig von ihrer Entfernung zur optischen Achse durchlaufen, zu einer Verschiebung der Phasenfront zur Pulsfront [60, 7]. Dies kann bei kleinen Fokuswinkeln, wie sie im Rahmen dieser Arbeit betrachtet werden, allerdings vernachlässigt werden.

\subsection{Selbstfokussierung}

Wie in Abschnitt 1.3 diskutiert, wird bei hohen Intensitäten der Brechungsindex abhängig von der Intensität an der betrachteten Stelle. Der Brechnungsindex steigt dabei mit zunehmender Intensität an. Wenn sich ein Strahl mit beispielsweise einem Gauß-Profil in einem Kerr-Medium ausbreitet, dann ist der Brechungsindex für die Anteile nahe der Strahlmitte höher als für die äußeren Anteile. Demnach wird sich der Strahl in der Mitte langsamer ausbreiten als an den Rändern. Dies hat nach dem Huygensschen Prinzip (vgl. Abschnitt 3.1.5) eine Krümmung der Phasenfront nach innen zur Folge, die dazu führt, dass der Strahl sich zusammenschnürt.

Dieser Selbstfokussierung genannte Effekt ist bei optischen Systemen, bei denen hohe Intensitäten auftreten, von großer Bedeutung. So sind bei Hochleistungslasern Beschädigungen fast immer darauf zurückzuführen. Ferner wird durch die Selbstfokussierung eine Grenze bei der Transmittivität von optischen Medien gegeben (vgl. Abschnitt 3.2.1).

Die hohe praktische Relevanz führte dazu, dass der Effekt in den vergangenen Jahrzehnten theoretisch und experimentell intensiv untersucht wurde. Einen sehr guten Überblick über die Ergebnisse grundlegender Arbeiten [2, 69, 55] sowie die Gegenstände aktueller Forschung findet man in dem von R. W. Boyd, S. G. Lukishova und Y. R. Shen herausgegebenen Buch „Self-focusing: Past and Present" 9].

Im Folgenden sollen die wichtigsten Aspekte der Selbstfokussierung und insbesondere deren Einfluss auf die Fokussierung von fs-Pulsen vorgestellt werden.

\subsubsection{Townes-Soliton und kritische Leistung}

Dazu betrachten wir zunächst wieder einen (zeitunabhängigen) Laserstrahl, bevor wir in Abschnitt 3.2.5 zu gepulster Strahlung übergehen. Insbesondere 
wird eine stationäre Lösung gesucht und analysiert. Aus dieser lässt sich die kritische Leistung für die Selbstfokussierung bestimmen [29, 43, 44].

Für die Ausbreitung eines Laserstrahls in einem Kerr-Medium ergibt sich durch das Weglassen der zeitlichen Ableitungen in Gleichung (1.51) die stationäre nichtlineare Schrödinger-Gleichung:

$$
\partial_{\xi} A=\frac{i}{2 \beta_{0}} \triangle_{\perp} A+i \frac{\beta_{0} c \epsilon_{0}}{2} n_{2}|A|^{2} A .
$$

Zunächst werden neue dimensionslose Variablen

$$
r^{\star}=\frac{r}{w_{0}}, \quad \xi^{\star}=\frac{\xi}{2 \beta_{0} w_{0}^{2}} \quad \text { und } \quad A^{\star}=\beta_{0} w_{0} \sqrt{c \epsilon_{0} n_{2}} A
$$

eingeführt. Die Größe $w_{0}$ stellt zunächst nur einen beliebigen Skalierungsfaktor dar, kann aber zum Beispiel als Breite des Strahls aufgefasst werden. Mit den neuen Variablen wird Gleichung (3.16) $\mathrm{zu}$ :

$$
i \partial_{\xi^{\star}} A^{\star}=\triangle_{\perp}^{\star} A^{\star}+\left|A^{\star}\right|^{2} A^{\star} .
$$

Gesucht wird nun eine zylindersymmetrische Lösung, für die der Betrag der Einhüllenden für alle $r^{\star}$ konstant bleibt. Mit dem Separationsansatz

$$
A^{\star}=e^{i \xi^{\star}} E^{\star}\left(r^{\star}\right)
$$

erhält man [15, 16]:

$$
\frac{d^{2} E^{\star}\left(r^{\star}\right)}{d r^{\star 2}}+\frac{1}{r^{\star}} \frac{d E^{\star}\left(r^{\star}\right)}{d r^{\star}}-E^{\star}\left(r^{\star}\right)+E^{\star 3}\left(r^{\star}\right)=0 .
$$

Durch die Zylindersymmetrie und die Forderung, dass die Leistung des Strahls begrenzt sein soll, ergeben sich die folgenden Randbedingungen:

$$
\left.\frac{d E^{\star}\left(r^{\star}\right)}{d r^{\star}}\right|_{r^{\star}=0}=0 \quad \text { und } \quad \lim _{r^{\star} \rightarrow \infty} E^{\star}\left(r^{\star}\right)=0 .
$$

Die Lösung von Gleichung (3.20) mit den Randbedingungen (3.21) wird als Townes-Soliton bezeichnet [28]. In Analogie zum zeitlichen Soliton, bei der die Gruppengeschwindigkeitsdispersion durch die Nichtlinearität des Materials ausgeglichen wird, handelt es sich hierbei um das Profil, bei dem die durch die Beugung bewirkte Verbreiterung des Strahls durch die Selbstfokussierung exakt ausgeglichen wird. Allerdings ist das Townes-Soliton im Gegensatz zum zeitlichen Soliton nur metastabil.

Alternativ wird für das Townes-Soliton auch die Bezeichnung R-Profil verwendet [28]. Dies kommt daher, dass oft in Gleichung (3.20) der Buchstabe $R$ statt $E^{\star}$ verwendet wird.

Für die Gleichung (3.20) existiert keine (nichttriviale) analytische Lösung. Es kann allerdings eine numerische Lösung bestimmt werden. Dazu wird die 
Gleichung (3.20) in ein Gleichungssystem gewöhnlicher Differentialgleichungen erster Ordnung (mit der zusätzlichen Variable $U^{\star}=d E^{\star} / d r^{\star}$ ) transformiert, das mit Standardverfahren (z. B. Runge-Kutta [61]) numerisch gelöst werden kann. Die Integration wird dabei bei $r^{\star}=0$ begonnen. Als Anfangswert für $U^{\star}$ ergibt sich $U^{\star}(0)=0$ direkt aus Gleichung (3.21). $E^{\star}(0)$ kann der Literatur entnommen werden 74 oder iterativ bestimmt werden, indem $E^{\star}(0)$ so lange variiert wird, bis die zweite Randbedingung aus (3.21) hinreichend gut erfüllt ist. Es ergibt sich

$$
E^{\star}(0) \approx 2.2062
$$

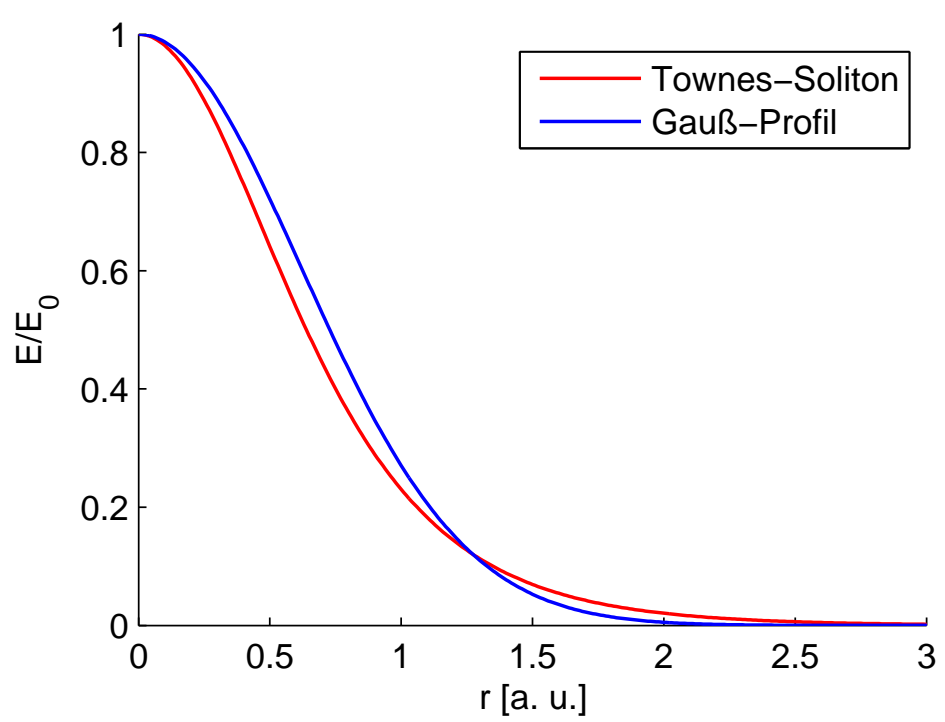

\begin{abstract}
Abbildung 3.4: Vergleich der Feldverläufe eines Townes-Solitons (rot) und eines Strahls mit Gaußschem Profil (blau). Zum Vergleich wurde auf die gleiche Intensität bei $r=0$ und identische Leistung normiert.
\end{abstract}

Abbildung 3.4 zeigt das so berechnete Townes-Soliton im Vergleich zu einem Gaußschen Strahl gleicher Maximalintensität und Leistung. Man erkennt eine große Ähnlichkeit zwischen beiden Profilen, allerdings fällt das elektrische Feld des Townes-Soliton für kleine Radien schneller ab und konvergiert dann für $r^{\star} \rightarrow \infty$ langsamer gegen 0 als das Gaußsche Strahlprofil.

Die Leistung des Townes-Solitons erhält man aus den Gleichungen (1.33) und (1.32) unter Verwendung der dimensionslosen Variablen (3.17) und der Definition von $E^{\star}(3.19)$ :

$$
\mathrm{P}_{\mathrm{cr}}^{\text {Tow }}=\frac{n_{0} c \epsilon_{0}}{2} \int_{-\infty}^{\infty} d r r|A(r)|^{2}=\frac{\lambda^{2}}{4 \pi n_{0} n_{2}} \underbrace{\underbrace{\infty}_{0} d r^{\star} r^{\star}\left|E^{\star}\left(r^{\star}\right)\right|^{2}}_{=: N_{\mathrm{cr}}^{\text {Tow }}} .
$$


Für den auftretenden Proportionalitätsfaktor $N_{\text {cr }}$ ergibt die numerische Integration einen Wert von $N_{\mathrm{cr}}^{\text {Tow }} \approx 1.8622$. Man erkennt, dass die Leistung des Townes-Solitons unabhängig von dessen Breite ist. Ein Strahl, der das Profil eines Townes-Solitons besitzt, wird sich, wenn seine Leistung $P$ kleiner als $\mathrm{P}_{\mathrm{cr}}^{\text {Tow }}$ ist, durch die Beugung verbreitern und für $\mathrm{P}>\mathrm{P}_{\mathrm{cr}}^{\text {Tow }}$ durch die dann überwiegende Selbstfokussierung immer weiter zusammenschnüren.

Für das Townes-Profil stellt $\mathrm{P}_{\mathrm{cr}}^{\text {Tow }}$ also die kritische Leistung für die Selbstfokussierung dar.

Es zeigt sich, dass die Gleichung (3.22) auch für andere Strahlprofile mit den entsprechenden Koeffizienten $N_{\text {cr }}$ gültig ist, wobei $N_{\mathrm{cr}}^{\text {Tow }}$ für diese eine untere Schranke darstellt [83]. Die Koeffizienten $N_{\text {cr }}$ müssen in der Regel numerisch bestimmt werden. Dabei ergeben sich im Allgemeinen1 nur geringfügig höhere Werte als $N_{\mathrm{cr}}^{\text {Tow }}$. So findet man für das Gauß-Profil [29]:

$$
N_{\mathrm{cr}}^{\text {Gauß }} \approx 1.8962 \text {. }
$$

Für die kritische Leistung in Wasser ergibt sich für einen Strahl mit GaußProfil und $800 \mathrm{~nm}$ Wellenlänge aus den Gleichungen (3.22) und (3.2.1) mit dem linearen und nichtlinearen Brechungsindex für Wasser (vgl. Gleichungen (1.8) und (1.19)):

$$
\mathrm{P}_{\mathrm{cr}, \mathrm{H}_{2} \mathrm{O}}^{\mathrm{Gauß}}=\frac{N_{\mathrm{cr}}^{\mathrm{Gauß}} \lambda^{2}}{4 \pi n_{0} n_{2}} \approx 3.64 \mathrm{MW}
$$

\subsubsection{Kollaps der nichtlinearen Schrödingergleichung}

Man kann zeigen, dass die Lösungen der stationären nichtlinearen Schrödingergleichung (3.16), die sich durch die Selbstfokussierung immer weiter zusammenziehen, im Fokus singulär werden [28]. Man spricht in diesem Fall von einem Strahlkollaps.

Dieser Kollaps ist natürlich unphysikalisch und kann bereits durch kleine Modifikationen der nichtlinearen Schrödingergleichung aufgefangen werden. Dazu kommt zum Beispiel das Einführen einer sättigbaren Nichtlinearität oder eines nichtlinearen Terms 5. Ordnung sowie die nichtparaxiale Erweiterung der Gleichung (3.16) in Frage ([31], Verweise in [4]).

Bei gepulster Strahlung verhindert zudem eine (ausreichend große) normale Gruppengeschwindigkeitsdispersion das Auftreten von Singularitäten (vgl. Abschnitt 3.4).

Der wichtigste Effekt, der den Kollaps des Strahls verhindert, ist allerdings das Entstehen eines Plasmas, das in Kapitel 4 detailliert beschrieben wird. Dieses hat einen defokussierenden Effekt (vgl. Abschnitt 5.1) und spielt eine wichtige Rolle bei der Bildung von Filamenten (vgl. Abschnitt 3.2.6).

\footnotetext{
${ }^{1}$ Ausnahmen bilden zum Beispiel Ringprofile [55]
} 


\subsubsection{Kollapsprofil}

In Abschnitt 3.2.1 wurde dargestellt, dass das Townes-Soliton das Profil mit der kleinsten kritischen Leistung für die Selbstfokussierung darstellt. Bei der Betrachtung des Strahlkollaps zeigt sich noch eine weitere wichtige Eigenschaft dieses Profils: Mit wenigen Ausnahmen ist das Strahlprofil $E^{\text {Kollaps }}$ in der Nähe des Brennpunktes $z_{\mathrm{sf}}$ unabhängig vom ursprünglichen Profil das eines (modulierten) Townes-Profils $E^{\star}[28]$ :

$$
\left|E^{\text {Kollaps }}(r, z)\right| \sim \frac{1}{l(z)} E^{\star}\left(\frac{r}{l(z)}\right), \quad \lim _{z \rightarrow z_{\mathrm{sf}}} l(z)=0 .
$$

Für Strahlung, dessen Leistung nicht viel größer als die kritische Leistung $\mathrm{P}_{\mathrm{cr}}^{\text {Tow }}$ ist, gilt diese Aussage für beliebige Strahlprofile und kann mathematisch bewiesen werden [56]. In diesem Fall kann die Veränderung der Breite $l(z)$ (engl.: blowup rate) wie folgt angegeben werden:

$$
l(z) \sim\left(\frac{\frac{z_{\mathrm{sf}}-z}{2 \beta_{0} w_{0}^{2}}}{\ln \left|\ln \left(\frac{z_{\mathrm{sf}}-z}{2 \beta_{0} w_{0}^{2}}\right)\right|}\right)^{1 / 2} .
$$

Diese Abhängigkeit wird erst sehr kurz vor dem Kollaps erreicht, numerische Simulationen [31] zeigen, dass $l(z)$ in größeren Abständen zum Brennpunkt durch die Wurzel $\sqrt{\left(z_{\mathrm{sf}}-z\right) / 2 \beta_{0} w_{0}^{2}}$ genähert werden kann.

Wie man in Gleichung (3.22) erkennen kann, ist die Leistung des TownesSolitons unabhängig von dessen Breite. Daher erhält man für die Leistung des kollabierenden Strahls mit Gleichung (3.24) immer die kritische Leistung $\mathrm{P}_{\mathrm{cr}}^{\text {Tow }}$. Für andere Strahlprofile als das Townes-Soliton kollabiert nur ein Teil des Strahles, dies findet man in der Literatur unter der Bezeichnung partial beam collapse [28]. Es ist allerdings zu beachten, dass diese Überlegung für den Strahlkollaps der (unmodifizierten) nichtlinearen Schrödingergleichung gilt. In realen Systemen wird - wie bereits in Abschnitt 3.2.2 angesprochen insbesondere durch die Entstehung eines Plasmas der Strahlkollaps verhindert. Dessen Entstehung hängt insbesondere auch von der Leistung ab, die nicht in das Townes-Profil eingeht. Zudem kann es sein, dass das Townes-Profil auch erst sehr kurz vor dem theoretischen Kollaps erreicht wird - zu einem Zeitpunkt, an dem die Ausbildung eines Plasmas schon relevant wird.

Das Ausbilden des Townes-Profils im Kollaps wurde auch experimentell bestätigt und insbesondere in Glas für elliptische Profile sowie verzerrte Gauß-Profile [57] als auch in Wasser für Gaußsche Profile [35] nachgewiesen.

Strahlen mit Ringprofilen [82] und sog. Super-Gauß-Profilen [35], deren Leistung zudem ein Vielfaches der kritischen Leistung überschreitet, stellen eine Ausnahme dar. In beiden Fällen bildet sich im Kollaps ein Ringprofil [30] aus, das allerdings stark zum Aufbrechen in Filamente neigt (vgl. Abschnitt 3.2.6). 


\subsubsection{Brennweite der Selbstfokussierung}

Die Stelle, auf die ein Strahl mit $\mathrm{P}>\mathrm{P}_{\mathrm{cr}}$ fokussiert wird, kann analytisch nur näherungsweise bestimmt werden [43, 44]. Für eine exakte Bestimmung der Brennweite muss die stationäre nichtlineare Schrödingergleichung numerisch gelöst werden (vgl. Abschnitt 3.3.3). Berechnungen von Marburger et al. 20, 55 haben ergeben, dass für einen Strahl mit einem Gauß-Profil und einem Strahlradius $w$ die Brennweite der Selbstfokussierung $z_{\mathrm{sf}}$ durch folgende Näherungsformel beschrieben werden kann:

$$
z_{\mathrm{sf}}=\frac{0.1835 \beta_{0} w^{2}}{\left\{\left[\left(\mathrm{P} / \mathrm{P}_{\mathrm{cr}}\right)^{1 / 2}-0.852\right]^{2}-0.0219\right\}^{1 / 2}} .
$$

Es kann gezeigt werden, dass die stationäre nichtlineare Schrödingergleichung (3.16) invariant unter der sogenannten Linsentransformation (engl. lens transformation) [77] ist. Dies hat zur Folge, dass eine Linse in einem nichtlinearen Medium den gleichen Effekt hat wie in einem linearen Medium [28]. Um die Brennweite für einen bereits fokussierten (oder defokussierten) Strahl in einem nichtlinearen Medium zu bestimmen, kann daher die Linsengleichung verwendet werden. Für einen Strahl, für den sich nach Gleichung (3.26) eine Brennweite für die Selbstfokussierung von $z_{\text {sf }}$ ergibt, erhält man bei vorheriger Fokussierung durch eine Linse der Brennweite $z_{\mathrm{f}}$ eine kombinierte Brennweite von $z_{\mathrm{f}+\mathrm{sf}}$ :

$$
\frac{1}{z_{\mathrm{f}+\mathrm{sf}}}=\frac{1}{z_{\mathrm{f}}}+\frac{1}{z_{\mathrm{sf}}}
$$

\subsubsection{Selbstfokussierung von Pulsen}

Bisher wurde der Effekt der Selbstfokussierung für kontinuierliche Strahlung betrachtet. In der Regel werden die für die Selbstfokussierung benötigten Leistungen jedoch nur bei gepulster Strahlung erreicht.

Für die Betrachtung der Selbstfokussierung bei ultrakurzen Pulsen wird der Laserpuls zeitlich in Intervalle, die oft als Zeitscheiben (engl.: time slices) bezeichnet werden, unterteilt. Kann der Einfluss der Gruppengeschwindigkeitsdispersion vernachlässigt werden, so kann die Entwicklung einer Zeitscheibe unabhängig von den anderen betrachtet werden [18. Wie in Abschnitt 1.18 diskutiert, wird ferner die Wirkung der Nichtlinearität als instantan betrachtet, man spricht in diesem Fall von einem quasi-stationären Modell [70]. Jeder Zeitscheibe kann eine (mittlere) Leistung $\mathrm{P}$ zugeordnet werden. Liegt diese über der kritischen Leistung der Selbstfokussierung $P_{c r}$, so kollabiert der zugehörige Anteil des Laserpulses. Der Brennpunkt $z_{\mathrm{sf}}$ ist dabei von der Leistung der Zeitscheibe abhängig und kann mit Hilfe von Gleichung (3.26) bestimmt werden. Durch die verschiedenen Brennweiten der Zeitscheiben wandert der 
Brennpunkt entlang der optischen Achse, dies wird in der englischsprachigen Literatur als moving focus bezeichnet [70].

\subsubsection{Filamente}

Wie in Abschnitt 3.2.2 diskutiert, wird im Experiment der bei Lösungen der nichtlinearen Schrödingergleichung auftretende Strahlkollaps durch verschiedene Effekte verhindert. Es zeigt sich, dass die bei hohen Intensitäten auftretende Ionisierung des Materials den größten Einfluss hat. Das entstehende Plasma verhindert durch Absorption und Defokussierung (vgl. Kapitel 5), dass die Intensität immer weiter ansteigt.

Damit kann der sich bewegende Fokus (vgl. Abschnitt 3.2.5) in zeitlicher Mittelung als Filament betrachtet werden [17, 12]. In Luft wurden für fs-Pulse Filamente von mehrern Metern Länge beobachtet [11].

Innerhalb solcher Filamente kann es durch die auftretenden nichtlinearen Effekte zu einer starken Verbreiterung des Pulspektrums kommen. Dies kann für die Weißlichterzeugung und bei der Pulskompression ausgenutzt werden.

Bei einem nicht idealen Strahlprofil kann es zudem (durch Amplituden- oder Phasenstörungen) vorkommen, dass durch die Selbstfokussierung mehrere Foki entstehen, die die Ausbildung multipler Filamente bewirken. So kann bei der optischen Kavitation mit fs-Laserpulsen bei hohen Energien die Ausbildung von Filamenten vor der Entstehung des eigentlichen Plasmas beobachtet werden 33 .

\subsection{Angewendetes Verfahren zur Berechnung fo- kussierter Pulse}

Die Simulation fokussierter Laserpulse erfolgt in zwei Schritten: Zunächst wird die Form des Laserpulses kurz vor dem Fokus bestimmt, die dann im zweiten Schritt für die Berechnung der Pulsausbreitung beim Durchlaufen des Fokusbereichs verwendet wird.

\subsubsection{Pulsform vor dem Fokusbereich}

Zunächst wird davon ausgegangen, dass in hinreichend großer Entfernung vom Fokus die nichtlinearen Effekte noch keine Rolle spielen. Berücksichtigt man zusätzlich keine raumzeitlichen Effekte (vgl. Abschnitt 3.1.6) kann die räumliche Ausdehnung des Pulses unabhängig von dessem zeitlichen Verlauf betrachtet werden. Im einfachsten Fall von Pulsen mit einem Gaußschen Strahlprofil 
geschieht dies analytisch mit Hilfe der Gleichungen (3.3). Liegt ein anderes Strahlprofil vor oder sollen Einflüsse des im Experiment verwendeten Linsensystems sowie der Küvette berücksichtigt werden (vgl. Abschnitt 3.1.6), wird das Strahlprofil kurz vor dem Fokus durch Auswertung des Kirchhoffschen Beugungsintegrals in Fresnelscher Näherung nach Gleichung (3.15) bestimmt. Durch die Anwendung von Besselfunktionen bei rotationssymmetrischen Strahlprofilen kann die räumliche Ausdehnung des elektrischen Feldes auch an einer großen Anzahl von Punkten schnell bestimmt werden. Alternative Verfahren zur Lösung der paraxialen Wellengleichung bzw. des Beugungsintegrals mit Hilfe von Fouriertransformierten sind bei der Untersuchung fokussierter Pulse weniger gut geeignet, da durch die starke Verringerung der Strahlbreite eine extrem feine Diskretisierung oder ein mehrschrittiges Verfahren nötig wird.

\subsubsection{Pulsausbreitung im Fokusbereich}

Mit dem zweiten Schritt der Simulation wird einige (ungefähr zehn) Rayleighlängen vor dem geometrischen Fokus begonnen. Dabei wurde sich am Vorgehen von Arnold [3] orientiert und der Startpunkt durch Vergleichsrechnungen verifiziert.

Die Berechnung der Pulsausbreitung im Bereich um den Fokus wird wie für den eindimensionalen Fall in Abschnitt 2.1 beschrieben durchgeführt. Die auftretenden räumlichen Ableitungen werden mit Hilfe des Crank-Nicolson-Verfahrens (vgl. Abschnitt 3.3.3) berechnet. Die Diskretisierung senkrecht zur Ausbreitungsrichtung muss dabei insbesondere so bemessen werden, dass die Auflösung für einen Puls im Fokus noch ausreichend ist.

\subsubsection{Crank-Nicolson-Verfahren}

Betrachtet man in Gleichung (1.51) nur den Einfluss des für die Beugung verantwortlichen Terms und vernachlässigt (vorübergehend) die auftretende zeitliche Ableitung, ergibt sich mit einer geeigneten Konstanten $k_{\perp}$ eine Gleichung vom Typ einer Wärmeleitungsgleichung:

$$
\partial_{\xi} A=k_{\perp} \triangle_{\perp} A
$$

Diese wird mit dem Crank-Nicolson-Verfahren gelöst [19]. Bei diesem handelt es sich um ein Verfahren auf Basis von finiten Differenzen. Es ergibt sich aus der Kombination von explizitem und implizitem Eulerverfahren. Betrachtet man nur die Ableitungen in einer Raumrichtung $\left(\triangle_{\perp}=\partial_{x}^{2}\right)$, so ergibt sich für einen Schritt nach dem Crank-Nicolson-Verfahren:

$$
\frac{A_{i}^{n+1}-A_{i}^{n}}{\Delta \xi}=k_{\perp} \frac{1}{2}\left(\frac{A_{i+1}^{n}-2 A_{i}^{n}+A_{i-1}^{n}}{\Delta x^{2}}+\frac{A_{i+1}^{n+1}-2 A_{i}^{n+1}+A_{i-1}^{n+1}}{\Delta x^{2}}\right) .
$$


Dabei wurde $A(x, \xi)$ auf einem regulären Gitter diskretisiert. $n=1, \ldots, n_{\max }$ und $i=1, \ldots, i_{\max }$ bezeichnen die zugehörigen Indizes für die $\xi$ - und die $x$ Achse, der Abstand zwischen zwei benachbarten Diskretisierungspunkten ist durch $\Delta \xi$ und $\Delta x$ gegeben. Der Term auf der linken Seite von Gleichung (3.28) ergibt dann eine lineare Approximation der zeitlichen Ableitung am Ort $x_{i}$. Die Terme auf der rechten Seite stellen die zweite Ableitung nach $x$ um den Ort $x_{i}$ und den Punkten $\xi_{n}$ und $\xi_{n+1}$ dar.

Das Verfahren lässt sich auf mehr Dimensionen erweitern, indem ein entsprechend höherdimensionales Gitter verwendet wird. Alternativ kann man - bei Vorliegen einer Zylindersymmetrie - den Einfluss der zusätzlichen Dimension durch Verwendung des Laplace-Operators in Zylinderkoordinaten berücksichtigen. Dieser kann wie folgt als Differenz dargestellt werden:

$$
\triangle_{\perp} A=\left(\frac{\partial^{2}}{\partial r^{2}}+\frac{1}{r} \frac{\partial}{\partial r}\right) A \rightarrow \frac{A_{i+1}^{n}-2 A_{i}^{n}+A_{i-1}^{n}}{\Delta r^{2}}+\frac{1}{r_{i}} \frac{A_{i+1}^{n}-A_{i-1}^{n}}{2 \Delta r} .
$$

Damit wird Gleichung (3.28) $\mathrm{zu}$

$$
\begin{aligned}
A_{i}^{n+1}-A_{i}^{n}=\underbrace{k_{\perp} \frac{\Delta \xi}{2}}_{=: s}\left(\frac{A_{i+1}^{n}-2 A_{i}^{n}+A_{i-1}^{n}}{\Delta r^{2}}+\frac{1}{r_{i}} \frac{A_{i+1}^{n}-A_{i-1}^{n}}{2 \Delta r}\right. \\
\left.\quad+\frac{A_{i+1}^{n+1}-2 A_{i}^{n+1}+A_{i-1}^{n+1}}{\Delta r^{2}}+\frac{1}{r_{i}} \frac{A_{i+1}^{n+1}-A_{i-1}^{n+1}}{2 \Delta r}\right) .
\end{aligned}
$$

Fasst man die Terme geeignet zusammen, so erhält man:

$$
\begin{aligned}
& \underbrace{-s\left(\frac{1}{\Delta r}+\frac{1}{2 r_{i} \Delta r^{2}}\right)}_{\lambda_{i+1}^{n+1}} A_{i+1}^{n+1}+\underbrace{\left(1+\frac{2 s}{\Delta r^{2}}\right)}_{\lambda_{i}^{n+1}} A_{i}^{n+1} \underbrace{-s\left(\frac{1}{\Delta r}-\frac{1}{2 r_{i} \Delta r^{2}}\right)}_{\lambda_{i-1}^{n+1}} A_{i-1}^{n+1} \\
& =\underbrace{s\left(\frac{1}{\Delta r}+\frac{1}{2 r_{i} \Delta r^{2}}\right)}_{\lambda_{i+1}^{n}} A_{i+1}^{n}+\underbrace{\left(1-\frac{2 s}{\Delta r^{2}}\right)}_{\lambda_{i}^{n}} A_{i}^{n}+\underbrace{s\left(\frac{1}{\Delta r}-\frac{1}{2 r_{i} \Delta r^{2}}\right)}_{\lambda_{i-1}^{n}} A_{i-1}^{n} .
\end{aligned}
$$

Stellt man für jeden der Diskretisierungspunkte eine entsprechende Gleichung auf, so ergibt sich ein Gleichungssystem, das in Matrixschreibweise die folgende Form besitzt:

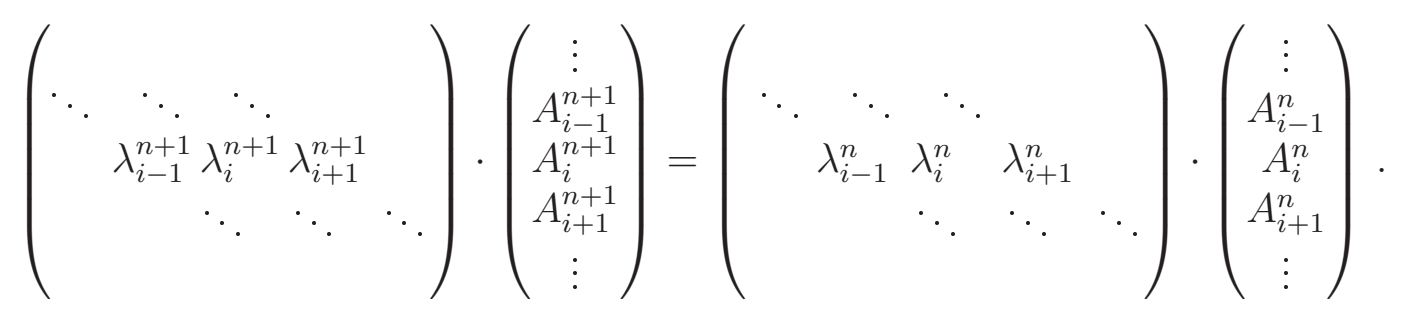

Um dieses Gleichungssystem nach den unbekannten $A_{1}^{n+1}, \ldots, A_{i_{\max }+1}$ lösen zu können, muss die Matrix mit den Koeffizienten $\lambda_{1}^{n+1}, \ldots, \lambda_{i_{\max }}^{n+1}$ invertiert werden. Da es sich bei dieser um eine tridiagonale Matrix handelt, kann das 
Gleichungssystem mit einem speziell darauf abgestimmten Algorithmus 61] effizient gelöst werden.

\subsection{Beispiel: Pulsaufspaltung}

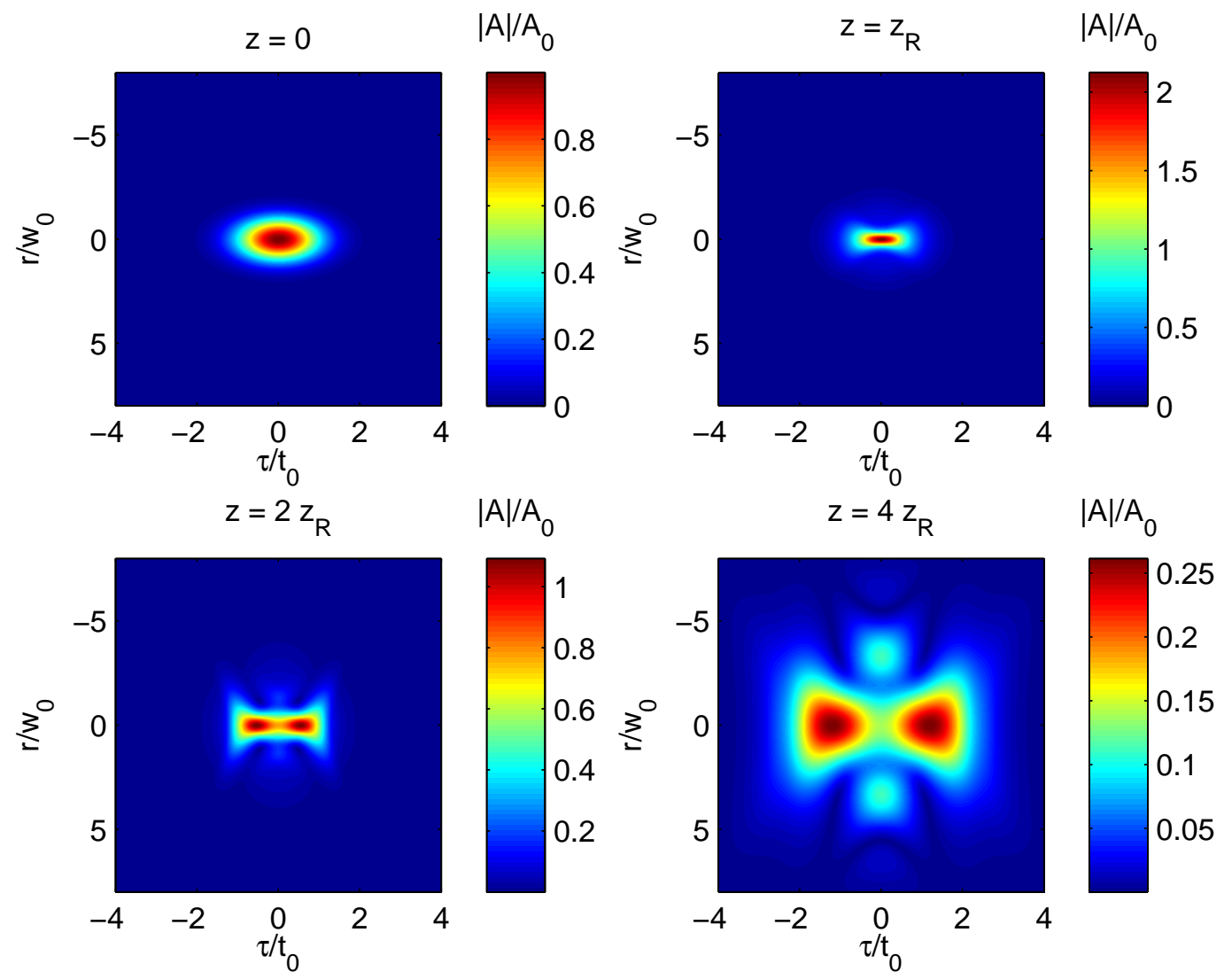

Abbildung 3.5: Aufspalten eines Pulses beim Durchlaufen des Fokus bei normaler GVD (Parameter nach Chernev [14])

Wie in Abschnitt 3.2 .2 beschrieben, kann durch eine normale Gruppengeschwindigkeitsdispersion der Strahlkollaps verhindert werden. Unter bestimmten Bedingungen besteht die Möglichkeit, dass sich der Puls zeitlich in zwei Teile aufspaltet. Dieses Phänomen wird in der englischsprachigen Literatur als pulse splitting bezeichnet [65].

Zur Verifikation der Simulation wurde die Propagation eines solchen Pulses berechnet, dessen Parameter von Chernev et al. [14] übernommen wurden. $\mathrm{Zu}$ Beginn besitzt der Puls sowohl zeitlich als auch räumlich ein Gaußsches Profil (vgl. Gleichungen (1.29) und (3.2)). Die Leistung wird mit $P=1.437 \mathrm{P}_{\text {cr }}$ angesetzt. Damit erhält man mit den Gleichungen (3.26) und (3.4) für die 
Brennweite der Selbstfokussierung:

$$
z_{\mathrm{sf}}=1.170 z_{\mathrm{R}} .
$$

Ferner wird der für die Gruppengeschwindigkeitsdispersion entscheidende Koeffizient $\beta_{2}$ (vgl. Gleichung (1.39) ) wie folgt gewählt:

$$
\beta_{2}=0.0755 \frac{z_{\mathrm{R}}}{t_{0}^{2}}
$$

Abbildung 3.5 zeigt die so berechneten Pulsformen. Man erkennt, wie sich der Puls zunächst räumlich durch die Selbstfokussierung zusammenschnürt. Bei $z=z_{\mathrm{R}}$ wäre der Puls ohne GVD nach Gleichung (3.29) kurz vor dem Kollabieren. Die Gruppengeschwindigkeitsdispersion verhindert den Kollaps und ein in zwei zeitliche Anteile aufgespaltener Puls entsteht, der sich im weiteren Verlauf räumlich wieder ausdehnt.

Der Vergleich der berechneten Pulsform für $z=4 z_{\mathrm{R}}$ mit Abbildung 2 aus [14] zeigt eine sehr gute Übereinstimmung.

Ferner wurden zum Test der Simulation verschiedene andere Berechnungen durchgeführt. So kann bei der linearen Ausbreitung Gaußscher Pulse das numerisch bestimmte Ergebnis mit der analytischen Lösung (vgl. Gleichung (3.3), s. a. [71) verglichen werden. Zum Test der Implementierung der nichtlinearen Terme wurde die Ausbreitung von Pulsen mit Townes-Profil berechnet (vgl. Abschnitt 3.2.1). 


\section{Kapitel 4}

\section{Prozesse der Plasmabildung und -dynamik}

Nach den Betrachtungen zur Ausbreitung von Laserpulsen in den vorangegangenen Kapiteln werden im Folgenden die Entstehung und die Eigenschaften des durch die Pulse im Fokus erzeugten Plasmas diskutiert. Dazu wird zunächst ein Überblick über die bei der Plasmabildung und -dynamik auftretenden Phänomene gegeben.

\section{1 Überblick}

Wie in Abschnitt 3.2.2 diskutiert, wird die Lösung der nichtlinearen Schrödingergleichung bei einem Puls, der sich durch die Selbstfokussierung immer weiter zusammenzieht, an einem bestimmten Punkt singulär. In realen Systemen wird dies durch das Entstehen eines Plasmas verhindert. Dieses kann insbesondere zu einer Erhitzung des Materials führen, die bei Flüssigkeiten die Entstehung einer Kavitationsblase bewirken kann. In diesem Fall spricht man von einem optischen Durchbruch (vgl. Abschnitt 4.6) [70].

Ein weit verbreiteter Ansatz zur Modellierung des Verhaltens der Elektronen im optischen Durchbruch geht auf Sacchi [66] zurück, der gezeigt hat, dass die Behandlung von Wasser als amorphen Halbleiter mit einer Bandlücke von $\mathrm{E}_{\mathrm{b}}=6.5 \mathrm{eV}$ sehr gute Übereinstimmung mit experimentellen Beobachtungen ergibt. Ein gebundenes Elektron entspricht in diesem Modell einem Elektron im Valenzband des Halbleiters, ein freies Elektron einem im Leitungsband. Der Prozess der Ionisation wird folglich durch den Übergang eines Elektrons vom Valenz- ins Leitungsband beschrieben.

Für die in dieser Arbeit betrachteten Laserwellenlängen ist Wasser bei geringen Intensitäten ein transparentes Medium. Es findet keine Absorption statt, da die Energie der Photonen (zum Beispiel für $\lambda=800 \mathrm{~nm} \mathrm{E}=1.55 \mathrm{eV}$ ) nicht 
ausreicht, um Elektronen vom Valenzband in das Leitungsband überführen zu können. Bei ausreichend hoher Intensität des Laserlichtes existieren allerdings zwei Mechanismen der Photoionisation, durch die dies möglich wird.

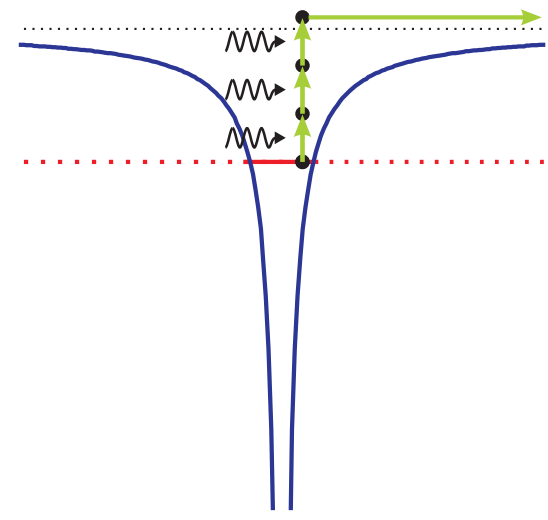

(a) Multiphotonenionisation

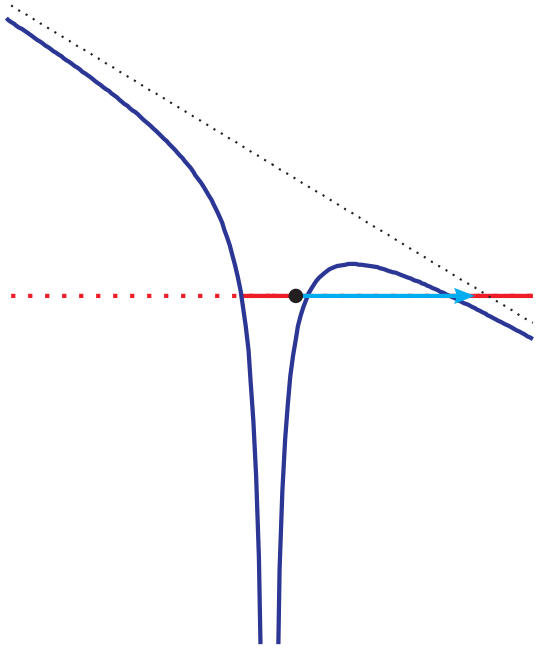

(b) Tunnelionisation

Abbildung 4.1: Schematische Darstellung der Multiphotonenabsorption und Tunnelionisation für ein einzelnes Atom nach [36].

So steigt bei hoher Intensität die Wahrscheinlichkeit, dass genügend Photonen vorhanden sind, die gleichzeitig von einem Atom des Mediums absorbiert werden können. Ist die Gesamtenergie der absorbierten Photonen größer als die Bindungsenergie eines Elektrons, so geht dieses in einen ungebundenen Zustand über (vgl. Abb. 4.1 (a)). Diesen Prozess nennt man Multiphotonenionisation.

Eine weitere Möglichkeit der Ionisation eines Elektrons trotz zu geringer Energie des eingestrahlten Lichts stellt die Tunnelionisation dar. Diese tritt auf, wenn die Intensität des eingestrahlten Lichts so groß ist, dass das äußere elektrische Feld die Potentialbarriere so stark verzerrt, dass die gebundenen Elektronen diese mit einer gewissen Wahrscheinlichkeit überwinden können. Diese Situation wird in Abbildung 4.1 (b) schematisch dargestellt.

Keldysh hat bereits 1965 ein gemeinsames Modell für die Multiphotonen- und Tunnelionsation aufgestellt [42]. Dieses findet bis heute breite Anwendung und wird auch in dieser Arbeit verwendet. Es wird im nächsten Abschnitt vorgestellt.

Neben den Mechanismen der Photoionisation spielt der Prozess der inversen Bremsstrahlungsabsorption eine wichtige Rolle bei der Plasmaentstehung durch ultrakurze Laserpulse. Durch diesen kann bei dem Stoß eines freien Elektrons mit einem Wassermolekül ein Photon absorbiert werden. Dieses Elektron 
kann dadurch genug Energie aufnehmen, um ein anderes Elektron aus der Hülle eines Moleküls herauszuschlagen (Stoßionisation). Die freien Elektronen können erneut Energie durch Stöße mit Wassermolekülen aufnehmen und weitere freie Elektronen erzeugen. Zwei Stöße können nicht beliebig schnell hintereinander erfolgen, so dass die neuen freien Elektronen erst nach einer bestimmten Zeit erzeugt werden, man spricht deshalb von einer Kaskadenionisation. Unter der Voraussetzung einer ausreichend hohen Intensität verdoppelt sich die Anzahl der freien Elektronen bei jeder Kaskade. Daher ist auch die Bezeichnung Lawinenionisation gebräuchlich.

Für die Beschreibung der Kaskadenionisation wird das Drude-Modell verwendet, das in Abschnitt 4.3.1 vorgestellt wird.

Für den Start der Kaskadenionisation wird zunächst mindestens ein freies Elektron benötigt, das in der Regel durch Photoionisation erzeugt wird. In Abbildung 4.2 wird das Zusammenspiel der verschiedenen Prozesse schematisch dargestellt.

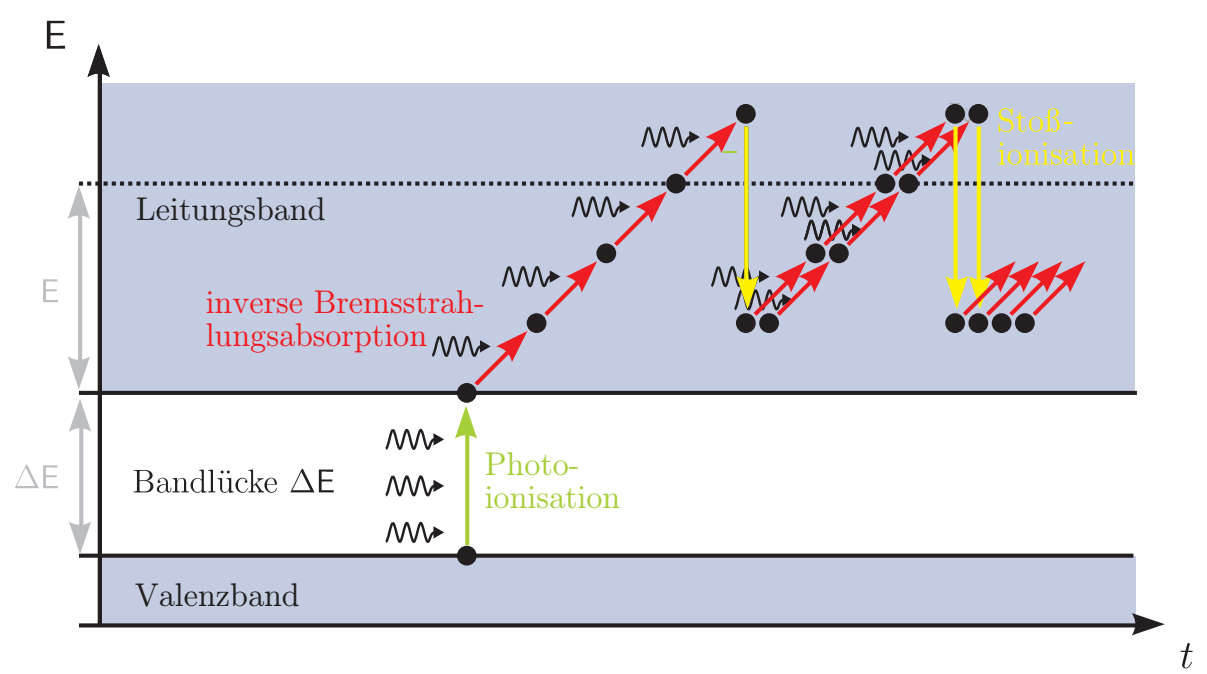

Abbildung 4.2: Das Zusammenspiel zwischen Photoionisation und Kaskadenionisation (Grafik nach Vogel [80])

Neben den Prozessen zur Entstehung freier Elektronen existieren auch Effekte, die zu einer Reduzierung der Elektronendichte im Fokus führen: Dies sind insbesondere die Diffusion von Elektronen aus dem Fokusvolumen sowie die Rekombination.

Im Folgenden werden die vorgestellten Prozesse detailliert beschrieben und zum einen ein Modell zur Beschreibung des entstehenden Plasmas vorgestellt und zum anderen dessen Auswirkung auf die Ausbreitung der Laserstrahlung betrachtet. 


\subsection{Keldysh-Theorie der Photoionisation}

Zur Bestimmung der Wahrscheinlichkeit für die Photoionisation wird auf Berechnungen von Keldysh 42 zurückgegriffen, der Ionisationswahrscheinlichkeiten sowohl für einzelne Atome ( $\rightarrow$ Gase) als auch für Festkörper berechnet hat. Die Ergebnisse für Festkörper können auf Wasser übertragen werden, wenn dieses dem Modell von Sacchi [66] entsprechend (vgl. Abschnitt 4.1) als Halbleiter mit im Vergleich zur Energie der eingestrahlten Photonen großer Bandlücke betrachtet wird.

\subsubsection{Wahrscheinlichkeit der Photoionisation}

Für die Ionisationsrate bei Einstrahlung eines elektrischen Feldes $E_{0} \cos \left(\omega_{0} t\right)$ der Frequenz $\omega_{0}$ und der (skalar und reell angenommenen) Amplitude $E_{0}$ in einem Material der Bandlücke $\mathrm{E}_{\mathrm{b}}$ gilt [42]:

$$
\eta_{\mathrm{ph}}=\frac{2 \omega_{0}}{9 \pi}\left(\frac{m^{\prime} \omega_{0}}{\gamma \Gamma \hbar}\right)^{\frac{3}{2}} Q\left(\gamma, \frac{\mathrm{E}_{\mathrm{eff}}}{\hbar \omega_{0}}\right) \exp \left(-\pi\left\langle\frac{\mathrm{E}_{\mathrm{eff}}}{\hbar \omega_{0}}+1\right\rangle \cdot \frac{\mathrm{K}(\gamma \Gamma)-\mathrm{E}(\gamma \Gamma)}{\mathrm{E}(\Gamma)}\right)
$$

mit

$$
\begin{aligned}
& Q(\gamma, x)=\sqrt{\frac{\pi}{2 \mathrm{~K}(\Gamma)}} \cdot \sum_{n=0}^{\infty}[ \exp \left(-\pi n \frac{\mathrm{K}(\gamma \Gamma)-\mathrm{E}(\gamma \Gamma)}{\mathrm{E}(\Gamma)}\right) \\
&\left.\cdot \Phi\left(\sqrt{(2\langle x+1\rangle-2 x+n) \frac{\pi^{2}}{2 \mathrm{~K}(\Gamma) \mathrm{E}(\Gamma)}}\right)\right]
\end{aligned}
$$

Dabei bezeichnet $E_{\text {eff }}$ die effektive Bandlücke. In dieser ist zusätzlich zu $E_{b}$ die Schwingungsenergie der Elektronen im Leitungsband berücksichtigt, die bei der Ionisation ebenfalls aufgebracht werden muss:

$$
\mathrm{E}_{\mathrm{eff}}=\frac{2 \mathrm{E}(\Gamma)}{\pi \gamma \Gamma} \mathrm{E}_{\mathrm{b}} .
$$

Eine wichtige Größe stellt der Keldysh-Parameter $\gamma$ dar, der mit der Definition der Intensität in Gleichung (1.32) durch

$$
\gamma=\frac{\omega_{0} \sqrt{m^{\prime} \mathrm{E}_{\mathrm{b}}}}{e E_{0}}=\frac{\omega_{0}}{e} \sqrt{\frac{m^{\prime} \mathrm{E}_{\mathrm{b}} c \epsilon_{0} n}{2 I}}
$$

gegeben ist. Ferner ist $\Gamma$ gegeben durch

$$
\Gamma=1 / \sqrt{1+\gamma^{2}} .
$$

$m^{\prime}$ bezeichnet die reduzierte Masse, für die gilt [42]:

$$
\frac{1}{m^{\prime}}=\frac{1}{m_{e}}+\frac{1}{m_{h}}
$$


Für Wasser können - Vogel et al. 80] folgend - die auftretende Masse für ein Elektron $m_{e}$ und ein Loch $m_{h}$ als gleich groß angenommen werden. Damit ergibt sich für die reduzierte Masse $m^{\prime}$ :

$$
m^{\prime}=\frac{m_{e}}{2} .
$$

$\mathrm{K}(\cdot)$ und $\mathrm{E}(\cdot)$ bezeichnen die vollständigen elliptischen Integrale der ersten und zweiten Art [13] und $\Phi(x)$ das sogenannte Dawson-Integral [21], für das gilt:

$$
\Phi(x)=\exp \left(-x^{2}\right) \int_{0}^{x} \exp \left(y^{2}\right) d y .
$$

$\langle x\rangle$ gibt die größte ganze Zahl an, die kleiner oder gleich $x$ ist. Insbesondere bezeichnet $\mathrm{k}$ die Anzahl der nötigen Photonen, um die für die Ionisierung nötige Energie $\mathrm{E}_{\mathrm{eff}}$ (vgl. Gleichung (4.3) ) aufzubringen:

$$
\mathrm{k}=\left\langle\frac{\mathrm{E}_{\mathrm{eff}}}{\hbar \omega_{0}}+1\right\rangle .
$$

Bei der Betrachtung der Ionisationsrate können zwei Näherungen betrachtet werden. Inwieweit diese angewendet werden können, kann anhand des in Gleichung (4.4) eingeführten Keldysh-Parameters $\gamma$ entschieden werden. Für $\gamma \gg 1$ ist für die Photoionisation der Effekt der Multiphotonenionisation entscheidend, für $\gamma \ll 1$ überwiegt die Tunnelionisation.

\subsubsection{Muliphotonenabsorption}

Für $\gamma \gg 1$ (Regime der Multiphotonenionisation) gibt Keldysh [42] an:

$$
\begin{aligned}
\eta_{\mathrm{ph}} \approx \frac{2 \omega_{0}}{9 \pi}\left(\frac{m^{\prime} \omega_{0}}{\hbar}\right)^{\frac{3}{2}} & \cdot \Phi\left(\sqrt{2 \mathrm{k}-\frac{2 \mathrm{E}_{\mathrm{eff}}}{\hbar \omega_{0}}}\right) \\
\cdot & \exp \left\{2 \mathrm{k} \cdot\left(1-\frac{e^{2} E_{0}^{2}}{4 m^{\prime} \omega_{0}^{2} \mathrm{E}_{\mathrm{eff}}}\right)\right\}\left(\frac{e^{2} E_{0}^{2}}{16 m^{\prime} \omega_{0}^{2} \mathrm{E}_{\mathrm{eff}}}\right)^{\mathrm{k}}
\end{aligned}
$$

Dann wird Gleichung (4.3) zu:

$$
\mathrm{E}_{\mathrm{eff}} \approx \mathrm{E}_{\mathrm{b}}+\frac{e^{2} E_{0}^{2}}{4 m^{\prime} \omega_{0}^{2}}
$$

Für den Bereich sehr kleiner Intensitäten wird $e^{2} E_{0}^{2} / 4 m^{\prime} \omega_{0}^{2} \mathrm{E}_{\text {eff }}$ in Gleichung (4.8) vernachlässigbar gegen 1 und die Ionisationswahrscheinlichkeit proportional zur k-ten Potenz der Intensität (vgl. Kennedy [45]). 


\subsubsection{Tunnelionisation}

Für $\gamma \ll 1$ überwiegt der Prozess der Tunnelionisation. Für diesen Fall wird Gleichung (4.1) $\mathrm{zu}$ 42]:

$$
\begin{aligned}
\eta_{\mathrm{ph}} \approx \frac{2 \mathrm{E}_{\mathrm{b}}}{9 \pi^{2} \hbar}\left(\frac{m^{\prime} \mathrm{E}_{\mathrm{b}}}{\hbar^{2}}\right)^{\frac{3}{2}}\left(\frac{e \hbar E_{0}}{m^{\prime 1 / 2} \mathrm{E}_{\mathrm{b}}^{3 / 2}}\right)^{\frac{5}{2}} & \quad \cdot \exp \left(-\frac{\pi}{2} \frac{m^{\prime 1 / 2} \mathrm{E}_{\mathrm{b}}^{3 / 2}}{e \hbar E_{0}}\left(1-\frac{1}{8} \frac{\omega_{0}^{2} m^{\prime} \mathrm{E}_{\mathrm{b}}}{e^{2} E_{0}^{2}}\right)\right) .
\end{aligned}
$$

Man erkennt, dass für hohe Intensitäten $\left(\frac{1}{8} \gamma^{2} \ll 1\right)$ die Ionisationsrate unabhängig von der Frequenz des eingestrahlten Lichtes wird. Kann der exponentielle Anteil vernachlässigt werden, so gilt:

$$
\eta_{\mathrm{ph}} \sim E_{0}^{5 / 2} \text {. }
$$

\subsubsection{Berechnung der Ionisationsrate}

Die bei der Berechnung der Ionisationswahrscheinlichkeit für die Photoionisation (vgl. Gleichung (4.1)) auftretenden elliptischen Integrale können mit Hilfe einer Näherungsformel [61] bestimmt und das Dawson-Integral aus Gleichung (4.6) über die folgende Summe [26] approximiert werden:

$$
\Phi(x)=\exp \left(-x^{2}\right) \sum_{r=0}^{\infty} \frac{x^{2 r+1}}{r !(2 r+1)} .
$$

Um die Berechnung zu beschleunigen, bietet es sich an, $\Phi(x)$ - bzw. im weiteren Verlauf $\eta_{\mathrm{ph}}$ - in einer Tabelle zu speichern, um daraus - ggf. durch Interpolation - die gesuchten Werte bestimmen zu können.

In Abbildung 4.3 ist der berechnete Verlauf der Photoionisationsrate sowohl nach dem vollständigen Keldysh-Modell (vgl. Abschnitt 4.2.1) als auch in den betrachteten Näherungen (vgl. Abschnitte 4.2.2 und 4.2.3) dargestellt. Man erkennt, dass für kleine Intensitäten - die nach Gleichung (4.4) einem großen Keldysh-Parameter $\gamma$ entsprechen - die vollständige Berechnung (rote Kurve) sehr gut sowohl mit Multiphotonennäherung von Keldysh als auch mit der von Kennedy verwendeten Näherung (vgl. Abschnitt 4.2.2) übereinstimmt. Diese beiden Näherungen sind für höhere Intensitäten nicht mehr gültig. Insbesondere liegen die aus der Berechnung der vollständigen Gleichung bestimmten Werte für die Ionisationsrate in diesem Fall deutlich unter den Werten der Kennedy-Näherung. Der zackenförmige Verlauf entsteht dadurch, dass die effektive Bandlücke mit steigender Intensität des eingestrahlten Lichts größer wird (vgl. Gleichung (4.3) ) und dadurch an bestimmten Stellen mehr Photonen für die Ionisation gebraucht werden, an denen es zu Einbrüchen der Ionisationsrate kommt. 


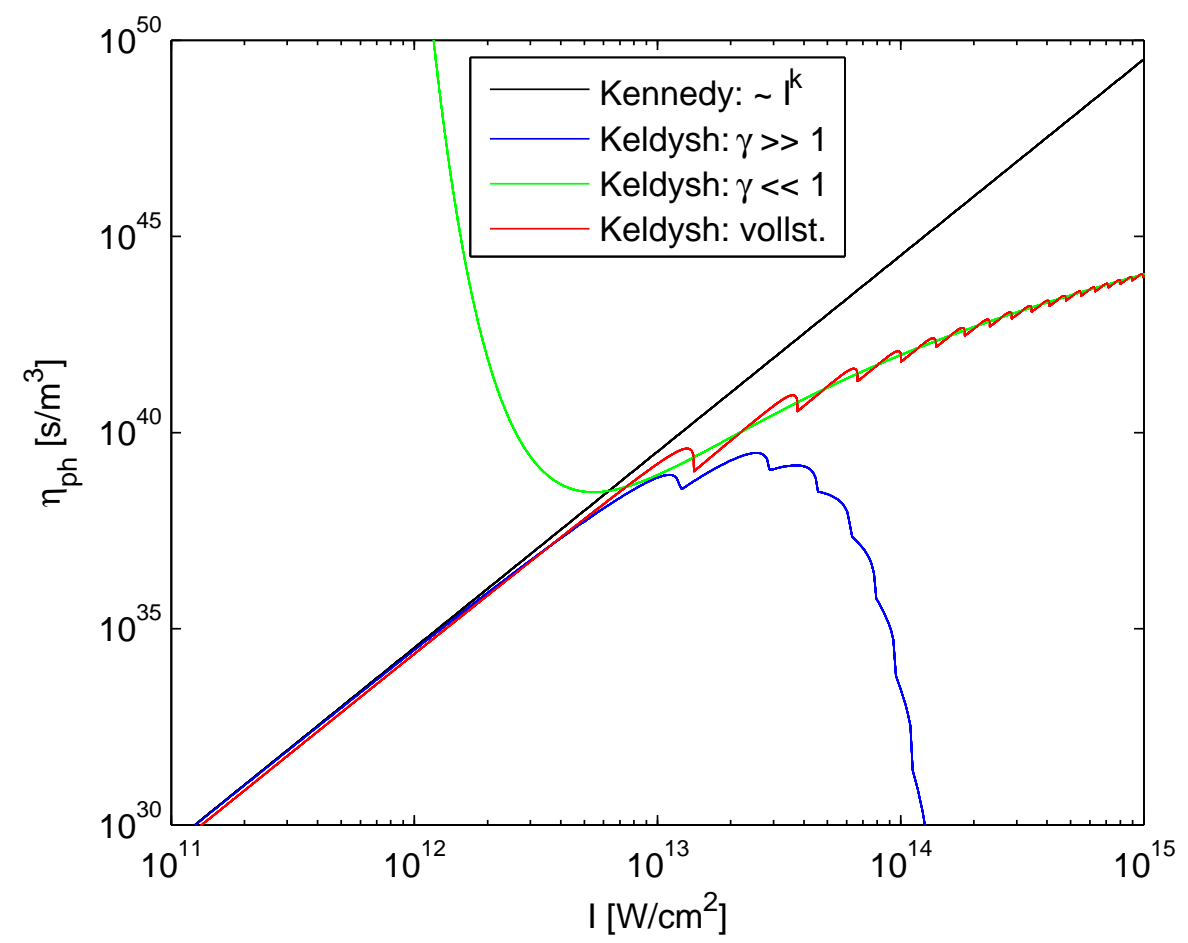

Abbildung 4.3: Verlauf der Rate für die Photoionisation nach dem Keldysh-Modell und nach dem von Kennedy [45] verwendeten Ansatz für die Multiphotonenabsorption $\eta_{\mathrm{ph}} \sim I^{\mathrm{k}}$.

Für $810 \mathrm{~nm}$ Wellenlänge ergibt sich aus Gleichung 4.4 eine Intensität $I \approx$ $1.76 \cdot 10^{13} \mathrm{~W} \mathrm{~cm}^{-2}$ für $\gamma=1$. Diese liegt im Bereich der zur Erzeugung eines optischen Durchbruchs nötigen Intensitäten (vgl. Abschnitt 4.7). In diesem Bereich wird die Näherung für die Multiphotonenabsorption zunehmend schlechter (vgl. Abbildung 4.3), so dass in den Rechnungen die vollständige Gleichung (4.1) verwendet wird.

In der von Keldysh angegebenen Rate wird nicht berücksichtigt, ob genug gebundene Elektronen vorhanden sind. Dies kann korrigiert werden, indem $\eta_{\mathrm{ph}}$ mit dem Verhältnis aus gebundenen und freien Elektronen multipliziert wird [80]. Man erhält damit für die Änderung der Elektronenzahldichte:

$$
\frac{\partial \rho}{\partial t}=\eta_{\mathrm{ph}} \frac{\rho-\rho_{\text {ges }}}{\rho_{\text {ges }}}=: \eta_{\mathrm{ph}}^{\prime}
$$

Dabei bezeichnet $\rho_{\text {ges }}$ die Dichte aller (für die Ionisierung relevanter) Elektronen im Ausgangszustand. Berücksichtigt man, dass beim Wassermolekül zwei Elektronen pro Molekül für die Ionisation in Frage kommen, so erhält man [45, 78, 79]:

$$
\rho_{\text {ges }}=2 \cdot 1.00 \frac{\mathrm{g}}{\mathrm{cm}^{3}} \cdot \frac{1 \mathrm{~mol}}{18.02 \mathrm{~g}} \cdot 6.02 \cdot 10^{23} \frac{1}{\mathrm{~mol}}=6.66 \cdot 10^{22} \frac{1}{\mathrm{~cm}^{3}} .
$$


Durch Gleichung (4.13) wird der Einfluss der Photoionisation auf die Elektronendichte beschrieben. Deren Auswirkung auf die Intensität des eingestrahlten Lichts wird im nächsten Abschnitt untersucht.

\subsubsection{Intensitätsabnahme und Entstehung freier Elektro- nen}

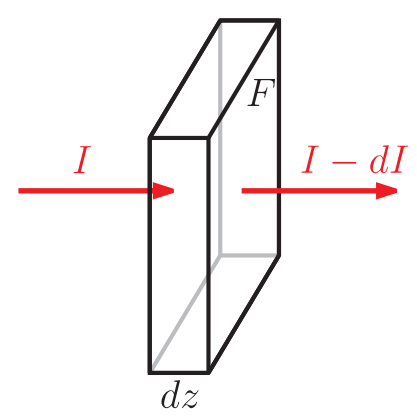

Abbildung 4.4: Zum Zusammenhang zwischen der Abnahme der Intensität und der Entstehung freier Elektronen

In diesem Abschnitt soll der Zusammenhang zwischen der Änderung der Intensität und der Dichte der freien Elektronen hergeleitet werden. Dazu betrachten wir einen Quader, in den Laserstrahlung senkrecht zu dessen Seitenfläche $F$ einfällt (vgl. Abbildung 4.4). Entlang der infinitesimalen Breite $d z$ nimmt die Intensität der Strahlung um $d I$ ab. Ist die Fläche $F$ klein genug gewählt, so dass die Intensität entlang der Fläche als konstant angenommen werden kann und bezeichnet $d t$ die Zeit, die das Licht braucht, um die Strecke $d z$ zurückzulegen, dann entspricht dies einer Abnahme der Energie von $F d I d t$. Die Energiedichte nimmt demnach um $d I d t / d z$ ab.

Die absorbierte Energie wird für die Ionisierung des Materials aufgewendet. Bezeichnet $\mathrm{E}_{\mathrm{ion}}$ die Energie, die benötigt wird, um ein freies Elektron zu erzeugen und $\rho$ die (Teilchen-)Dichte freier Elektronen, so entspricht eine Erhöhung der Dichte der freien Elektronen um $d \rho$ einer Änderung der Energiedichte von $\mathrm{E}_{\mathrm{ion}} d \rho$.

Setzt man die beiden berechneten Energiedichten gleich, so erhält man (in der Schreibweise partieller Ableitungen) [3]:

$$
\frac{\partial I}{\partial z}=-\mathrm{E}_{\mathrm{ion}} \frac{\partial \rho}{\partial t}
$$

Gleichung (4.15) stellt eine modifizierte Form des Lambert-Beerschen-Gesetzes dar [59]. 


\subsubsection{Bestimmung der Absorption bei Photoionisation}

Aus den Gleichungen (4.13) und (4.15) folgt für die Photoionisation mit der Einhüllenden der Intensität ohne den Einfluss des Plasmas $I_{0}$ :

$$
\begin{aligned}
-\mathrm{E}_{\mathrm{eff}} \eta_{\mathrm{ph}}^{\prime}=-\mathrm{E}_{\mathrm{eff}} \frac{\partial \rho}{\partial t} & =\frac{\partial I}{\partial z}=\frac{\partial}{\partial z}\left(I_{0} e^{2 i k_{\mathrm{ph}} z}\right)=2 i k_{\mathrm{ph}} I_{0} e^{2 i k_{\mathrm{ph}} z} \\
& =2 i \frac{\omega_{0}}{c}\left(\operatorname{Re}\left(n_{\mathrm{ph}}\right)+i \operatorname{Im}\left(n_{\mathrm{ph}}\right)\right) I \\
& =-2 \frac{\omega_{0}}{c} \operatorname{Im}\left(n_{\mathrm{ph}}\right) I+2 i \frac{\omega_{0}}{c} \operatorname{Re}\left(n_{\mathrm{ph}}\right) I .
\end{aligned}
$$

Man sieht sofort, dass $\operatorname{Re}\left(n_{\mathrm{ph}}\right)=0$ gelten muss. Gesucht ist nun ein Ausdruck für $\operatorname{Im}\left(n_{\mathrm{ph}}\right)$. Dazu wird der Brechnungsindex $n_{\text {ges }}$ betrachtet, der sowohl den Einfluss der linearen Polarisation $\mathbf{P}_{1}$ als auch den durch die Photoionisation bewirkten Anteil an der Polarisation $\mathbf{P}_{\mathrm{ph}}$ beschreibt. Unter der Annahme, dass $\chi_{\mathrm{ph}} \ll 1+\chi_{1}$ gilt, erhält man:

$$
\begin{aligned}
n_{\mathrm{ges}} & =\sqrt{\epsilon_{\mathrm{ges}}}=\sqrt{1+\chi_{\mathrm{ges}}}=\sqrt{1+\chi_{\mathrm{l}}+\chi_{\mathrm{ph}}}=\sqrt{\epsilon_{1}+\chi_{\mathrm{ph}}} \\
& \approx \sqrt{\epsilon_{1}}+\frac{1}{2} \frac{1}{\sqrt{\epsilon_{\mathrm{l}}}} \chi_{\mathrm{ph}}=n_{0}+\frac{1}{2 n_{0}} \operatorname{Re}\left(\chi_{\mathrm{ph}}\right)+\frac{i}{2 n_{0}} \operatorname{Im}\left(\chi_{\mathrm{ph}}\right) .
\end{aligned}
$$

Daraus ergibt sich unter Verwendung von Gleichung (4.16) für den Teil der Suszeptibilität, der durch die Photoionisation hervorgerufen wird:

$$
\chi_{\mathrm{ph}}=2 i n_{0} \operatorname{Im}\left(n_{\mathrm{ph}}\right)=i \frac{c n_{0}}{\omega_{0}} \frac{\mathrm{E}_{\mathrm{eff}} \eta_{\mathrm{ph}}^{\prime}}{I} .
$$

\subsection{Kaskadenionisation}

\subsubsection{Das Drude-Modell}

Im Folgenden wird die Wirkung eines äußeren elektrischen Feldes $\mathbf{E}=\mathbf{E}_{0} e^{-i \omega_{0} t}$ auf die (quasi-)freien Elektronen im Leitungsband untersucht [3, 48]. Nach dem Drude-Modell [23, 73] können die Elektronen (im Kristall) als freie Teilchen mit einer bestimmten effektiven Masse $m^{\star}$ beschrieben werden. Üblicherweise wird der Einfluss des Kristallgitters vernachlässigt und für die effektive Masse $m^{\star}$ die Masse von Elektronen im Vakuum verwendet $\left(m^{\star}=m_{e}\right)$. Als überwiegender Verursacher der Dämpfung werden Stöße der Elektronen mit den Gitteratomen angenommen, man spricht von einer Elektron-PhononStreuung. Sie kann durch eine Relaxationszeit $\tau_{\text {st }}$ beschrieben werden, die als mittlere Zeit zwischen zwei Stößen aufgefasst werden kann. Die Relaxationszeit liegt in der Größenordung von 1 fs [6]. In der vorliegenden Arbeit wird Vogel et al. [80] folgend der experimentell bestimmte Wert

$$
\tau_{\mathrm{st}}=1.7 \mathrm{fs}
$$


verwendet [75]. Der Ort x eines Elektrons kann durch die Bewegungsgleichung

$$
m^{\star} \frac{d^{2} \mathbf{x}}{d t^{2}}+m^{\star} \frac{1}{\tau_{\mathrm{st}}} \frac{d \mathbf{x}}{d t}=-e \mathbf{E}_{0} e^{-i \omega_{0} t}
$$

beschrieben werden. Für das eingeschwungene System ergibt sich als Lösung:

$$
\mathbf{x}=\frac{e}{m^{\star}} \frac{1}{\omega_{0}\left(\omega_{0}+\frac{i}{\tau_{\mathrm{st}}}\right)} \mathbf{E} .
$$

Aus der Verschiebung der Elektronen erhält man durch Multiplikation mit der Elektronenladung $-e$ deren Dipolmoment. Multipliziert man dieses wiederum mit der Dichte der Elektronen $\rho$, so erhält man die resultierende Polarisation des Mediums:

$$
\mathbf{P}_{\mathrm{st}}=-\rho e \mathbf{x}=\underbrace{-\frac{\rho e^{2}}{m^{\star}} \frac{\omega_{0} \tau_{\mathrm{st}}^{2}-i \tau_{\mathrm{st}}}{\omega_{0}\left(1+\omega_{0}^{2} \tau_{\mathrm{st}}^{2}\right)}}_{=\epsilon_{0} \chi_{\mathrm{st}}} \mathbf{E} .
$$

Durch diese wird der Brechungsindex des Materials geändert. Analog zur Gleichung (4.17) ergibt sich für den gesamten Brechungsindex:

$$
n_{\text {ges }}=n_{0}+\frac{1}{2 n_{0}} \operatorname{Re}\left(\chi_{\mathrm{st}}\right)+\frac{i}{2 n_{0}} \operatorname{Im}\left(\chi_{\mathrm{st}}\right) .
$$

Damit erhält man für den Imaginärteil von $n_{\text {st }}$ unter Verwendung der Gleichung (4.22):

$$
\begin{aligned}
\operatorname{Im}\left(n_{\mathrm{st}}\right) & =\frac{1}{2 n_{0}} \operatorname{Im}\left(\chi_{\mathrm{st}}\right) \\
& =\frac{\rho e^{2}}{2 n_{0} \epsilon_{0} m^{\star}} \cdot \frac{\tau_{\mathrm{st}}}{\omega_{0}\left(1+\omega_{0}^{2} \tau_{\mathrm{st}}^{2}\right)} \\
& =\frac{\sigma c \rho}{2 \omega_{0}} .
\end{aligned}
$$

Dabei bezeichnet $\sigma$ den Wirkungsquerschnitt der inversen Bremstrahlungsabsorption:

$$
\sigma=\frac{e^{2}}{n_{0} \epsilon_{0} m^{\star} c} \cdot \frac{\tau_{\mathrm{st}}}{1+\omega_{0}^{2} \tau_{\mathrm{st}}^{2}}
$$

In Analogie zur Gleichung (4.16) findet man:

$$
\mathrm{E}_{\mathrm{st}} \frac{\partial \rho}{\partial t}=2 \frac{\omega_{0}}{c} \operatorname{Im}\left(n_{\mathrm{st}}\right) I
$$

Dabei wird davon ausgegangen, dass die durch die inverse Bremsstrahlungsabsorption aufgenommene Energie sofort zum Erzeugen neuer freier Elektronen verwendet wird. $\mathrm{E}_{\mathrm{st}}$ ist die Energie, die für den Übergang eines Elektrons vom Valenzband ins Leitungsband bei einer Stoßionisation aufgebracht werden muss. Diese muss größer als die effektive Bandlücke $\mathrm{E}_{\text {eff }}$ sein, um die Energie- und Impulserhaltung im Stoß zu gewährleisten [80, 64]:

$$
\mathrm{E}_{\mathrm{st}}=\left(\frac{1+2 \mu}{1+\mu}\right) \mathrm{E}_{\mathrm{eff}} \quad \text { mit } \quad \mu=\frac{m_{e}}{m_{h}} .
$$


Werden $m_{e}$ und $m_{h}$ gleich gesetzt (vgl. Abschnitt 4.2.1), so ergibt sich:

$$
\mathrm{E}_{\mathrm{st}}=\frac{3}{2} \mathrm{E}_{\mathrm{eff}} \text {. }
$$

Aus den Gleichungen (4.26) und (4.24) erhält man für die Änderung der Elektronendichte durch die Kaskadenionisation:

$$
\frac{\partial \rho}{\partial t}=\eta_{\mathrm{st}} \rho \quad \text { mit } \quad \eta_{\mathrm{st}}=\frac{\sigma I}{\mathrm{E}_{\mathrm{st}}} .
$$

Elektronen können auch mit den Wassermolekülen zusammenstoßen, ohne ein Photon zu absorbieren. Dies kann berücksichtigt werden, indem $\eta_{\mathrm{st}}$ wie folgt erweitert wird [45, 80]:

$$
\eta_{\mathrm{st}}=\frac{1}{1+\omega_{0}^{2} \tau_{\mathrm{st}}^{2}}\left(\frac{e^{2} \tau_{\mathrm{st}}}{n_{0} \epsilon_{0} m^{\star} c \mathrm{E}_{\mathrm{st}}} I-\frac{m^{\star} \omega_{0}^{2} \tau_{\mathrm{st}}}{M}\right) .
$$

$M$ bezeichnet dabei die Masse eines Wassermoleküls, für die gilt:

$$
M=\frac{18.02 \mathrm{~g} / \mathrm{mol}}{6.02 \cdot 10^{23} 1 / \mathrm{mol}}=2.99 \cdot 10^{-22} \mathrm{~g} .
$$

In Gleichung (4.29) wird nicht berücksichtigt, dass die Kaskadenionisation eine bestimmte Zeit benötigt, in der sich bereits die Elektronendichte $\rho$ verändert haben kann. Dies kann näherungsweise durch Einfügen eines Korrekturfaktors [80] berichtigt werden. Es ergab sich aber kein bedeutender Einfluss auf die in Abschnitt 4.7 berechneten Elektronendichten.

Bei Berechnungen zum optischen Durchbruch wird das Drude-Modell häufig verwendet (vgl. 45], [27, Verweise in [3]). Alternativ kann für die Kaskadenionisation zum Beispiel ein von Rethfeld et al. [62, 63] vorgestelltes komplexeres Verfahren verwendet werden. Dieses berücksichtigt insbesondere, dass Elektronen unterschiedliche Energien besitzen können. Die bei der Anwendung dieses Modells benötigten Stoßwahrscheinlichkeiten für hochenergetische Elektronen können für Wasser allerdings nur sehr grob abgeschätzt werden. Zudem zeigt sich, dass - je nach Ansatz der Stoßwahrscheinlichkeiten - der Unterschied zwischen beiden Modellen nur gering ist [3]. Daher wird im Rahmen dieser Arbeit auf die Anwendung eines solchen Modells verzichtet.

\subsubsection{Plasmafrequenz und kritische Dichte}

Nimmt man an, dass $\omega_{0} \gg 1 / \tau_{\text {st }}$ gilt, so kann der Imaginärteil der Polarisation in Gleichung (4.22) vernachlässigt werden. Für die Dielektrizitätskonstante ergibt sich [48]:

$$
\epsilon_{\mathrm{ges}}=\epsilon_{1}+\chi_{\mathrm{st}}=\epsilon_{\mathrm{l}}\left(1-\frac{\chi_{\mathrm{st}}}{\epsilon_{1}}\right)=\epsilon_{1}\left(1-\frac{\omega_{\mathrm{P}}}{\omega_{0}}\right) \quad \text { mit } \quad \omega_{\mathrm{P}}^{2}=\frac{\rho e^{2}}{\epsilon_{0} \epsilon_{1} m^{\star}} .
$$


Dabei bezeichnet die neu eingeführte Größe $\omega_{\mathrm{p}}$ die sogenannte Plasmafrequenz 81. Man erkennt, dass für $\omega_{\mathrm{p}}>\omega_{0}$ die Dielektrizitätskonstante $\epsilon_{\text {ges }}$ negativ wird. Dies führt dazu, dass der Brechnungsindex (rein) imaginär wird.

Für das Reflexionsvermögen $R_{12}$ an der Grenze zwischen zwei Materialien mit den Brechungsindizes $n^{(1)}$ und $n^{(2)}$, wobei $n^{(1)}$ reell angenommen wird und $n^{(2)}=n_{r}+i \cdot n_{i}$ gilt, findet man für senkrecht einfallendes Licht [59]:

$$
R_{12}=\left(\frac{n^{(1)}-n^{(2)}}{n^{(1)}+n^{(2)}}\right)^{2}=\frac{\left(n_{r}-n^{(1)}\right)^{2}+n_{i}^{2}}{\left(n_{r}+n^{(1)}\right)^{2}+n_{i}^{2}}
$$

Für einen rein imaginären Brechungsindex $n^{(2)}$ erhält man mit $R_{12}=1$ Totalreflexion. Es existiert daher eine kritische Dichte $\rho_{\mathrm{cr}}$, ab der das Plasma reflektierend wirkt. Aus Gleichung (4.32) erhält man:

$$
\rho_{\mathrm{cr}}=\omega_{0}^{2} \frac{\epsilon_{0} \epsilon_{1} m^{\star}}{e^{2}}
$$

Für die vorwiegend in dieser Arbeit verwendete Laserwellenlänge $\lambda=810 \mathrm{~nm}$ $\left(\omega_{0} \approx 3.1 \mathrm{fs}^{-1}\right)$ findet $\operatorname{man} \rho_{\mathrm{cr}} \approx 3 \cdot 10^{21} \mathrm{~cm}^{-3}$.

Die angenommene Näherung $\omega_{0} \gg 1 / \tau_{\text {st }}$ wird oft bei der Betrachtung von Metallen verwendet, bei denen die Stoßzeit $\tau_{\text {st }}$ größer als in Halbleitern ist. Für Wasser und Laserstrahlung im sichtbaren bzw. nahen infraroten Bereich ist sie nur schwach erfüllt, für Strahlung der Wellenlänge $\lambda=810 \mathrm{~nm}$ erhält man $\omega_{0} \approx 5.3 / \tau_{\text {st }}$. Daher wird die Näherung nur zur Abschätzung der kritischen Dichte verwendet.

Bei der gekoppelten Berechnung von Pulsausbreitung und Plasmabildung (s. Kapite1 5) muss darauf geachtet werden, dass die auftretenden Elektronendichten die kritische Dichte deutlich unterschreiten, da bei der Lösung der Einhüllendengleichung keine zurücklaufenden Wellen berücksichtigt werden können (vgl. Abschnitt 1.5.2).

\subsection{Diffusion und Rekombination}

Die Bewegung der Elektronen im Leitungsband kann durch eine Diffusionsgleichung beschrieben werden:

$$
\frac{\partial \rho}{\partial t}=-D \cdot \triangle \rho
$$

Dabei wird die Diffusionskonstante $D$ im einfachsten Fall als isotrop und unabhängig von der Temperatur angenommen. Bezeichnet $\mathrm{E}_{\text {avg }}$ die durchschnittliche Energie der Elektronen, so erhält man [45]:

$$
D=\frac{2}{3} \frac{\mathrm{E}_{\mathrm{avg}} \tau_{\mathrm{st}}}{m_{e}} .
$$


Berücksichtigt man, dass Elektronen eine Energie von $2 \mathrm{E}_{\text {eff }}$ benötigen, um durch Stoßionisation ein neues freies Elektron der Energie $E_{\text {eff }} z u$ erzeugen, so ergibt sich:

$$
\mathrm{E}_{\mathrm{avg}}=\frac{3}{2} \mathrm{E}_{\mathrm{eff}} \text {. }
$$

Die Diffusionsgleichung (4.35) kann unter Verwendung des Laplace-Operators in Zylinderkoordinaten ebenfalls mit dem Crank-Nicolson-Verfahren (vgl. Abschnitt 3.3.3) gelöst werden.

Interessiert man sich nur für den Einfluss der Diffusion auf die Maximaldichte, so kann anstelle der Diffusionsgleichung die folgende Gleichung

$$
\frac{\partial \rho}{\partial t}=-\eta_{\text {diff }} \rho, \quad \eta_{\text {diff }}=\frac{D}{L_{\text {diff }}^{2}} .
$$

verwendet werden [45]. $L_{\text {diff }}$ bezeichnet dabei die Diffusionslänge, für ein zylindrisches Fokusvolumen mit der Länge $l$ und dem Durchmesser $d$ ergibt sich 45]:

$$
\frac{1}{L_{\mathrm{diff}}^{2}}=\left(\frac{4.8}{d}\right)^{2}+\left(\frac{1}{l}\right)^{2} .
$$

Man muss dabei allerdings beachten, dass Gleichung (4.38) nur eine Näherung der Diffusionsgleichung darstellt. So erhält man - im Gegensatz zur Diffusionsgleichung - einen exponentiellen Abfall der Maximaldichte.

Neben der Diffusion trägt die Rekombination zu einer Reduktion der (maximalen) Elektronendichte bei. Die Rekombinationsrate der Elektronen wird mit

$$
\eta_{\mathrm{rec}}=2 \cdot 10^{-9} \frac{\mathrm{cm}^{3}}{\mathrm{~s}}
$$

angenommen. Dieser Wert wurde experimentell von Docchio [22] über den Abfall der Plasmalumineszenz bestimmt.

\subsection{Ratengleichung}

In den vorangegangenen Abschnitten wurde die Änderung der Elektronendichte durch Photonenionisation, Kaskadenionisation, Diffusion und Rekombination einzeln betrachtet. Der Einfluss dieser Effekte kann in der folgenden Ratengleichung zusammengefasst werden [58, 45, 46]:

$$
\frac{d \rho}{d t}=\eta_{\mathrm{ph}} \frac{\rho-\rho_{\text {ges }}}{\rho_{\text {ges }}}+\eta_{\text {st }} \rho-\eta_{\text {diff }} \rho-\eta_{\text {rec }} \rho^{2} .
$$

Bei Vernachlässigung des Diffusionsterms $-\eta_{\text {diff }} \rho$ gilt Gleichung (4.41) für die Elektronendichte an allen Orten. Wie in Abschnitt 4.4 beschrieben, kann die Diffusion nur für die Maximaldichte in dieser Form berechnet werden, zur Berechnung der Elektronendichte an einem beliebigen Ort muss die Diffusionsgleichung (4.35) gelöst werden. 


\subsection{Durchbruchsschwelle}

Im Experiment wird das Auftreten des optischen Durchbruchs in Flüssigkeiten häufig über das Auftreten einer Kavitationsblase (vgl. Abschnitt 4.1) definiert. Dies entspricht in Festkörpern einer nachhaltigen Modifikation des Materials [69].

Alternativ kann der optische Durchbruch durch das Leuchten des entstehenden Plasmas nachgewiesen werden. Dies ist bei Pulsen von wenigen Nanosekunden Dauer oft mit dem bloßen Auge möglich. Bei fs-Pulsen ist die Plasmalumineszenz allerdings deutlich geringer, so dass das Auftreten einer Blase einen einfacheren Nachweis für den optischen Durchbruch darstellt.

Die kleinste Pulsenergie (oder Intensität), für die gerade ein optischer Durchbruch (mit einer gewissen Wahrscheinlichkeit) stattfindet, definiert die Durchbruchsschwelle.

Im theoretischen Arbeiten wird diese im Allgemeinen durch das Erreichen einer bestimmten Elektronendichte $\rho_{\text {thr }}$ bestimmt. Für deren Größe findet man in der Literatur stark unterschiedliche Werte.

So gibt Kennedy [45] zum einen den von ihm als „bubble endpoint“ bezeichneten Wert $\rho_{\mathrm{thr}}=10^{18} \mathrm{~cm}^{-3}$ an, der durch die Blasenentstehung motiviert ist und ungefähr dem Beginn einer signifikanten Absorption durch das Plasma entspricht. Zum anderen definiert er den ,flash endpoint" mit $\rho_{\text {thr }}=10^{20} \mathrm{~cm}^{-3}$. Dieser Wert wurde aus der Vorraussetzung abgeschätzt, dass das Plasma eine Temperatur von $15000 \mathrm{~K}$ haben soll. Vogel et al. [80] nehmen eine noch größere Elektronenzahldichte an, die mit $\rho_{\mathrm{thr}}=10^{21} \mathrm{~cm}^{-3}$ in der Größenordnung der kritischen Dichte (vgl. Abschnitt 4.3.2) für typische Laserwellenlängen liegt.

\subsection{Beispiele}

Bei bekannter Intensität stellt die Ratengleichung (4.41) eine gewöhnliche Differentialgleichung dar, die mit einem schrittweitengesteuerten Runge-KuttaVerfahren 61] gelöst werden kann [58]. Die Intensität kann dabei zum Beispiel durch ein zeitliches Gauß-Profil (1.29) oder sech-Profil (1.28) vorgeben werden.

Im Folgenden wird der zeitliche Verlauf der Elektronendichte für einen Puls mit einer Dauer von $130 \mathrm{fs}$ (vgl. Abbildung 4.5) vorgestellt und mit den Ergebnissen für einen ns-Puls (vgl. Abbildung 4.6) verglichen. In beiden Abbildungen ist jeweils das Ergebnis für die komplette Ratengleichung (blaue Kurve) und die Elektronendichte bei vernachlässigter Kaskadenionisation dargestellt.

Im Fall des fs-Pulses erkennt man einen stetigen, glatten Anstieg der Elektronendichte bis nach Durchlaufen des Pulses die maximale Dichte erreicht ist. Der Abfall der Elektronendichte hinter dem Puls ist auf der betrachteten 


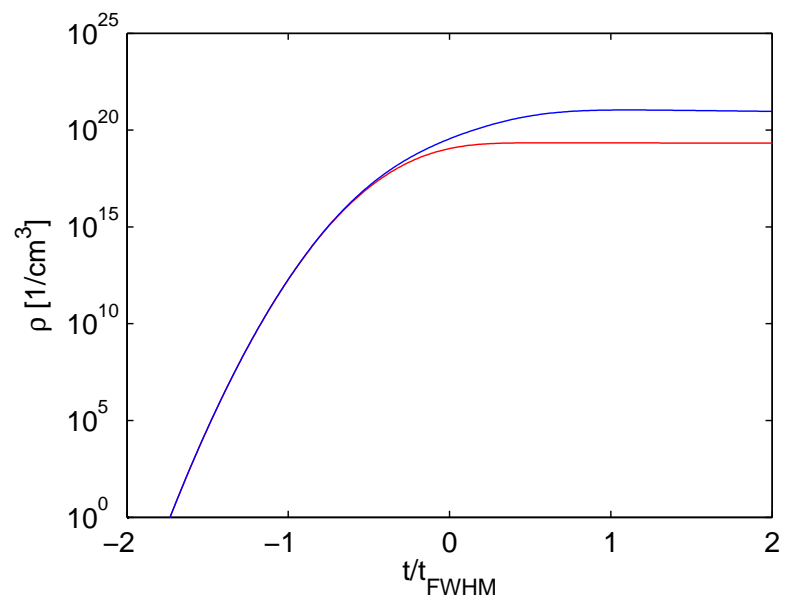

Abbildung 4.5: Berechnete Elektronendichte für einen fs-Laserpuls
$\left(t_{\mathrm{FWHM}}=130 \mathrm{fs}, \lambda=800 \mathrm{~nm}, I_{0}=7.8 \cdot 10^{11} \mathrm{~W} / \mathrm{cm}^{2}\right)$. Die In-
tensität wurde so gewählt, dass die Elektronendichte den Schwellwert
$\rho_{\text {thr }}=10^{21} \mathrm{~cm}^{-3}$ erreicht [80]. Die blaue Kurve stellt das Ergebnis der
kompletten Ratengleichung dar, während bei der roten Kurve nur die
Photoionisation als Mechanismus der Plasmabildung berücksichtigt wird.

Zeitskala sehr gering. Daher können bei den Berechnungen in Kapitel 5 die Diffusion und die Rekombination vernachlässigt werden.

Ferner ist in Abbildung 4.5 zu sehen, dass im Fall eines fs-Pulses die Photoionisation einen sehr großen Anteil an der Entstehung von freien Elektronen hat, der Einfluss der Kaskadenionisation aber nicht vernachlässigt werden kann.

Abbildung 4.6 zeigt für einen ns-Puls einen deutlich anderen Verlauf der Elektronendichte. Auffällig ist der steile Anstieg der Dichte in der Mitte des Pulses. Dieser kommt daher, dass für den Start der Kaskadenionisation vorausgesetzt wurde, dass mindestens ein Elektron mit einer Wahrscheinlichkeit von 50\% im Fokusvolumen vorhanden ist. Ist die entsprechende Elektronendichte erreicht, so werden durch die Kaskadenionisation sehr schnell neue freie Elektronen erzeugt, bis vor allem die Rekombination diesen Prozess aufhält. Für das Fokusvolumen wurde ein Ellipsoid mit dem Durchmesser $1800 \mathrm{~nm}$ auf der Rotationsachse und $750 \mathrm{~nm}$ senkrecht dazu gewählt [80]. Es zeigt sich, dass es bei der Betrachtung von fs-Pulsen nicht erforderlich ist, eine Begrenzung für den Start der Kaskadenionisation einzufügen.

Ein weiterer Unterschied beim Vergleich der beiden Pulse besteht darin, dass bei fs-Pulsen die erreichte maximale Elektronendichte stetig von der Intensität des betrachteten Pulses abhängt. So ergibt sich bei einer für den optischen Durchbruch angenommenen Elektronendichte von $\rho_{\mathrm{thr}}=10^{20} \mathrm{~cm}^{-3}$ bzw. $\rho_{\mathrm{thr}}=$ $10^{18} \mathrm{~cm}^{-3}$ eine nötige Intensität von $I_{0}=5.8 \cdot 10^{11} \mathrm{~W} / \mathrm{cm}^{2}$ bzw. $I_{0}=3.1$. $10^{11} \mathrm{~W} / \mathrm{cm}^{2}$. Bei ns-Pulsen ist dies nicht der Fall, da die Intensität nur dafür ausreichen muss, die Erzeugung eines Elektrons durch Photonenionisation zu gewährleisten. Der daraufhin einsetzenden Kaskadenionisation steht genügend 


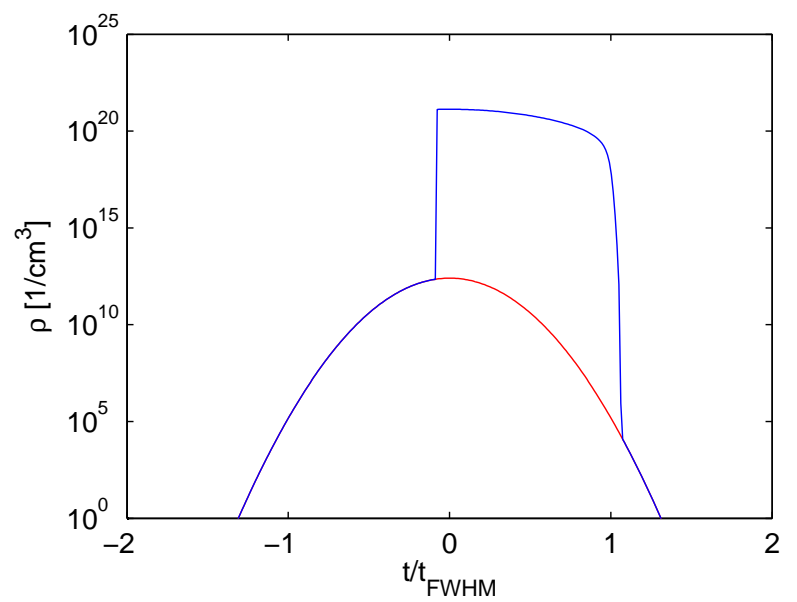

Abbildung 4.6: Elektronendichte für einen ns-Laserpuls mit $t_{\mathrm{FWHM}}=$ $6 \mathrm{~ns}, \lambda=1000 \mathrm{~nm}$ und $I_{0}=1.9 \cdot 10^{11} \mathrm{~W} / \mathrm{cm}^{2}$ (vgl. Abbildung 4.5).

Energie zur Verfügung, um Elektronendichten im Bereich der kritischen Dichte zu erzeugen.

Die Parameter der betrachteten Pulse wurden den Berechnungen von Vogel et al. 80] angepasst, um die Rechnungen vergleichen zu können. Dabei ergibt sich eine sehr gute Übereinstimmung im Kurvenverlauf und bei den nötigen Intensitäten. Lediglich die Kurve für den Verlauf der Elektronendichte bei Vernachlässigung der Kaskadenionisation zeigt Abweichungen. 


\section{Kapitel 5}

\section{Pulsausbreitung und Plasmadynamik}

In diesem Kapitel wird beschrieben, wie die Berechnung der Pulsausbreitung mit der des Plasmas kombiniert werden kann. Dazu wird insbesondere die Ausbreitungsgleichung um den Einfluss des Plasmas erweitert. Es wird eine Reihe von Ergebnissen dieser Berechnungen vorgestellt und diskutiert. Dabei steht der Vergleich mit experimentellen Beobachtungen im Vordergrund.

\section{$5.1 \quad$ Erweiterte Ausbreitungsgleichung}

Bisher wurde in der Einhüllendengleichung (1.50) nur der Anteil an der nichtlinearen Polarisation, der durch den Kerr-Effekt bewirkt wird, berücksichtigt. $\mathrm{Zu}$ diesem treten die durch das Plasma hervorgerufene Änderungen der Polarisation hinzu. Für die Photoionisation wurde diese in Gleichung (4.18) wie folgt bestimmt:

$$
\mathbf{P}_{\mathrm{ph}}=i \frac{c n_{0} \epsilon_{0}}{\omega_{0}} \frac{\mathrm{E}_{\mathrm{eff}} \eta_{\mathrm{ph}}^{\prime}}{I} \mathbf{E} .
$$

Ferner ergibt sich für den durch die Stöße der Elektronen mit den Molekülrümpfen bewirkten Anteil an der nichtlinearen Polarisation nach Gleichung (4.22) mit den Gleichungen (4.25) und (1.27):

$$
\mathbf{P}_{\mathrm{st}}=-\frac{\rho \sigma n_{0}^{2} \epsilon_{0}}{\beta_{0}}\left(\omega_{0} \tau_{\mathrm{st}}-i\right) \mathbf{E} .
$$

Addiert man die hinzugekommenen Polarisationsanteile (5.2) und (5.1) zu der durch den Kerr-Effekt bewirkten Polarisation (1.17) und erweitert die Definition der Einhüllenden der nichtlinearen Polarisation $B$ in Gleichung (1.35) 
entsprechend, erhält man - bei Vernachlässigung der zeitlichen Ableitung von $B$ - als zusätzliche Terme zur Einhüllendengleichung (1.51):

$$
\partial_{\xi} A=\ldots+i \frac{\mu_{0} \omega_{0}^{2}}{2 \beta_{0}}\left(i \frac{c n_{0} \epsilon_{0}}{\omega_{0}} \frac{\mathrm{E}_{\mathrm{eff}} \eta_{\mathrm{ph}}^{\prime}}{I} A-\frac{\rho \sigma n_{0}^{2} \epsilon_{0}}{\beta_{0}}\left(\omega_{0} \tau_{\mathrm{st}}-i\right) A\right) .
$$

Durch Verwendung der Gleichungen (1.22), (1.32) und (1.27) ergibt sich:

$$
\partial_{\xi} A=\ldots-\frac{1}{\epsilon_{0} c n_{0}} \frac{\mathrm{E}_{\mathrm{eff}} \eta_{\mathrm{ph}}^{\prime}}{|A|^{2}} A-\frac{\sigma}{2}\left(1+i \omega_{0} \tau_{\mathrm{st}}\right) \rho A
$$

Für die im Rahmen dieser Arbeit durchgeführten Simulationen ist die Ausbreitungsgleichung nun vollständig. Wie in Abschnitt 2.4 beschrieben, kann der für die Pulsaufsteilung verantwortliche Term bei den betrachteten Pulsen mit Längen um 100 fs und mehr ebenso wie der Einfluss der linearen Absorption (im Bereich des Fokus) vernachlässigt werden. Man erhält:

$$
\begin{aligned}
\partial_{\xi} A= & i \hat{D} A & & \text { (Dispersion) } \\
& +\frac{i}{2 \beta_{0}}\left(1+\frac{i}{\omega_{0}} \partial_{\tau}\right)^{-1} \triangle_{\perp} A & & \text { (Beugung) } \\
& +i \frac{\beta_{0} c \epsilon_{0}}{2} n_{2}|A|^{2} A & & \text { (Kerr-Nichtlinearität) } \\
& -\frac{1}{\epsilon_{0} c n_{0}} \frac{\mathrm{E}_{\mathrm{eff}} \eta_{\mathrm{ph}}^{\prime}}{|A|^{2}} A & & \text { (Absorption durch Photoionisation) } \\
& -\frac{\sigma}{2} \rho A & & \text { (Absorption durch Stöße) } \\
& -\frac{\sigma}{2} i \omega_{0} \tau_{\mathrm{st}} \rho A . & & \text { (Defokussierung durch Stöße) }
\end{aligned}
$$

In Abschnitt 2.4 wurde gezeigt, dass der Term der Kerr-Nichtlinearität zusammen mit dem Beugungsterm zur Selbstfokussierung führt. Der letzte Term in Gleichung (5.5) ist wie der Term der Kerr-Nichtlinearität rein imaginär, besitzt aber ein negatives Vorzeichen. Dieses führt in Verbindung mit dem für die Beugung verantwortlichen Term zu einer Defokussierung des Pulses.

\subsection{Numerisches Verfahren}

Wie in Abschnitt 3.3 beschrieben, wird zunächst die Pulsform für einen Ort vor dem Fokus bestimmt (vgl. Abschnitt 3.3.1), an dem angenommen werden kann, dass die Intensität für das Ablaufen der nichtlinearen Prozesse einschließlich der Plasmabildung noch nicht ausreichend ist.

$\mathrm{Ab}$ diesem Punkt wird die Pulsausbreitung durch das Lösen der Ausbreitungsgleichung (5.5) mit Hilfe des in Abschnitt (2.1) beschriebenen SplitOperator-Verfahrens berechnet. Die in Gleichung (5.5) durch die Berücksichtigung der Plasmabildung hinzugekommenen Terme können dabei analog zur 
Kerr-Nichtlinearität berechnet werden, indem der Operator $\hat{N}$ in Gleichung (2.3) entsprechend erweitert wird.

Dazu muss die Dichte der freien Elektronen vor jedem Schritt bestimmt werden. Dies geschieht durch Lösung der Ratengleichung (4.41) mit einem schrittweitengesteuerten Runge-Kutta-Verfahren (vgl. Abschnitt 4.7) für alle Werte der Diskretisierung in $r$-Richtung. Der für die Berechnung erforderliche Intensitätsverlauf wird dabei wenn nötig durch Interpolation bestimmt.

\subsection{Aufbau des Vergleichsexperiments}

Die Ergebnisse der Berechnungen zur Pulsausbreitung unter Berücksichtigung der Plasmabildung sollen mit experimentellen Daten zum optischen Durchbruch in Wasser von Kröninger [49] verglichen werden können. Daher werden die Parameter der Simulation an die Bedingungen des Experiments angepasst. Im Folgenden wird der experimentelle Aufbau skizziert.

Im Experiment kommt ein Femtosekundenlasersystem zu Einsatz, dessen Pulse eine Pulsdauer von $t_{\mathrm{FWHM}}=130$ fs (bei Gaußförmigem Verlauf) und eine Wellenlänge von ca. $\lambda=810 \mathrm{~nm}$ besitzen. Für das Strahlprofil wird ein GaußProfil mit einem Strahlradius von $2 \mathrm{~mm}$ angenommen.

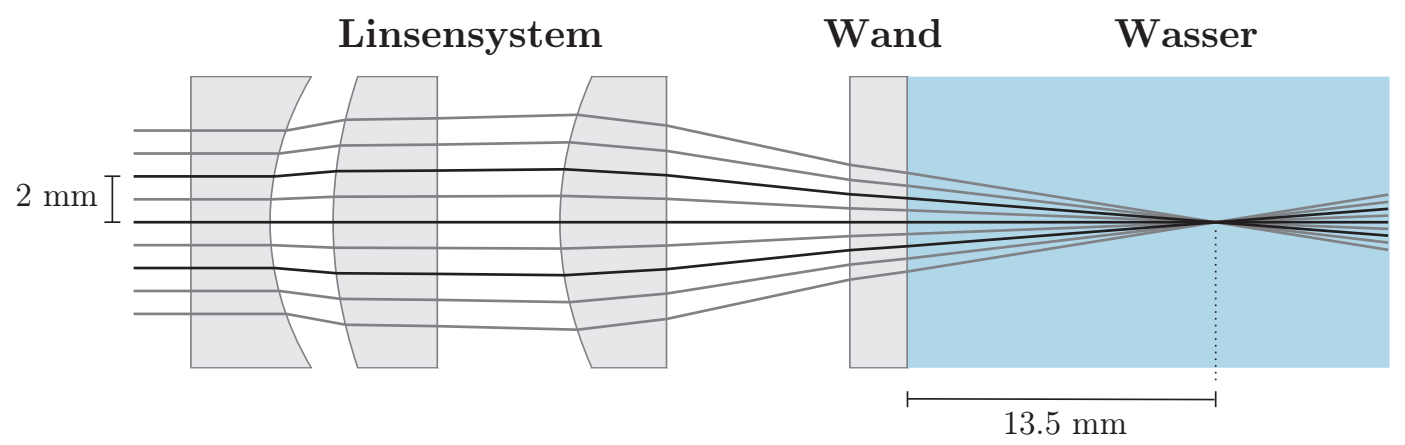

Abbildung 5.1: Strahlengang des zum Vergleich herangezogenen Experiments [49].

Die Laserpulse werden mit Hilfe eines Linsensystems in eine mit Wasser gefüllte Küvette fokussiert. Wie in Abschnitt 3.1.6 beschrieben, besteht der größte Linsenfehler bei der Fokussierung in eine wassergefüllte Küvette in der sphärischen Aberration. Deshalb wurde ein Linsensystem verwendet, bei dem diese mit Hilfe eines Raytracing-Programms von Geisler [33] durch Variation der Position der ersten Linse minimiert wurde. Abbildung 5.1 zeigt das Linsensystem und den durch das Programm berechneten Strahlengang. Die hervorgehobenen Strahlen sind im Abstand des Strahlradius von der optischen Achse berechnet. 
An der Innenwand der Küvette beträgt der Abstand dieser Strahlen zur optischen Achse $w_{0}=1.04 \mathrm{~mm}$. Die Strecke zwischen der Küvettenwand und dem Fokus beträgt $f=13.5 \mathrm{~mm}$. Damit ergibt sich eine numerische Apertur von NA $\approx 0.1$. Für die Strahltaille (eines Pulses mit Gauß-Profil) erhält man $w_{\mathrm{f}}=2.52 \mu \mathrm{m}$.

Die Pulsenergiebestimmung erfolgte mit Hilfe einer Photodiode vor dem Linsensystem. Die Energie des Pulses kurz vor dem Fokus wurde bestimmt, indem die Transmission aus der Reflexion an den Grenzflächen und der Absorption im Glas und Wasser berechnet wurde. Es ergab sich eine Transmission von $67.4 \%$ [9].

\subsection{Ergebnisse der Simulation}

Abbildung 5.2 zeigt die mit den in Abschnitt 5.3 diskutierten Parametern berechnete Elektronendichte für unterschiedliche Laserpulsenergien.

Dabei ist zu beachten, dass die Skalierung der Achsen unterschiedlich ist. Man erkennt sofort, dass die entstehenden Plasmen deutlich stärker entlang der optischen Achse als in $r$-Richtung ausgedehnt sind. Die Größe der Plasmen nimmt bei steigender Energie zu, wobei sie sich überwiegend in die Richtung ausdehnen, aus der der Puls gekommen ist.

Die maximale Elektronendichte im Plasma steigt zunächst mit der Energie an, bis sie für $\mathrm{E}=0.2 \mu \mathrm{J}$ einen Wert von $\rho_{\max }=7.28 \cdot 10^{19} \mathrm{~cm}^{-3}$ erreicht. Für höhere Energien reduziert sich der Wert für die maximale Elektronendichte wieder.

Für die Pulsenergie $\mathrm{E}=0.4 \mu \mathrm{J}$ sind in Abbildung 5.3 die Pulsformen für verschiedene $z$-Werte dargestellt. Zunächst besitzt der Puls einen in zeitlicher und räumlicher Richtung gaußförmigen Verlauf. Mit zunehmender Plasmabildung (vgl. Abbildung 5.2) beginnt die Intensität für den (zeitlich) hinteren Teil des Pulses abzunehmen und dieser Teil immer weiter auseinanderzulaufen. Im weiteren Verlauf ist nur noch der vordere Teil des Pulses vorhanden, der sich nach Durchlaufen des Fokus wieder ausdehnt.

Das „Aushöhlen“ des Pulses und die Ausdehnung in transversaler Richtung werden durch die Kombination aus Absorption und Defokussierung des Plasmas (vgl. Abschnitt 5.1) bewirkt. Den Einfluss dieser beiden Effekte zeigt Abbildung 5.4, die die Ergebnisse von Elektronendichteberechnungen darstellt, bei denen jeweils der Einfluss der Defokussierung oder der Absorption vernachlässigt wurde. Man erkennt insbesondere bei einem Vergleich der vollständigen Rechnung mit der Simulation ohne Defokussierung einen sehr deutlichen Unterschied in der Größe und Form des entstehenden Plasmas. Fehlt der defokussierende Effekt, so schnürt sich das Plasma noch enger zusammen und die Dichte ist - für die gewählte Pulsenergie $\mathrm{E}=0.5 \mu \mathrm{J}$ - um ungefähr den Faktor 

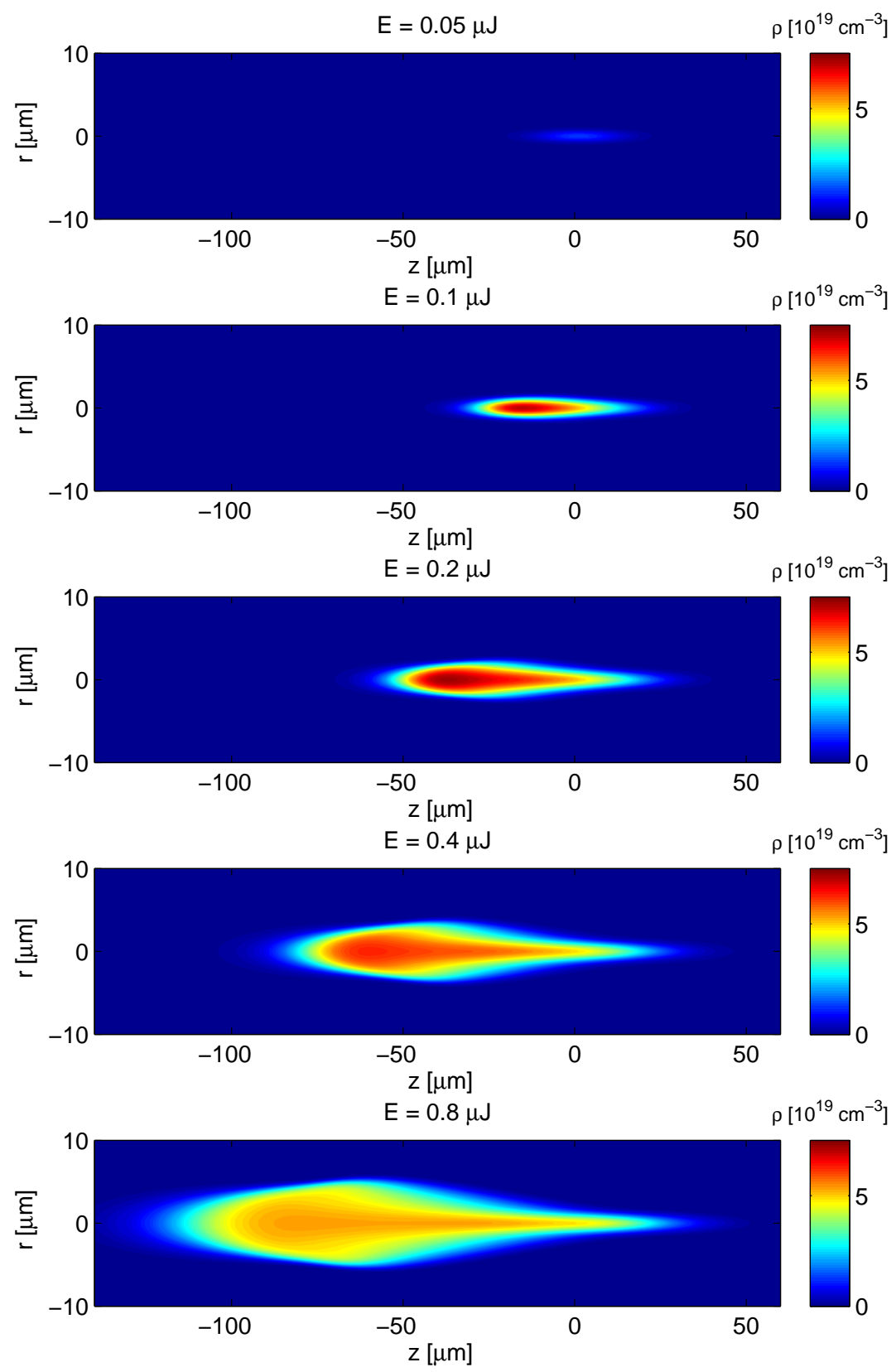

Abbildung 5.2: Berechnete Elektronendichte für unterschiedliche Pulsenergien zwischen mit den Parametern aus Abschnitt 5.3. Die Grafiken wurden für eine bessere Darstellung um den Faktor 2.27 gestaucht. Am Ort $z=0$ liegt der Fokus bei linearer Pulsausbreitung.

13 höher. Bei der Berechnung ohne Absorption fällt der Unterschied in der Pulsform nicht so groß aus, die maximale Elektronendichte ist aber auch hier deutlich größer.

In Abbildung 5.5 ist der Verlauf der (über die transversalen Richtungen integrierten) Elektronendichte in Abhängigkeit von $z$ für unterschiedliche Energien 

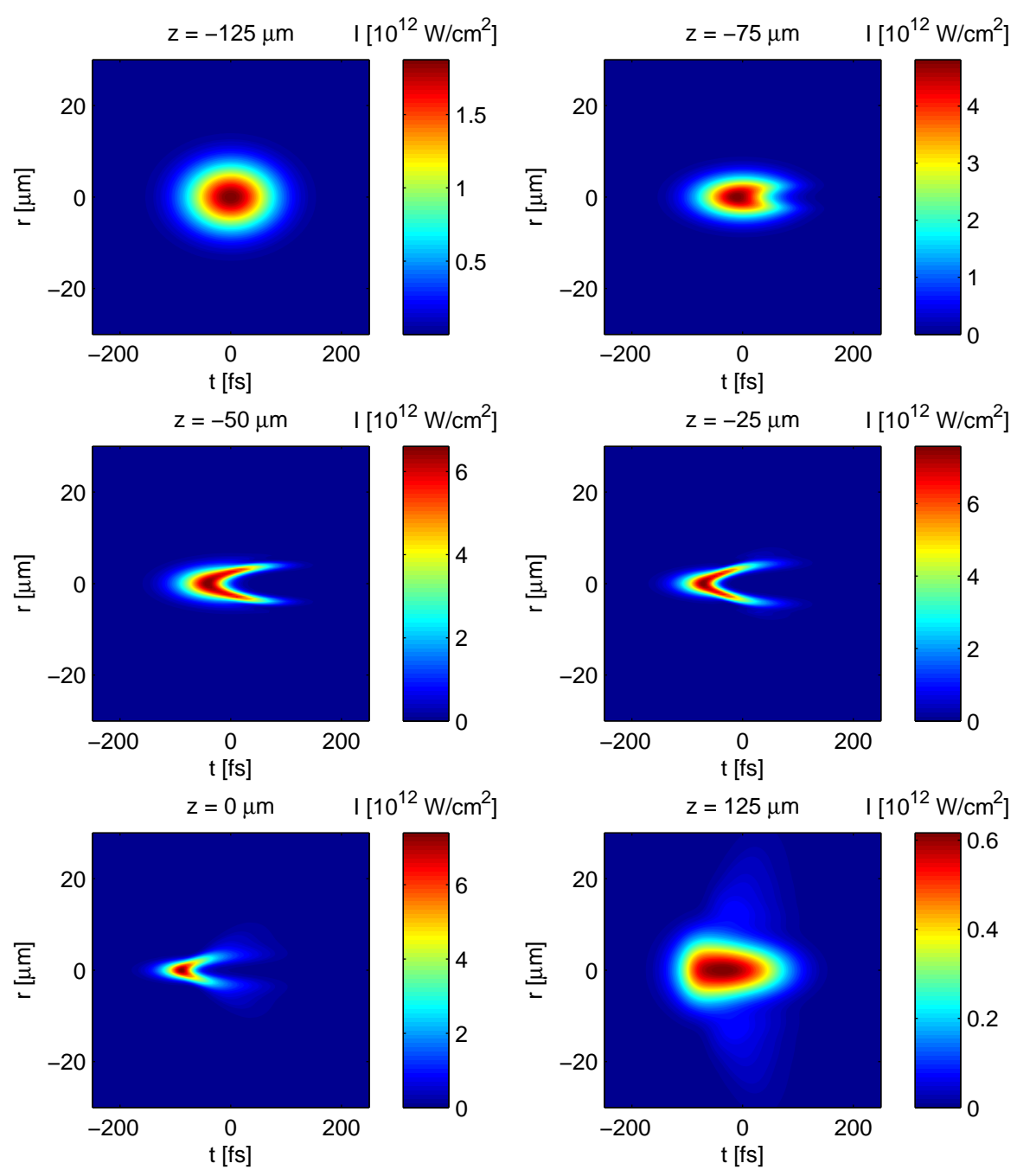

Abbildung 5.3: Berechneter Intensitätsverlauf für unterschiedliche $z$, $\mathrm{E}=0.4 \mu \mathrm{J}$. Zur besseren Darstellung wurden unterschiedliche Farbskalen gewählt.

dargestellt. Man erkennt, dass alle Kurven einen relativ symmetrischen Verlauf zeigen, obwohl die Formen der Plasmen (vgl. Abbildung 5.2) diese Eigenschaft nicht aufweisen. Ferner ist zu sehen, dass die (integrierte) Dichte für steigende Energie an einem immer kleineren Wert für $z$ maximal wird.

Durch Integration der Elektronendichte über das betrachtete Volumen kann man die Anzahl der freien Elektronen erhalten. Multipliziert man diese mit der mittleren Energie eines Elektrons (vgl. Gleichung (4.37)), so erhält man die im Plasma enthaltende Energie. Da bei den Berechnungen die Diffusion und Rekombination vernachlässigt wurden, entspricht diese der absorbierten Energie. Deren Verhältnis zu der (initialen) Pulsenergie ist in Abbildung 5.6 dargestellt. Wie man sieht, steigt dieses mit wachsender Energie des Pulses stark an. 

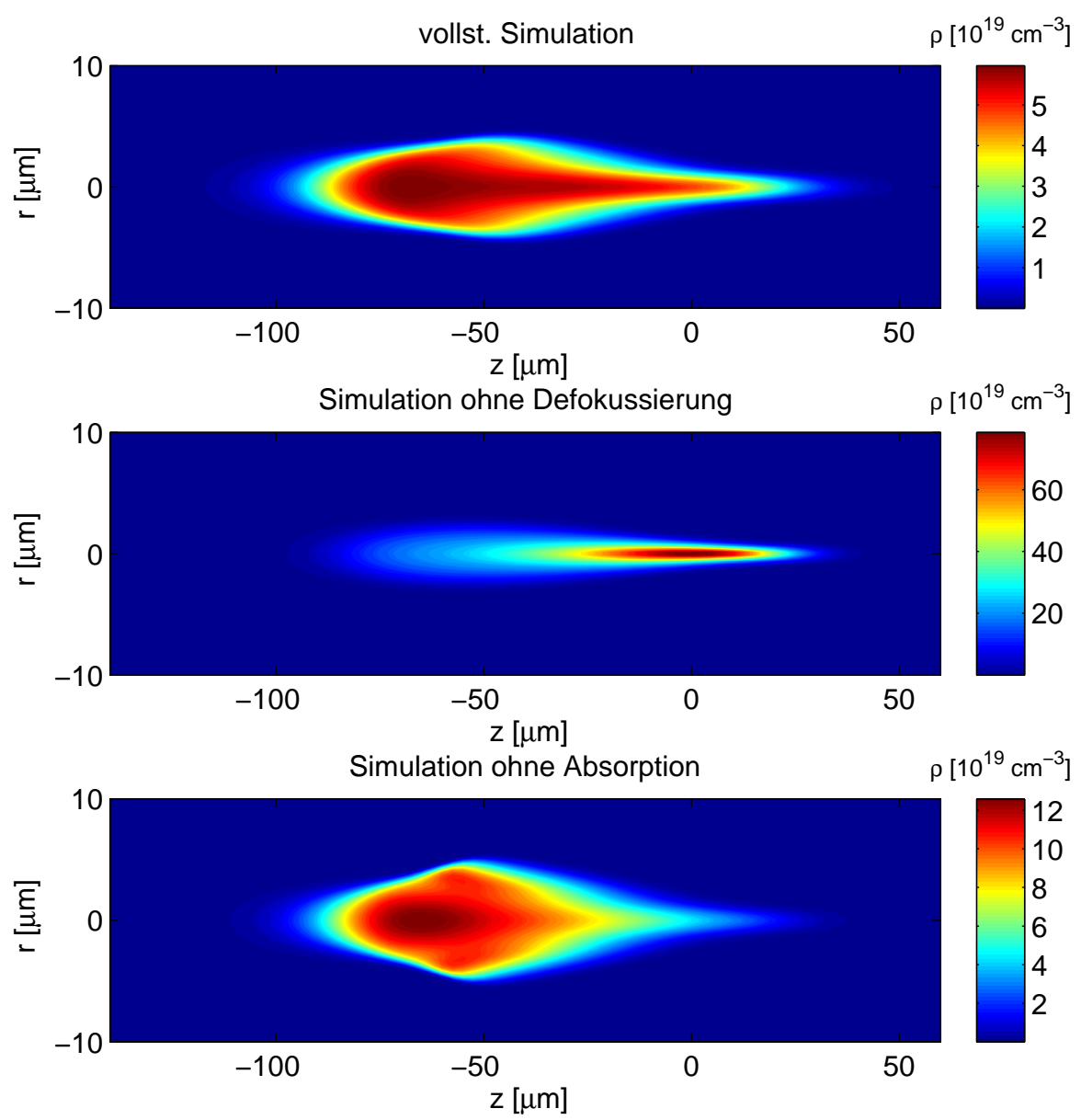

Abbildung 5.4: Vergleich des entstehenden Plasmas bei Vernachlässigung wahlweise der für die Defokussierung und der für die Absorption verantwortlichen Terme, $\mathrm{E}=0.5 \mu \mathrm{J}$.

Vergleicht man die vorgestellten Ergebnisse mit Berechnungen von Arnold et al. 4, bei denen ähnliche Parameter verwendet wurden, so ergibt sich eine gute Übereinstimmung der Elektronendichte und Pulsformen. Dies kann als Bestätigung für die korrekte Funktion der entwickelten Simulation aufgefasst werden.

\subsection{Vergleich mit experimentellen Daten}

Im Folgenden sollen die berechneten Plasmen mit experimentellen Ergebnissen (vgl. Abschnitt 5.3) verglichen werden. Dazu dienen Aufnahmen von Kavitationsblasen, die kurz nach dem Durchlaufen des Pulses aufgenommen wurden. Als Vergleichsgröße wird die Länge der Blase entlang der optischen Achse betrachtet. Diese Länge eignet sich deshalb für einen Vergleich, weil sie sich beim Aufschwingen einer (elongierten) Blase vergleichsweise wenig ändert (siehe Ab- 


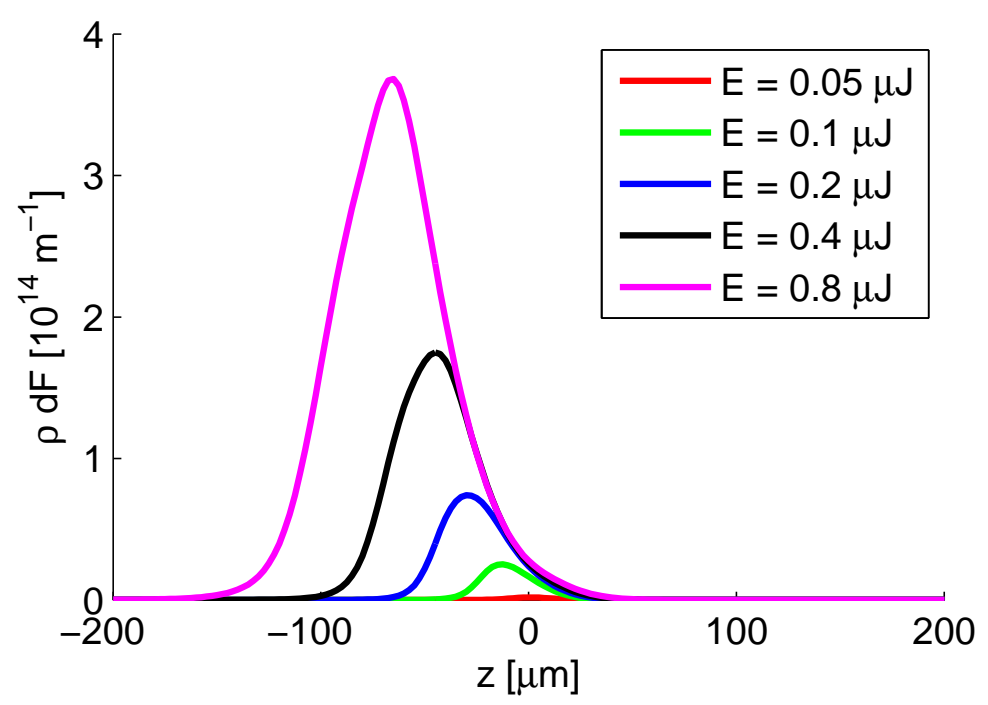

Abbildung 5.5: Elektronendichte (integriert) in Abhängigkeit von $z$ für unterschiedliche Pulsenergien (vgl. Abbildung 5.2).

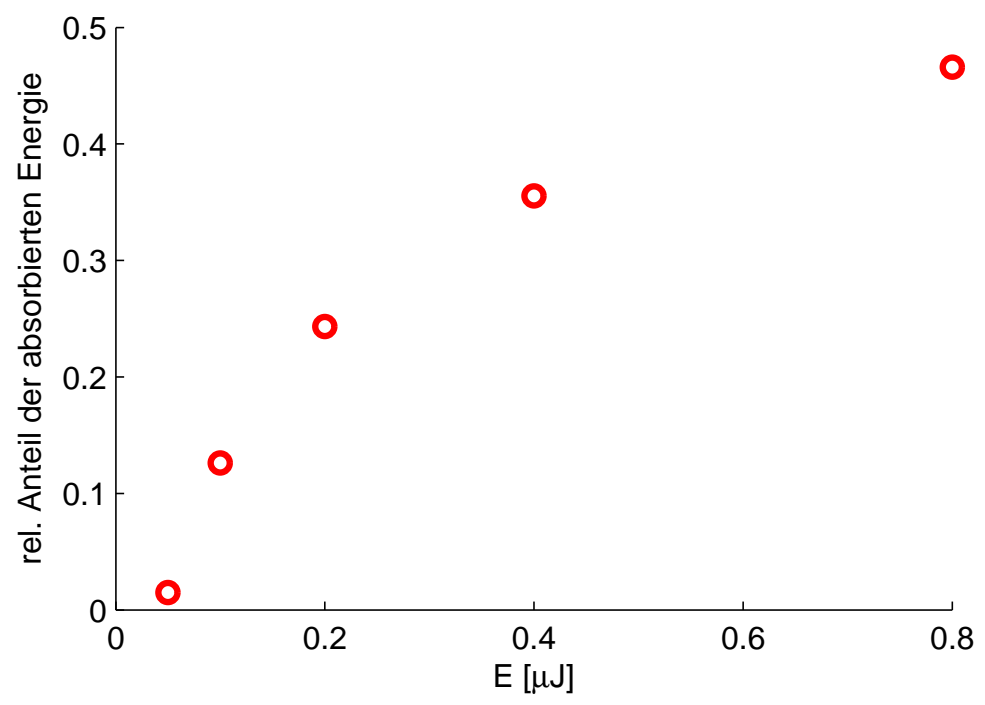

Abbildung 5.6: Verhältnis der absorbierten Energie zur Gesamtenergie in Abhängigkeit der Pulsenergie (vgl. Abbildung 5.2).

bildung [5.7, [33]). Damit kann angenommen werden, dass die Länge der Blase (in Richtung der optischen Achse) der des Plasmas in guter Näherung entspricht.

Abbildung 5.8 zeigt einen Vergleich der Ausdehnung der Blasen im Experiment mit der Ausdehnung der berechneten Plasmen. Dabei wurde jeweils die Länge der Strecke vom zum Systemsystem zeigenden Ende der Blase (bzw. des Plasmas) bis zum linearen Fokus gemessen. Im Fall des Plasmas wurden zur Längebestimmung die Durchbruchschwellen $\rho_{\text {thr }}=10^{18} \mathrm{~cm}^{-3}$ und $\rho_{\text {thr }}=10^{19} \mathrm{~cm}^{-3}$ gewählt. Der erste Wert entspricht dabei dem von Kennedy 45] definierten 


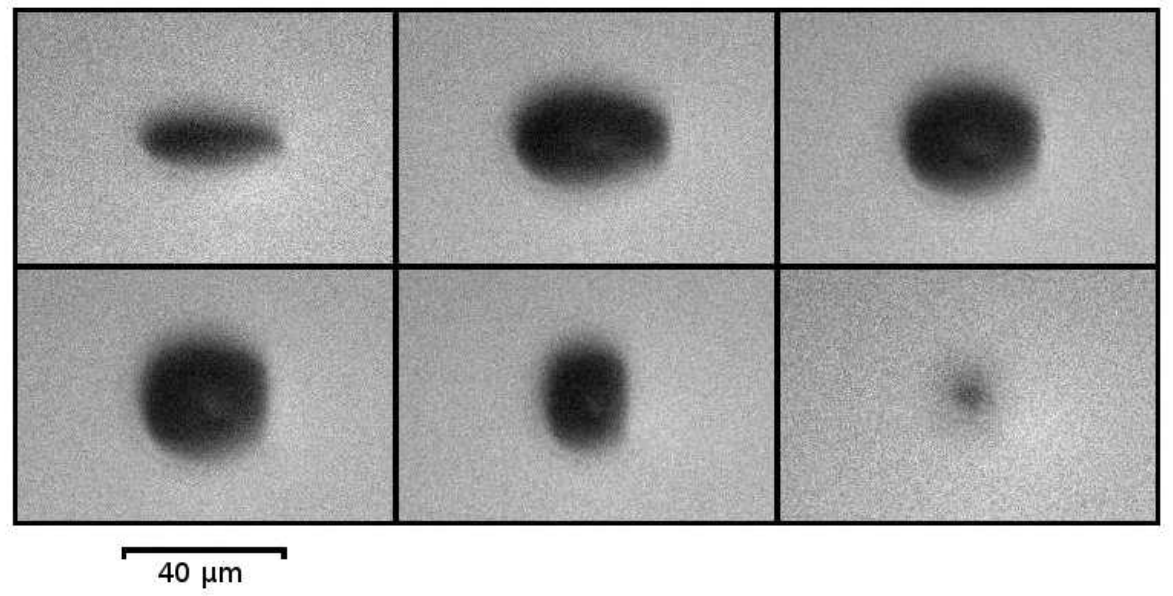

Abbildung 5.7: Aufschwingen und Kollaps einer fs-Kavitationsblase $(\mathrm{E}=0.26 \mu \mathrm{J})$, Aufnahme des ersten Bildes $200 \mathrm{~ns}$ nach Durchlaufen des Pulses, Bildabstand 500 ns bei einer Belichtungszeit von 200 ns, Aufnahmen von Kröninger [49].

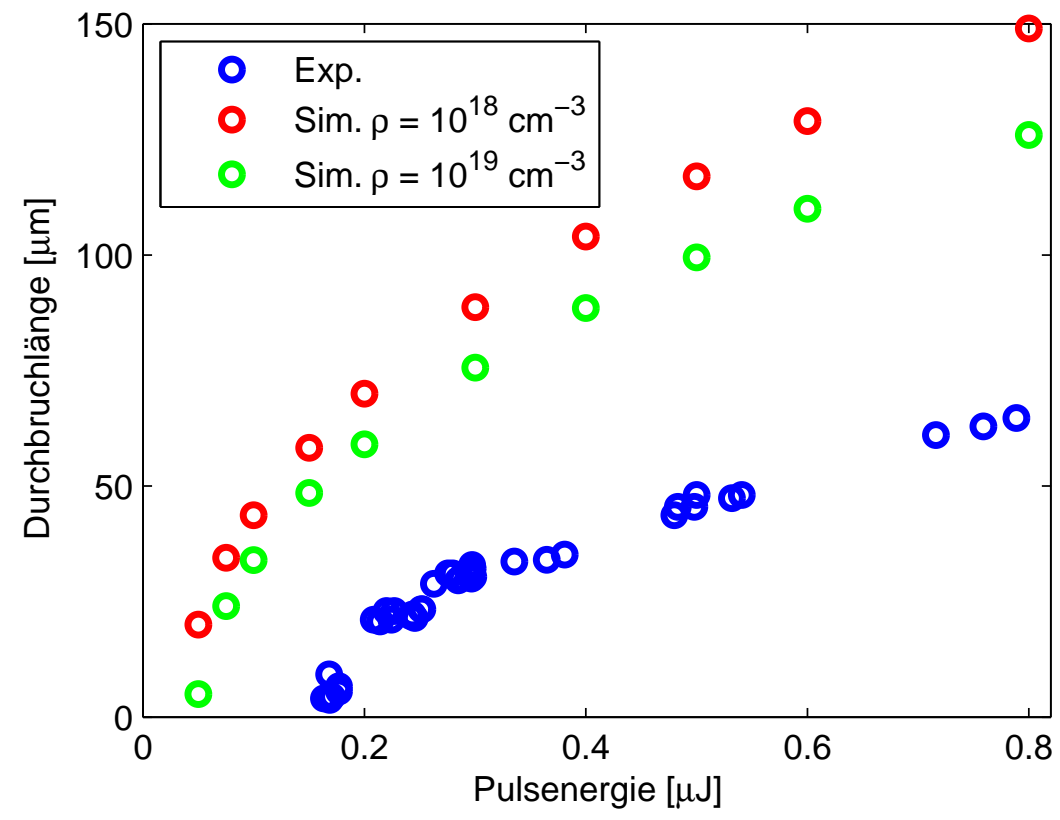

Abbildung 5.8: Vergleich des Abstandes vom Beginn des berechneten Plasmas (bzw. der beobachteten Blase) bis zum linearen Fokus für verschiedene Pulsenergien.

„bubble endpoint", der zweite wurde (etwas willkürlich) in der Größenordnung der maximalen Elektronendichte (vgl. Abbildung 5.2) gewählt. Die anderen in Abschnitt 4.6 diskutierten Durchbruchsschwellen liegen über den berechneten Maximaldichten und scheinen für fs-Pulse zu hoch angesetzt zu sein.

Man erkennt bei der Betrachtung von Abbildung [5.8, dass die berechneten 
Plasmen deutlich stärker entlang der optischen Achse ausgedehnt sind als die im Experiment beobachteten Blasen. Allerdings fällt auch auf, dass die Abhängigkeit der betrachteten Längen von der Pulsenergie für die berechneten und experimentellen Daten ähnlich ist. Dies bestätigt sich, wenn die Energie für die berechneten Größen geeignet skaliert wird. In Abbildung 5.9 ist der Vergleich (für die Durchbruchsschwelle $\rho_{\mathrm{thr}}=10^{19} \mathrm{~cm}^{-3}$ ) dargestellt, wenn die Energie um den Faktor 3.5 geändert wird.

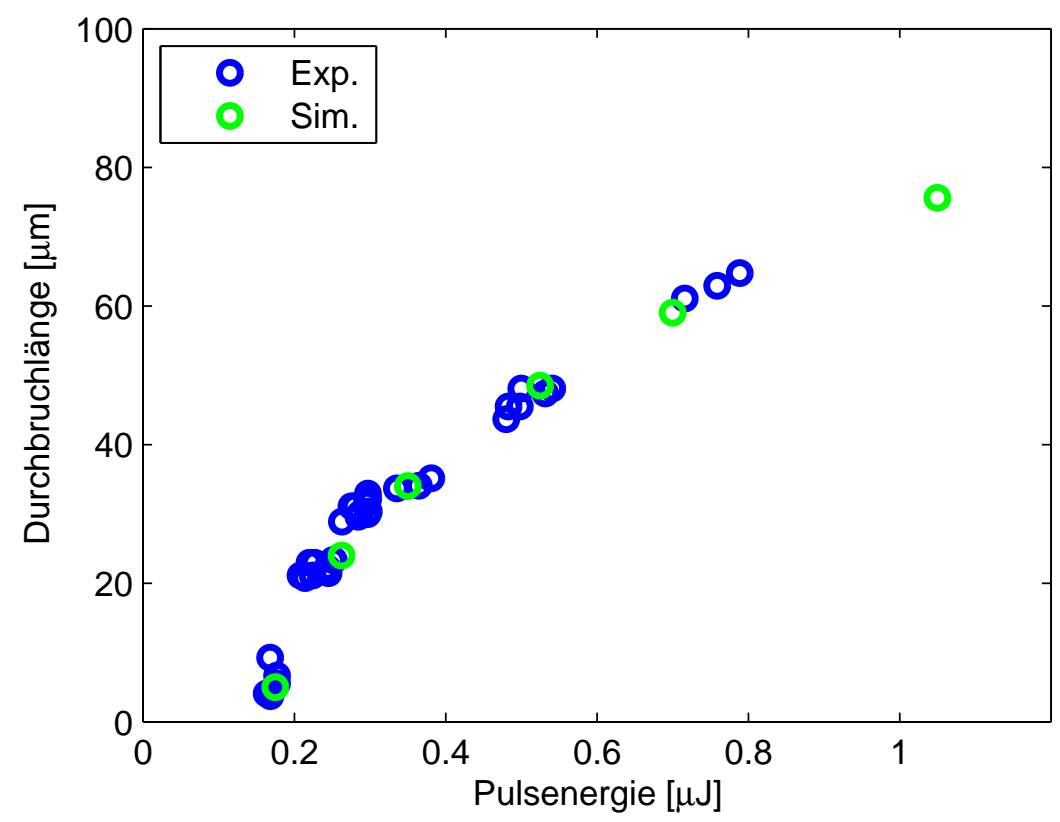

Abbildung 5.9: Vergleich der Plasma- bzw. Blasenlänge bei Skalierung der Energie mit dem Faktor 3.5, Durchbruchsschwelle $\rho_{\text {thr }}=10^{19} \mathrm{~cm}^{-3}$.

Abbildung 5.10 zeigt einen Vergleich zwischen den aufgenommenen Blasen und der Elektronendichte unter Verwendung der Skalierung der Energie. Man erkennt, dass - wie nach Abbildung 5.9 zu erwarten ist - die Längen gut übereinstimmen, die Ausdehnung der Plasmen senkrecht zur Ausbreitungsrichtung aber deutlich geringer als bei den Blasen ist. Dies muss allerdings kein Widerspruch sein, da die Blasenaufnahmen das Aufschwingen der Blase über 200 ns wiedergeben.

Es zeigt sich also, dass mit der Anpassung der Energie (und der geeigneten Wahl einer Durchbruchsschwelle) eine gute Übereinstimmung zwischen Simulation und Experiment hergestellt werden kann.

Ohne diese Anpassung ergeben sich in der Simulation wie in Abbildung 5.8 zu sehen deutlich zu große Plasmen. Dies kann unterschiedliche Ursachen haben.

So ist es möglich, dass im Experiment die Energie des Pulses (vor Erreichen des Fokus) überschätzt wurde, wenn die Strahlungsverluste z. B. durch Streuver- 

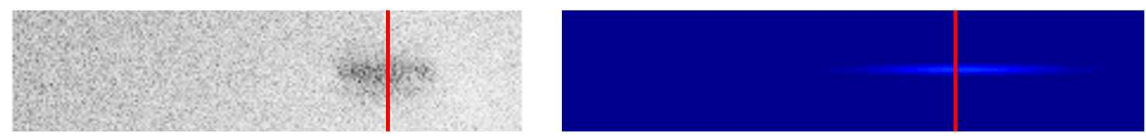

$E=0.16 \mu$
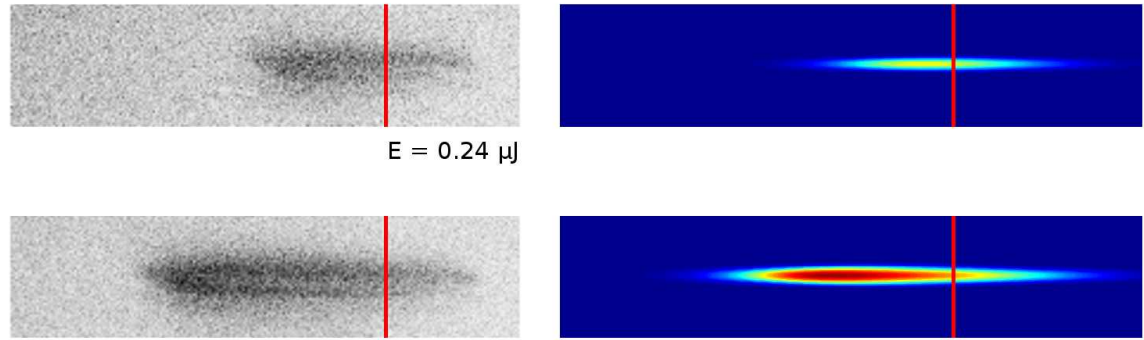

$E=0.39 \mu$
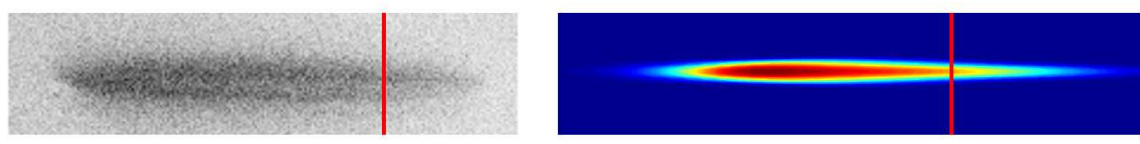

$E=0.52 \mu$

$20 \mu \mathrm{m}$

Abbildung 5.10: Vergleich der experimentellen Aufnahmen mit Ergebnissen der Simulation. Dabei wurde die Energie der simulierten Werte angepasst (s. Abbildung 5.9). Die rote Linie gibt die Lage des linearen Fokus an. Die Aufnahme der Blasen geschah 100 ns nach Durchlauf des Pulses bei einer Belichtungszeit von 200 ns. Die Farbskala der Elektronendichten entspricht der aus Abbildung 5.2.

luste an den Linsen höher als angenommen sind, so dass die Energieanpassung gerechtfertigt ist.

Ferner ist das tatsächliche Strahlprofil (und die Pulsform) des Lasers (insbesondere nach Durchlaufen des Linsensystems [7]) nicht bekannt und eine mögliche Fehlerquelle, wie das Beispiel für einen Puls mit einem nichtgaußschen räumlichen Strahlprofil im nächsten Abschnitt zeigt.

\subsection{Einfluss des Strahlprofils auf die Plasmabil- dung}

In diesem Abschnitt wird die Berechnung der Elektronendichte für einen Puls vorgestellt, der räumlich ein nicht-Gaußsches Strahlprofil besitzt. Dafür wird als Beispiel ein Puls ausgewählt, dessen elektrisches Feld in $r$-Richtung durch eine Linearkombination der ersten beiden Gauß-Laguerre-Moden (vgl. Abschnitt 3.1.2 beschrieben werden kann. Die Berechnung des Strahlprofils vor Durchlaufen des Fokus erfolgt mit Hilfe des Kirchhoffschen Beugungsintegrals 
in Fresnelscher Näherung in Zylinderkoordinaten (vgl. Abschnitt 3.1.5. Das Verhältnis der Gauß-Laguerre-Moden wurde durch Vergleich des Verlaufs des Strahlradius mit den theoretischen Kurven aus Abbildung[3.3] so gewählt, dass für die Strahlqualität $M^{2}=1.5$ gilt.
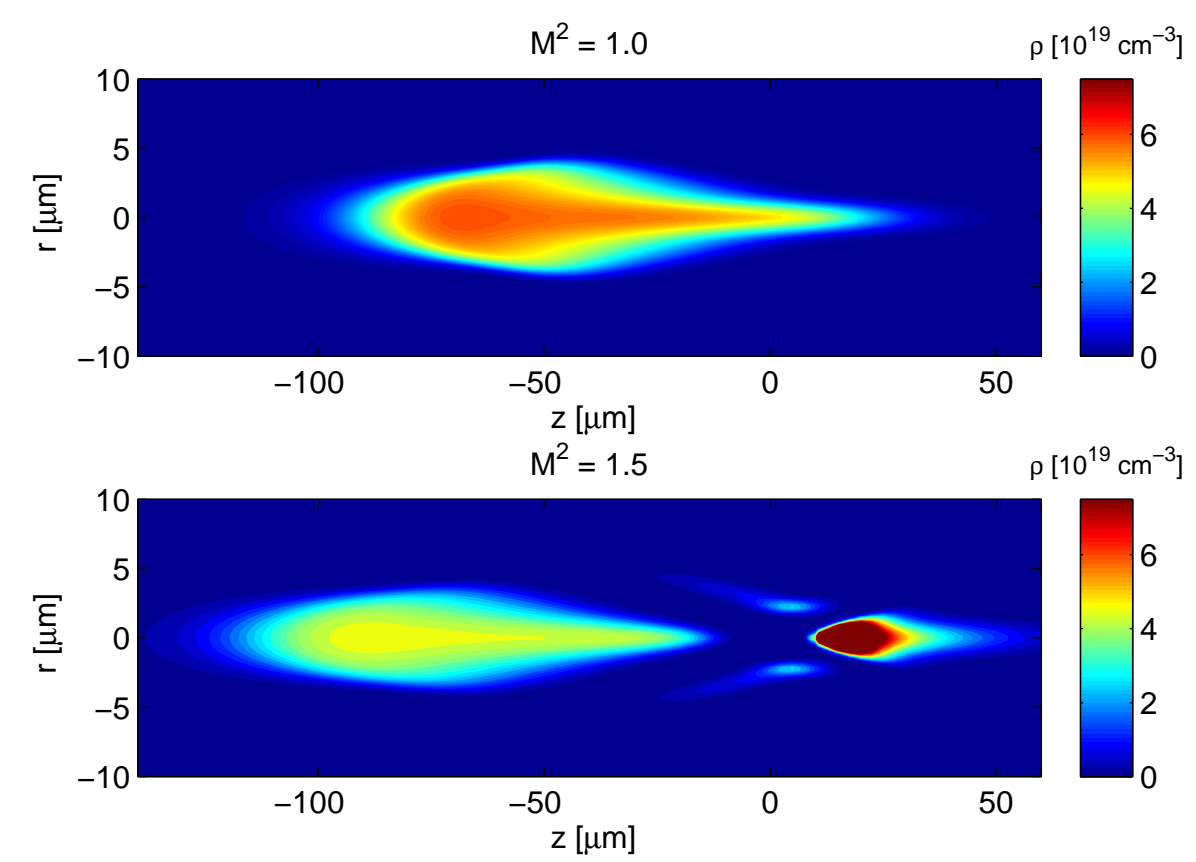

Abbildung 5.11: Beispiel für ein durch einen Puls mit Beugungsmaßzahl $M^{2}=1.5$ erzeugtes Plasma im Vergleich zu einem Plasma, das durch einen Puls mit Gaußschem Strahlprofil generiert wurde. Für eine bessere Vergleichbarkeit wurden identische Skalierungen verwendet, wodurch im unteren Bild die auftretenden hohen Elektronendichten nicht mehr aufgelöst werden können.

Abbildung 5.11 zeigt das resultierende Plasma. Der Vergleich mit dem durch einen Puls mit Gaußschem Strahlprofil generierten Plasma zeigt deutliche Unterschiede. So bildet sich für den nicht-Gaußschen Puls schon für kleineres $z$ ein Plasma aus. Hinter diesem ist zudem ein (kleinerer) Bereich zu erkennen, in dem die Elektronendichte ein Vielfaches der in den anderen Beispiel erreichten Dichten erreicht. Vermutlich werden an dieser Stelle die weiter von der optischen Achse entfernten Pulsanteile fokussiert.

Diese Beispiel zeigt, dass selbst für ein relativ kleines $M^{2}$ das Strahlprofil die Plasmabildung deutlich beeinflussen kann. Es muss aber beachtet werden, dass das vorgestellte Beispiel nur die Auswirkung eines Strahlprofils auf die Plasmaentstehung wiedergibt und nicht verallgemeinert werden kann. 


\section{Kapitel 6}

\section{Zusammenfassung und Ausblick}

\subsection{Zusammenfassung}

In der vorliegenden Arbeit wurde der optische Durchbruch von fs-Laserpulsen in Wasser numerisch untersucht.

Dazu wurde zunächst die Ausbreitung von Laserpulsen in nichtlinearen Medien unter Vernachlässigung der Ionisation betrachtet und die zur Berechnung der Propagation verwendete Ausbreitungsgleichung hergeleitet. Die zur Lösung dieser Gleichung verwendeten Verfahren wurden vorgestellt. Anhand von Beispielen zur Ausbreitung nicht räumlich ausgedehnter few-cycle-Pulse konnten die Rechnungen durch einen Vergleich mit durch die FDTD-Methode berechneten Ergebnissen verifiziert werden.

Das verwendete Verfahren wurde im Folgenden um die Berechnung von (räumlich) ausgedehnten Pulsen erweitert. Dazu wurde insbesondere die Fokussierung von Strahlung mit Gaußschem Profil betrachtet. Desweiteren wurde diskutiert, wie sich Abweichungen von diesem Profil auf die Fokussierbarkeit auswirken. Ein weiterer Schwerpunkt lag auf der Diskussion des bei der Fokussierung von fs-Pulsen wichtigen Effektes der Selbstfokussierung.

Im weiteren Verlauf wurde vorgestellt, wie das im Fokus entstehende Plasma beschrieben werden kann. Die Beschreibung des Plasmas erfolgte dabei nach dem Drude-Modell, wobei für die Photoionisation die Keldysh-Theorie verwendet wurde. Die zeitliche Entwicklung der (maximalen) Elektronendichte eines durch einen fs-Laserpuls generierten Plasmas wurde (unter Vernachlässigung der Pulsausbreitung) berechnet und mit der Plasmaentstehung durch ns-Laserpulse verglichen.

Anschließend wurde diskutiert, wie die Berechnungen zur Pulsausbreitung und zur Plasmaentstehung kombiniert werden können. Mit diesen Rechnungen erhaltene Ergebnisse für das entstehende Plasma und die Veränderung des Pulses beim Durchlaufen des Fokus wurden vorgestellt. Die berechneten Plasmen und 
Pulsformen zeigen eine gute Übereinstimmung mit Ergebnissen von Arnold et al. [4], bei denen ähnliche Parameter verwendet wurden.

Weiterhin wurde ein Vergleich mit experimentellen Daten durchgeführt. Dazu wurden Aufnahmen von Kavitationsblasen verwendet [49, die durch optische Kavitation mit fs-Laserpulsen erzeugt wurden. Dabei zeigte sich, dass die berechneten Plasmen im Vergleich zu den beobachteten Blasen stärker entlang der optischen Achse ausgedehnt sind. Durch eine Korrektur der Energie konnte jedoch eine gute Übereinstimmung zwischen den Ergebnissen der Simulation und des Experiments erreicht werden.

\subsection{Ausblick}

Es sind verschiedene Veränderungen und Erweiterungen der vorgestellten Simulation wie auch beim Vergleich mit dem Experiment denkbar.

Bei den vorgestellten Berechnungen wurde für das Plasma und insbesondere für die Kaskadenionisation ein recht einfaches Modell verwendet. Dies kann erweitert werden, so dass insbesondere auch die Energieverteilung der Elektronen im Plasma berücksichtigt wird [63, 5].

Ferner wäre es möglich, die Untersuchungen auf die Abläufe nach der Plasmabildung auszuweiten, indem insbesondere die thermodynamischen Eigenschaften des Wasser berücksichtigt werden [80]. Damit könnten Anfangswerte für eine Simulation der Dynamik der entstehenden Kavitationsblase [50, 47] erhalten werden, deren Ergebnisse einen direkten Vergleich mit den experimentellen Beobachtungen ermöglichen würden. Um die Berechnungen noch besser mit dem Experiment vergleichen zu können, ist außerdem eine genauere Charakterisierung des Laserpulses (im Experiment) nötig.

Einen weiteren interessanten Untersuchungsaspekt stellen die Möglichkeiten der Beeinflussung des entstehenden Plasmas durch Veränderung der (raumzeitlichen) Pulsform dar. Insbesondere wäre die Bildung eines möglichst runden Plasmas für die Untersuchung von Kavitationsblasen interessant. Dies ist zwar mit (Immersions-) Objektiven mit sehr großer numerischer Apertur annähernd möglich [3], die in der Regel sehr kleine Brennweite verhindert aber ein freies Aufschwingen der Blase.

Diese Arbeit und das zugrunde liegende Simulationsprogramm können also für weitere interessante Untersuchungen zum optischen Durchbruch in Wasser verwendet werden. 


\section{Anhang A}

\section{Symbolverzeichnis}

\section{A.1 Konstanten}

\begin{tabular}{|l|l|l} 
Symbol & Größe & Beschreibung \\
\hline$c$ & $299792458 \mathrm{~m} / \mathrm{s}$ & Vakuumlichtgeschwindigkeit \\
$e$ & $\approx 1.602 \cdot 10^{-19} \mathrm{As}$ & Elementarladung \\
$\epsilon_{0}=1 /\left(c^{2} \mu_{0}\right)$ & $\approx 8.854 \cdot 10^{-12} \mathrm{As} / \mathrm{Vm}$ & elektrische Feldkonstante \\
$m_{e}$ & $\approx 9.109 \cdot 10^{-31} \mathrm{~kg}$ & Masse des Elektrons \\
$\mu_{0}$ & $4 \pi \cdot 10^{-7} \mathrm{Vs} / \mathrm{Am}$ & magnetische Feldkonstante
\end{tabular}

\section{A.2 Variable und sonstige Bezeichnungen}

\begin{tabular}{|l|l|l} 
Symbol & Einheit & Beschreibung \\
\hline$A$ & $\mathrm{~V} / \mathrm{m}$ & komplexe Einhüllende des elektrischen Feldes \\
$A^{\star}$ & 1 & normierte komplexe Einhüllende des elektr. Feldes \\
$A_{\mathrm{c}}$ & $\mathrm{V} / \mathrm{m}$ & Lösung für $A$ mit geringerer Genauigkeit (coarse) \\
$A_{\mathrm{f}}$ & $\mathrm{V} / \mathrm{m}$ & Lösung für $A$ mit höherer Genauigkeit (fine) \\
$A_{\mathrm{g}}$ & $\mathrm{V} / \mathrm{m}$ & Amplitude des elektr. Feldes für Gauß-Strahlen \\
$A_{p m}$ & 1 & Gauß-Laguerre-Funktion \\
$\alpha_{n}$ & $\mathrm{~s}^{n} / \mathrm{m}$ & Absorptionsterm $n$-ter Ordnung \\
$b_{\mathrm{f}}$ & $\mathrm{m}$ & Brennweite des Gauß-Strahls \\
$B$ & $\mathrm{C} / \mathrm{m}^{2}$ & Einhüllende der nichtlinearen Polarisation \\
$\mathbf{B}$ & $\mathrm{Vs} / \mathrm{m}^{2}$ & magnetische Flussdichte \\
$\beta_{0}$ & $1 / \mathrm{m}$ & Realteil der Wellenzahl für $\omega_{0}$ \\
$\beta_{n}$ & $\mathrm{~s}^{n} / \mathrm{m}$ & Dispersionsterm $n$-ter Ordnung \\
$D$ & $\mathrm{~m} 2 / \mathrm{s}$ & Diffusionskonstante \\
$\hat{D}$ & $1 / \mathrm{m}^{2}$ & Dispersionsoperator \\
$\mathbf{D}$ & $\mathrm{C} / \mathrm{m}^{2}$ & elektrische Flussdichte \\
$\partial_{a}$ & $1 /[\mathrm{a}]$ & partielle Ableitung bzgl. $a$
\end{tabular}




\begin{tabular}{|c|c|c|}
\hline Symbol & Einheit & Beschreibung \\
\hline$\triangle$ & $1 / \mathrm{m}^{2}$ & Laplace-Operator \\
\hline$\triangle_{\perp}$ & $1 / \mathrm{m}^{2}$ & Laplace-Operator bzgl. $\mathbf{r}_{\perp}$ \\
\hline $\mathbf{E}$ & $\mathrm{V} / \mathrm{m}$ & elektrisches Feld \\
\hline$\tilde{\mathbf{E}}=\mathcal{F}(\mathbf{E})$ & $\mathrm{V} / \mathrm{m}$ & (zeitliche) Fouriertransf. des elektrischen Feldes \\
\hline$\hat{\mathbf{E}}$ & $\mathrm{V} / \mathrm{m}$ & komplexe Darstellung des elektrischen Feldes \\
\hline$E_{0}$ & $\mathrm{~V} / \mathrm{m}$ & Amplitude des elektrischen Feldes \\
\hline$E^{\text {Kollaps }}$ & $\mathrm{V} / \mathrm{m}$ & Strahlprofil im Kollaps \\
\hline $\mathrm{E}$ & 1 & vollst. elliptisches Integral 2. Art \\
\hline E & VAs & Energie \\
\hline $\mathrm{E}_{\mathrm{avg}}$ & VAs & durchschnittliche Energie der Elektronen \\
\hline $\mathrm{E}_{\mathrm{b}}$ & VAs & Bandlücke \\
\hline$E_{\text {eff }}$ & VAs & effektive Bandlücke \\
\hline$E_{\text {ion }}$ & VAs & Energie zur Erzeugung eines freien Elektrons \\
\hline $\mathrm{E}_{\mathrm{st}}$ & VAs & Energie für Stoßionisation \\
\hline$\epsilon$ & 1 & Permittivität oder rel. Dielektrizitätskonstante \\
\hline$\eta_{\mathrm{ph}}$ & $1 / \mathrm{m}^{3} \mathrm{~s}$ & Rate für Photoionisation \\
\hline$\eta_{\mathrm{st}}$ & $1 / \mathrm{s}$ & Rate für Stoßionisation \\
\hline$\eta_{\text {diff }}$ & $1 / \mathrm{s}$ & Rate für Diffusion \\
\hline$\eta_{\text {rec }}$ & $\mathrm{m}^{3} / \mathrm{s}$ & Rekombinationsrate \\
\hline$f$ & $\mathrm{~m}$ & Brennweite \\
\hline$F$ & $m^{2}$ & Flächenelement \\
\hline GVD & $\mathrm{s}^{2} / \mathrm{m}$ & Gruppengeschwindigkeitsdispersion \\
\hline$\gamma$ & 1 & Keldysh-Parameter \\
\hline$\Gamma$ & 1 & Funktion des Keldysh-Parameters \\
\hline$h$ & $\mathrm{~m}$ & Schrittweite bezüglich $z$ \\
\hline $\mathbf{H}$ & $\mathrm{A} / \mathrm{m}$ & magnetische Feldstärke \\
\hline$I$ & $\mathrm{VA} / \mathrm{m}^{2}$ & Intensität \\
\hline j & $\mathrm{A} / \mathrm{m}^{2}$ & Stromdichte \\
\hline$J_{0}$ & 1 & Besselfunktion 0. Ordnung 1. Gattung \\
\hline$\underset{\sim}{k}$ & $1 / \mathrm{m}$ & Wellenzahl \\
\hline$\tilde{k}$ & $1 / \mathrm{m}$ & Fouriertransformierte der Wellenzahl \\
\hline$k_{0}$ & $1 / \mathrm{m}$ & Wellenzahl im Vakuum \\
\hline$k_{\perp}$ & $\mathrm{m}^{2} / \mathrm{s}$ & Faktor in Diffusionsgleichung \\
\hline k & 1 & Photonenanzahl \\
\hline$K$ & 1 & Strahlqualität \\
\hline K & 1 & vollst. elliptisches Integral 1. Art \\
\hline$l$ & $\mathrm{~m}$ & Breite des Kollapsprofils \\
\hline$L_{\text {diff }}$ & $\mathrm{m}$ & Diffusionslänge \\
\hline$L_{p}$ & 1 & Laguerre-Polynom der Ordnung $p$ \\
\hline$\hat{L}$ & $1 / \mathrm{m}$ & Operator (der linearen Anteile) \\
\hline$\lambda$ & $\mathrm{m}$ & Wellenlänge \\
\hline$\lambda_{0}$ & $\mathrm{~m}$ & Wellenlänge im Vakuum \\
\hline$n$ & 1 & Brechungsindex \\
\hline$n_{0}$ & 1 & Brechungsindex bzgl. $\omega_{0}$ \\
\hline
\end{tabular}




\begin{tabular}{|c|c|c|}
\hline Symbol & Einheit & Beschreibung \\
\hline$n_{2}$ & $\mathrm{~m}^{2} / \mathrm{VA}$ & nichtlinearer Brechungsindex(-koeffizient) \\
\hline$n_{\text {ges }}$ & 1 & Gesamtbrechungsindex \\
\hline$n_{\mathrm{st}}$ & 1 & Anteil der Stoßionisation am Brechungsindex \\
\hline$N_{\mathrm{cr}}^{\mathrm{Gau} ß}$ & 1 & Faktor für kritische Leistung des Gauß-Profils \\
\hline$N_{\mathrm{cr}}^{\text {Tow }}$ & 1 & Faktor für kritische Leistung des Townes-Profils \\
\hline$\hat{N}$ & $1 / \mathrm{m}$ & Operator (der nichtlinearen Anteile) \\
\hline$m_{e}$ & $\mathrm{~kg}$ & Masse eines Elektrons \\
\hline$m_{h}$ & $\mathrm{~kg}$ & Masse eines Loches \\
\hline$m^{\prime}$ & $\mathrm{kg}$ & reduzierte Masse \\
\hline$m^{\star}$ & $\mathrm{kg}$ & effektive Masse \\
\hline$M^{2}$ & 1 & Beugungsmaßzahl \\
\hline$\mu$ & 1 & Quotient aus Elektron- und Lochmasse \\
\hline$q$ & $\mathrm{~m} / \mathrm{V}^{2}$ & Koeffizient für Stärke der Nichtlinearität \\
\hline $\mathbf{P}$ & $\mathrm{C} / \mathrm{m}^{2}$ & Polarisation \\
\hline $\mathbf{P}_{1}$ & $\mathrm{C} / \mathrm{m}^{2}$ & Polarisation (linearer Anteil) \\
\hline $\mathbf{P}_{\mathrm{nl}}$ & $\mathrm{C} / \mathrm{m}^{2}$ & Polarisation (nichtlinearer Anteil) \\
\hline $\mathbf{P}_{\mathrm{ph}}$ & $\mathrm{C} / \mathrm{m}^{2}$ & Polarisation durch Photonenionisation \\
\hline $\mathbf{P}_{\mathrm{st}}$ & $\mathrm{C} / \mathrm{m}^{2}$ & Polarisation durch Stoßionisation \\
\hline$P$ & VA & Leistung \\
\hline$P_{\text {gauss }}$ & VA & Leistung des gaußförmigen Strahlprofils \\
\hline$P_{\mathrm{cr}}^{\mathrm{Gau}}$ & VA & kritische Leistung des Gauß-Profils \\
\hline $\mathrm{P}_{\mathrm{cr}}^{\mathrm{T} o w}$ & VA & kritische Leistung des Townes-Profils \\
\hline$\phi$ & 1 & Zylinderkoordinate \\
\hline$\phi_{\mathrm{NL}}$ & 1 & maximale Phasendrehung \\
\hline$\psi$ & 1 & Phase \\
\hline$\Phi$ & 1 & Dawson-Integral \\
\hline$r$ & $\mathrm{~m}$ & Zylinderkoordinate \\
\hline $\mathbf{r}$ & $\mathrm{m}$ & Ort \\
\hline $\mathbf{r}_{\perp}$ & $\mathrm{m}$ & Anteil von $\mathbf{r}$ senkrecht $\mathrm{zu} z$ \\
\hline$R$ & $\mathrm{~m}$ & Krümmungsradius der Phasenfront \\
\hline$R_{12}$ & 1 & Reflexionsvermögen \\
\hline$\rho$ & $1 / \mathrm{m}^{3}$ & Elektronenzahldichte (Leitungsband) \\
\hline$\rho_{\mathrm{cr}}$ & $1 / \mathrm{m}^{3}$ & kritische Elektronenzahldichte \\
\hline$\rho_{\mathrm{V}}$ & $1 / \mathrm{m}^{3}$ & Elektronenzahldichte im Valenzband \\
\hline$\rho_{\text {ges }}$ & $1 / \mathrm{m}^{3}$ & gesamte Elektronenzahldichte \\
\hline$\rho_{\mathrm{thr}}$ & $1 / \mathrm{m}^{3}$ & Elektronenzahldichte für optischen Durchbruch \\
\hline$\rho^{\prime}$ & $\mathrm{C} / \mathrm{m}^{3}$ & Raumladungsdichte (Maxwell-Gleichung) \\
\hline$s$ & $\mathrm{~m}^{2}$ & Koeffizient beim Crank-Nicolson-Verfahren \\
\hline$\sigma$ & $\mathrm{m}^{2}$ & Wirkungsquerschnitt inverse Bremsstrahlung \\
\hline$t$ & $\mathrm{~s}$ & Zeit \\
\hline$t_{0}$ & $\mathrm{~S}$ & Pulslänge nach Ansatz \\
\hline$t_{\mathrm{FWHM}}$ & s & Pulslänge bzgl. FWHM der Intensität \\
\hline$\tau$ & $\mathrm{s}$ & Zeit im mitbewegten Koordinatensystem \\
\hline$\tau_{\text {st }}$ & $\mathrm{S}$ & Relaxationszeit für Elektron-Phonon-Streuung \\
\hline
\end{tabular}




\begin{tabular}{|l|l|l} 
Symbol & Einheit & Beschreibung \\
\hline$\theta$ & 1 & (halber) Divergenzwinkel \\
$v$ & $\mathrm{~m} / \mathrm{s}$ & Phasengeschwindigkeit \\
$v_{g}$ & $\mathrm{~m} / \mathrm{s}$ & Gruppengeschwindigkeit \\
$w$ & $\mathrm{~m}$ & Strahlradius \\
$w_{\mathrm{f}}$ & $\mathrm{m}$ & Strahltaille \\
$w_{0}$ & $\mathrm{~m}$ & Strahlradius nach Ansatz \\
$\omega$ & $1 / \mathrm{s}$ & Kreisfrequenz \\
$\omega_{0}$ & $1 / \mathrm{s}$ & mittlere Kreisfrequenz des Laserpulses \\
$\omega_{\mathrm{p}}$ & $1 / \mathrm{s}$ & Plasmafrequenz \\
$\chi_{(n)}$ & 1 & elektrische Suszeptibilität \\
$\chi^{(n)}$ & $(\mathrm{V} / \mathrm{m})^{(1-n)}$ & Suszeptibilität n-ter Ordnung \\
$\chi_{\mathrm{ges}}$ & 1 & (effektive) Suszeptibilität \\
$\chi_{\mathrm{l}}$ & 1 & linearer Anteil der Suszeptibilität \\
$\chi_{\mathrm{nl}}$ & 1 & nichtlinearer Anteil der Suszeptibilität \\
$\chi_{\mathrm{ph}}$ & 1 & Suszeptibilität durch Photonenionisation \\
$\chi_{\mathrm{st}}$ & 1 & Suszeptibilität durch Stoßionisation \\
$x$ & $\mathrm{~m}$ & Koordinate in transversaler Richtung \\
$\xi$ & $\mathrm{m}$ & Koordinate der Ausbreitungsrichtung (mitbewegt) \\
$y$ & $\mathrm{~m}$ & Koordinate in transversaler Richtung \\
$z$ & $\mathrm{~m}$ & Koordinate der Ausbreitungsrichtung \\
$z_{\mathrm{f}}$ & $\mathrm{m}$ & Brennpunkt des fokussierten Strahls \\
$z_{\mathrm{sf}}$ & $\mathrm{m}$ & Brennweite der Selbstfokussierung \\
$z_{\mathrm{f}+\mathrm{sf}}$ & $\mathrm{m}$ & kombinierte Brennweite \\
$z_{\mathrm{R}}$ & $\mathrm{m}$ & Rayleigh-Länge \\
$z_{\mathrm{R}, \mathrm{f}}$ & $\mathrm{m}$ & Rayleigh-Länge des fokussierten Strahls \\
& &
\end{tabular}




\section{Anhang B}

\section{Fouriertransformation und Faltungssatz}

In der vorliegende Arbeit wird die Fouriertransformation und die entsprechende Rücktransformation wie folgt definiert:

$$
\begin{aligned}
\tilde{f}(\omega) & =\mathcal{F}(f(t))=\int_{-\infty}^{\infty} d t e^{-i \omega t} f(t) \\
f(t) & =\mathcal{F}^{-1}(\tilde{f}(\omega))=\frac{1}{2 \pi} \int_{-\infty}^{\infty} d \omega e^{i \omega t} \tilde{f}(\omega)
\end{aligned}
$$

Damit erhält man die folgenden Faltungssätze:

$$
\begin{array}{rlr}
\mathcal{F}(A \cdot B) & =\frac{1}{2 \pi} \mathcal{F}(A) * \mathcal{F}(B) \\
\mathcal{F}(A * B) & = & \mathcal{F}(A) \cdot \mathcal{F}(B)
\end{array}
$$




\section{Literaturverzeichnis}

[1] G. P. Agrawal: Nonlinear Fiber Optics, Second Edition. Academic Press, 1995.

[2] S. A. Akhmanov, A. P. Sukhorukov und R. V. Khokhlov: Self-focusing and diffraction of light in a nonlinear medium. Sov. Phys. Uspekhi 10, 609-636, 1968.

[3] C. L. Arnold: Erzeugung optischer Durchbrüche bei hoher numerischer Apertur: Numerische Simulationen zur Submikrometer-Manipulation transparenter Materialien und biologischer Zellen mit ultrakurzen Pulsen. Dissertation, Hannover, 2007.

[4] C. L. Arnold, A. Heisterkamp, W. Ertmer und H. Lubatschowski: Streak formation as side effect of optical breakdown during processing the bulk of transparent Kerr media with ultra-short laser pulses. Appl. Phys. B 80(2), 247-253, 2005.

[5] C. L. Arnold, A. Heisterkamp, W. Ertmer und H. Lubatschowski: Computational model for nonlinear plasma formation in high NA micromachining of transparent materials and biological cells. Opt. Expr. 15(16), 10303-10317, 2007.

[6] N. Bloembergen: Laser-Induced Electric Breakdown in Solids. J. Quant. Electr. 10(3), 375-386, 1974.

[7] Z. Bor: Distortion of femtosecond laser pulses in lenses. Opt. Lett. 14(2), 119-121, 1989.

[8] M. Born und E. Wolf: Principles of Optics. Cambridge University Press, 1999.

[9] R. W. Boyd, S. G. Lukishova und Y. R. Shen (Eds.): Self-focusing: Past and Present. Springer, 2009.

[10] T. Brabec und F. Krausz: Nonlinear Optical Pulse Propagation in the Single-Cycle Regime. Phys. Rev. Lett. 78(17), 3282-3285, 1997. 
[11] A. Braun, G. Korn, X. Liu, D. Du, J. Squier und G. Mourou: Selfchanneling of high-peak-power femtosecond laser pulses in air. Opt. Lett. 20(1), 73-75, 1995.

[12] A. Brodeur, C. Y. Chien, F. A. Ilkov, S. L. Chin, O. G. Kosareva und V. P. Kandidov: Moving focus in the propagation of ultrashort laser pulses in air. Opt. Lett. 22(5), 304-306, 1997.

[13] I. N. Bronstein, K. A. Semendjajew, G. Musiol und H. Mühlig: Taschenbuch der Mathematik. Harri Deutsch, Frankfurt a. M., 1997.

[14] P. Chernev und V. Petrov: Self-focusing of light pulses in the presence of normal group-velocity dispersion. Opt. Lett. 17(3), 172-174, 1992.

[15] R. Y. Chiao, E. Garmire und C. H. Townes: Self-Trapping of Optical Beams. Phys. Rev. Lett. 13(15), 479-482, 1964.

[16] R. Y. Chiao, E. Garmire und C. H. Townes: Self-Trapping of Optical Beams (Erratum). Phys. Rev. Lett. 14(25), 1056, 1965.

[17] S. L. Chin, W. Liu, O. G. Kosareva und V. P. Kandidov: The Physics of Intense Femtosecond Laser Filamentation. In: R. W. Boyd, S. G. Lukishova, Y. R. Shen (Eds.): Self-focusing: Past and Present S. 349-370, 2009.

[18] A. Couairon und A. Mysyrowitz: Self-focusing and Filamentation of Femtosecond Pulses in Air and Condensed Matter: Simulations and Experiments. In: R. W. Boyd, S. G. Lukishova, Y. R. Shen (Eds.): Self-focusing: Past and Present S. 297-322, 2009.

[19] J. Crank und P. Nicolson: A practical method for numerical evaluation of solutions of partial differential equations of the heat-conduction type. Proc. Camb. Phil. Soc. 43, 50-67, 1947.

[20] E. L. Dawes und J. H. Marburger: Computer Studies in Self-Focusing. Phys. Rev. 179(3), 862-868, 1969.

[21] H. G. Dawson: On the Numerical Value of Formula $\int_{0}^{h} e x^{2} d x$. Proc. London Math. Soc. 1-29(1), 519-522, 1897.

[22] F. Docchio: Lifetimes of Plasmas Induced in Liquids and Ocular Media by Single Nd:YAG Laser Pulses of Different Duration. Europhys. Lett. 6(5), 407-412, 1988.

[23] P. Drude: Zur Elektronentheorie der Metalle. Annalen der Physik 306(3), 566-613, 1900.

[24] J. Eichler, L. Dünkel und B. Eppich: Die Strahlqualität von Lasern - Wie bestimmt man Beugungsmaßzahl und Strahldurchmesser in der Praxis? Laser Technik Journal 2(63-66), 187401, 2004. 
[25] J. Eichler und H. J. Eichler: Laser: Bauformen, Strahlführung, Anwendungen, 6. Auflage. Springer, 2006.

[26] C. H. Fan, J. Sun und J. P. Longtin: Breakdown threshold and localized electron density in water induced by ultrashort laser pulses. J. Appl. Phys. 91(4), 2530-2536, 2002.

[27] Q. Feng, J. V. Moloney, A. C. Newell, E. M. Wright, K. Cook, P. K. Kennedy, B. A. Rockwell und C. R. Thompson: Theory and Simulation on the Threshold of Water Breakdown Induced by Focused Ultrashort Laser Pulses. J. Quant. Electr. 33(2), 127-137, 1997.

[28] G. Fibich: Some Modern Aspects of Self-focusing Theory. In: R. W. Boyd, S. G. Lukishova, Y. R. Shen (Eds.): Self-focusing: Past and Present S. 416-438, 2009.

[29] G. Fibich und A. L. Gaeta: Critical power for self-focusing in bulk media and in hollow waveguides. Opt. Lett. 25(5), 335-337, 2000.

[30] G. Fibich, N. Gavish und X.-P. Wang: New singular solutions of the nonlinear Schrödinger equation. Physica D 211(3-4), 193 - 220, 2005.

[31] G. Fibich und G. C. Papanicolaou: Self-focusing in the perturbed and unperturbed nonlinear Schrodinger equation in critical dimension. SIAM J. Appl. Math. 60, 183-240, 1999.

[32] J. A. Fleck, J. R. Morris und M. D. Feit: Time-Dependent Propagation of High Energy Laser Beams through the Atmosphere. Appl. Phys. A 10(2), 129-160, 1976.

[33] R. Geisler: Untersuchungen zur laserinduzierten Kavitation mit Nanosekunden- und Femtosekundenlasern. Dissertation, Univ. Göttingen, 2004 .

[34] P. M. Goorjian, A. Taflove, R. M. Joseph und S. C. Hagness: Computational Modeling of Femtosecond Optical Solitons from Maxwell's Equations. IEEE J. Quant. Electr. 28(10), 2416-2422, 1992.

[35] T. D. Grow, A. A. Ishaaya, L. T. Vuong, A. L. Gaeta, N. Gavish und G. Fibich: Collapse dynamics of super-Gaussian Beams. Opt. Expr. 14(12), 5468-5475, 2006.

[36] A. Heisterkamp: Einsatz ultrakurzer Laserpulse in der refraktiven Laserchirurgie. Dissertation, Univ. Hannover, 2002.

[37] M. R. Hermann und Jr. J. A. Fleck: Split-operator spectral method for solving the time-dependent Schrödinger equation in spherical coordinates. Phy. Rev. A 38(12), 6000-6012, 1988. 
[38] C. V. Hile und W. L. Kath: Numerical solutions of Maxwell's equations for nonlinear-optical pulse propagation. J. Opt. Soc. Am. B 13(6), 11351145, 1996.

[39] P. P. Ho und R. R. Alfano: Optical Kerr effect in liquids. Phys. Rev. A 20(5), 2170-2187, 1979.

[40] R. M. Joseph und A. Taflove: FDTD Maxwell's Equations Models for Nonlinear Electrodynamics and Optics. IEEE Trans. Ant. Prop. 45(3), 364-374, 1997.

[41] W. Kaiser: Ultrashort Laser Pulses: Generation and Applications. Springer, Berlin, 1993.

[42] L. V. Keldysh: Ionization in the field of a strong electromagnetic wave. Sov. Phys. JETP 20(5), 1307-1314, 1965.

[43] P. L. Kelley: Self-Focusing of Optical Beams. Phys. Rev. Lett. 15(26), 1005-1008, 1965.

[44] P. L. Kelley: Self-Focusing of Optical Beams (Erratum). Phys. Rev. Lett. 16(9), 384-384, 1966.

[45] P. K. Kennedy: A First-Order Model for Computation of Laser-Induced Breakdown Thresholds in Ocular and Aqueous Media: Part 1 - Theory. IEEE J. Quant. Electr. 31(12), 2241-2249, 1995.

[46] P. K. Kennedy, S. A. Boppart, D. X. Hammer, B. A. Rockwell, G. D. Noojin und W. P. Roach: A First-Order Model for Computation of LaserInduced Breakdown Thresholds in Ocular and Aqueous Media: Part 2 Comparison to Experiment. IEEE J. Quant. Electr. 31(12), 2250-2257, 1995.

[47] K. Köhler: Simulationen zur asphärischen Blasendynamik mit der Randintegralmethode. Diplomarbeit, Univ. Göttingen, 2004.

[48] K. Kopitzki: Einführung in die Festkörperphysik. Teubner, 1993.

[49] D. Kröninger: Laserinduzierte Kavitation im Schallfeld. Diplomarbeit, Univ. Göttingen, 2004.

[50] D. Kröninger, K. Köhler, T. Kurz und W. Lauterborn: Particle tracking velocimetry of the flow field around a collapsing cavitation bubble. Experiments in Fluids 48, 395-408, 10.1007/s00348-009-0743-1, 2010.

[51] W. Lauterborn und T. Kurz: Coherent Optics: Fundamentals and Applications. Springer, Berlin, 2002.

[52] W. Lauterborn und T. Kurz: Physics of bubble oscillations. Rep. Prog. Phys. 73, 106501, 2010. 
[53] W. Lauterborn, T. Kurz, R. Mettin und C. D. Ohl: Experimental and theoretical bubble dynamics. Adv. Chem. Phys. 110, 295-380, 1999.

[54] O. Lindau und W. Lauterborn: Laser-produced cavitation-studied with 100 million frames per second. AIP Conference Proceedings 524(1), 385$388,2000$.

[55] J. H. Marburger: Self-focusing: Theory. Progr. Quant. Electr. 4(Part 1), $35-110,1975$.

[56] F. Merle und P. Raphael: Sharp upper bound on the blow-up rate for the critical nonlinear Schrödinger equation. Geom. Funct. Anal. 13(3), 591-642, 2003.

[57] K. D. Moll, A. L. Gaeta und G. Fibich: Self-Similar Optical Wave Collapse: Observation of the Townes Profile. Phys. Rev. Lett. 90(20), 203902, May 2003.

[58] J. Noack und A. Vogel: Laser-Induced Plasma Formation in Water at Nanosecond to Femtosecond Time Scales: Calculation of Thresholds, Absorption Coefficients, and Energy Density. J. Quant. Electr. 35(8), 1156-1167, 1999.

[59] J.-P. Pérez: Optik. Spektrum Akademischer Verlag, 1996.

[60] M. A. Porras: Diffraction effects in few-cycle optical pulses. Phys. Rev. E 65(2), 026606, 2002.

[61] W. H. Press, S. A. Teukolsky, W. T. Vetterling und B. P. Flannery: Numerical recipes in $C$ : the art of scientific computing. Cambridge University Press, 1992.

[62] B. Rethfeld: Unified Model for the Free-Electron Avalanche in LaserIrradiated Dielectrics. Phys. Rev. Lett. 92(18), 187401, 2004.

[63] B. Rethfeld: Free-electron generation in laser-irradiated dielectrics. Phys. Rev. B 73(3), 035101, 2006.

[64] B. K. Ridley: Quantum processes in semiconductors. Oxford University Press, 1999.

[65] J. E. Rothenberg: Pulse splitting during self-focusing in normally dispersive media. Opt. Lett. 17(8), 583-585, 1992.

[66] C. A. Sacchi: Laser-induced electric breakdown in water. J. Opt. Soc. Am. B 8(2), 337-345, 1991.

[67] T. D. Scarborough, C. Petersen und C. J. Uiterwaal: Measurements of the GVD of water and methanol and laser pulse characterization using direct imaging methods. New J. Phys. 10(10), 103011 (10pp), 2008. 
[68] B. Schäfer und K. Mann: Determination of Beam Parameters and Coherence Properties of Laser Radiation by Use of an Extended HartmannShack Wave-Front Sensor. Appl. Opt. 41(15), 2809-2817, 2002.

[69] Y. R. Shen: Self-focusing: Experimental. Progr. Quant. Electr. 4(Part 1), $1-34,1975$.

[70] Y. R. Shen: The principles of nonlinear optics. Wiley, 2003.

[71] A. E. Siegman: Lasers. University Science Books, Mill Valley, 1986.

[72] O. V. Sinkin, R. Holzlöhner, J. Zweck und C. R. Menyuk: Optimization of the Split-Step Fourier Method in Modeling Optical-Fiber Communications Systems. J. Lightwave Technol. 21(1), 61, 2003.

[73] A. Sommerfeld und H. Bethe: Elektronentheorie der Metalle. Handbuch der Physik 24(2), 333-622, 1933.

[74] C. Sulem und P.-L. Sulem: The Nonlinear Schrödinger Equation: SelfFocusing and Wave Collapse. Springer, New York, 1999.

[75] Q. Sun, H. Jiang, Y. Liu, Z. Wu, H. Yang und Q. Gong: Measurement of the collision time of dense electronic plasma induced by a femtosecond laser in fused silica. Opt. Lett. 30, 320-322, 2005.

[76] R. L. Sutherland: Handbook of Nonlinear Optics. Dekker, 1996.

[77] V. I. Talanov: Focusing of light in cubic media. JETP Lett. (engl.) 11(6), 199-201, 1970.

[78] The International Association for the Properties of Water and Steam: Release on the Refractive Index of Ordinary Water Substance as a Function of Wavelength, Temperature and Pressure. Erlangen, 1997.

[79] The International Association for the Properties of Water and Steam: Revised Release on the IAPWS Industrial Formulation 1997 for the Thermodynamic Properties of Water and Steam. Luzern, 2007.

[80] A. Vogel, J. Noack, G. Hüttman und G. Paltauf: Mechanisms of femtosecond laser nanosurgery of cells and tissues. Appl. Phys. B 81, 1015-1047, 2005.

[81] H. Vogel: Gerthsen Physik. Springer, 1999.

[82] L. T. Vuong, T. D. Grow, A. Ishaaya, A. L. Gaeta, G. W. 't Hooft, E. R. Eliel und G. Fibich: Collapse of Optical Vortices. Phys. Rev. Lett. 96(13), 133901, 2006.

[83] M. I. Weinstein: Nonlinear Schrödinger equations and sharp interpolation estimates. Commun. Math. Phys. 87(4), 567-576, 1983. 


\section{Danksagung}

$\mathrm{Zu}$ guter Letzt möchte ich allen danken, die mich bei der Erstellung dieser Arbeit unterstützt haben. Dabei gilt mein Dank insbesondere ...

... Prof. Dr. Werner Lauterborn für die Möglichkeit, in seiner Arbeitsgruppe mitarbeiten und meine Doktorarbeit schreiben zu können. Für seine Unterstützung möchte ich ihm sehr herzlich danken.

... Prof. Dr. Ulrich Parlitz für die Übernahme des Korreferates und seine aufmunternden und motivierenden Worte zwischendurch.

... Dr. Thomas Kurz, der mich auf das Thema meiner Arbeit gebracht und viele Ideen beigetragen hat.

... meinem langjährigen Zimmernachbarn und guten Freund Dr. Dennis Kröninger. Ihm möchte ich für die tolle Zusammenarbeit und die Bereitstellung seiner experimentellen Daten danken. Mit großer Freude und etwas Wehmut denke ich an die vielen gemeinsamen Stunden im Büro zurück.

... meinem guten Freund und ehemaligen Kollegen Dr. Alexander Ahlborn für das Korrekturlesen dieser Arbeit, seinen fachlichen Rat und seine Unterstützung während und nach unserer gemeinsamen Zeit am Institut.

... den vielen Menschen, die ich in meiner Zeit am Dritten Physikalischen Institut getroffen habe. Ganz besonders möchte ich Dr. Robert Mettin, Gisa Kirschmann-Schröder, meinen Mitstreitern Jörg Dittmar und David Engster sowie meinen Kollegen Till Nowak, Hendrik Söhnholz und Dr. Daniel Schanz danken, die mich durch fachlichen Austausch unterstützt, motiviert und u. a. beim Kickern für Ausgleich gesorgt haben.

... meiner Freundin Inga Schmid für die großartige Unterstützung weit über die Arbeit hinaus. Danke, dass Du immer zu mir hältst!

... meinen Eltern Hannelore und Wolfhard Köhler für das große Verständnis und die Ermutigung zum Schreiben dieser Arbeit sowie ihre große Unterstützung und das Korrekturlesen. Dank gilt auch meiner Schwester Dr. Karola Köhler für ihr Interesse an meiner Arbeit aus der Ferne. 
... meinen Freunden Florian Alpers, Moritz Kirschmann, Dr. Kevin Kröninger, Matthias Lange, Michael Messow und Marc Pauli dafür, dass sie durch nächtliches Zocken, Sport und Spaß immer für die nötige Ablenkung gesorgt haben und in stressigen Zeiten viel Verständnis hatten.

... Lena Schmid, Martina Schmid-Joachim und Hans-Jörg Schmid. Sie haben bis zum Schluß an mich geglaubt und mich immer wieder ermutigt, die Arbeit fertigzustellen. 


\section{Lebenslauf}

Persönliche Daten

Name:

Geburtsdatum:

Geburtsort:

Nationalität:

\section{Schulausbildung}

1985 - 1989:

1989 - 1991:

1991 - 1998:

\section{Studium}

1998 - 2004:

2000:

2004:

\section{Berufliche Tätigkeiten}

2000 - 2002:

$2004-2005:$

2005 - 2008:

seit 2008:
Karsten Köhler

15.02.1979

Göttingen

deutsch

Grundschule Diemarden

Orientierungsstufe Lutherschule in Göttingen

Europaschule Theodor-Heuss-Gymnasium in Göttingen

Physikstudium an der Georg-August-Universität in Göttingen

Vordiplom Physik

Diplom Physik

Studentische Hilfskraft am Institut für Numerische und Angewandte Mathematik der Universität Göttingen

Wissenschaftliche Hilfskraft am Dritten Physikalischen Institut der Universität Göttingen

Wissenschaftlicher Mitarbeiter am Dritten Physikalischen Institut der Universität Göttingen

Selbständige Tätigkeit als Software-Entwickler 